\title{
DYNAMICS OF NONLINEAR WAVE EQUATIONS
}

\author{
CHANGXING MIAO AND JIQIANG ZHENG
}

\begin{abstract}
In this lecture, we will survey the study of dynamics of the nonlinear wave equation in recent years. We refer to some lecture notes including such as C. Kenig 75, 76, C. Kenig and F. Merle 77. J. Shatah and M. Struwe [125], and C. Sogge 131] etc. This lecture was written for LIASFMA School and Workshop on Harmonic Analysis and Wave Equations in Fudan universty.
\end{abstract}

\section{Contents}

1. Introduction

1.1. Behavior of linear solution

1.2. Strichartz estimates

1.3. Finite speed of propagation

1.4. Morawetz estimate

1.5. Linear profile decomposition

2. Main result

2.2. Scattering norm estimate 14

2.3. Threshold of solutions

2.4. Beyond threshold of ground state 17

2.5. Existence of type I blow up solutions 17

2.6. Existence of type II blowup solutions 18

2.7. Description of type II blow up solutions 19

2.8. Bounds on the speed of type II blow-up 20

2.9. Soliton resolution conjecture 21

2.10. Blowup at infinity for the critical wave equation 25

2.11. Two-bubble solutions 25

2.12. Global dynamics away from the ground state 26

2.13. Unconditional uniqueness 26

2.14. Ill-posedness 27

3. Energy-supcritical NLW 27

3.1. Critical norm conjecture 29

3.2. Nonscattering radial solution 30

3.3. Stability in $H^{2} \times H^{1}$ of the ODE blowup profile

3.4. Type II blowup solutions 31

4. Energy-subcritical NLW 33

4.1. Critical norm conjecture 33

4.2. Low regularity 38

4.3. Description of local singularity for type I blow up bubble 40

5. Nonlinear Klein-Gordon equation 40

5.1. Focusing case 42 
6. Proof of defocusing energy-critical NLW 45

7. Proof of sacttering/blowup dichotomy (Theorem 2.5 48

7.1. Local Cauchy problem 49

7.2. Variational estimates 52

7.3. Proof of finite-time blowup

7.4. Concentration-compactness procedure 57

7.5. Zero momentum of critical element

7.6. Rigidity theorem 63

References

\section{INTRODUCTION}

We study the initial-value problem for nonlinear wave equations as following

$$
(\mathrm{NLW}) \quad\left\{\begin{array}{l}
\partial_{t t} u-\Delta u=\mu|u|^{p-1} u, \quad(t, x) \in \mathbb{R} \times \mathbb{R}^{d}, \\
\left(u, \partial_{t} u\right)(0, x)=\left(u_{0}, u_{1}\right)(x),
\end{array}\right.
$$

where $u: \mathbb{R}_{t} \times \mathbb{R}_{x}^{d} \rightarrow \mathbb{R}, \mu= \pm 1$ with $\mu=-1$ known as the defocusing case and $\mu=1$ as the focusing case. Equation (1.1) admits a number of symmetries, explicitly:

- Space-time translation invariance: if $u(t, x)$ solves (1.1), then so does $u\left(t+t_{0}, x+x_{0}\right),\left(t_{0}, x_{0}\right) \in \mathbb{R} \times \mathbb{R}^{d} ;$

- Lorentz transformations invariance: if $u(t, x)$ solves 1.1 , then so does

$$
u\left(\frac{t-\ell \cdot x}{\sqrt{1-|\ell|^{2}}},\left(-\frac{t}{\sqrt{1-|\ell|^{2}}}+\frac{1}{|\ell|^{2}}\left(\frac{1}{\sqrt{1-|\ell|^{2}}}-1\right) \ell \cdot x\right) \ell+x\right)
$$

where $\ell \in \mathbb{R}^{d},|\ell|<1$.

- Scaling invariance: if $u(t, x)$ solves (1.1), then so does $u_{\lambda}(t, x)$ defined by

$$
u_{\lambda}(t, x)=\lambda^{\frac{2}{p-1}} u(\lambda t, \lambda x), \quad \lambda>0 .
$$

This scaling defines a notion of criticality for 1.1). In particular, one can check that the only homogeneous $L_{x}^{2}$-based Sobolev space that is left invariant under 1.3 is $\dot{H}_{x}^{s_{c}}\left(\mathbb{R}^{d}\right)$, where the critical regularity $s_{c}$ is given by $s_{c}:=\frac{d}{2}-\frac{2}{p-1}$.

- For $s_{c}=1$, we call the problem 1.1 energy-critical;

- For $s_{c}<1$, we call the problem (1.1) energy-subcritical;

- For $s_{c}>1$, we call the problem 1.1 energy supercritical.

From the Ehrenfest law or direct computation, the above symmetries induce invariances in the energy space, namely

Energy: $E(u)(t) \triangleq \frac{1}{2} \int_{\mathbb{R}^{d}}\left(|\nabla u|^{2}+\left|\partial_{t} u\right|^{2}\right) d x-\frac{\mu}{p+1} \int_{\mathbb{R}^{d}}|u(t, x)|^{p+1} d x=E\left(u_{0}, u_{1}\right)$,

Momentum: $P(u)(t) \triangleq \int \nabla u u_{t} d x=P\left(u_{0}, u_{1}\right)$.

To begin, we need a few definitions.

Definition 1.1 (classical, weak, strong, strong Strichartz solution). . 
- Classical solution: A function $u$ is a classical solution of (1.1) on a time interval $I$ containing 0 if $u \in C^{2}\left(I \times \mathbb{R}^{d}\right)$ and solves 1.1) in the classical sense.

- Weak solution: A function $u$ is a weak solution of (1.1) if $\left(\partial_{t} u, \nabla_{x} u\right) \in$ $L_{t}^{\infty}\left(\mathbb{R}, L_{x}^{2}\right), u \in L_{t}^{\infty} L_{x}^{p+1}$, and $u$ solves (1.1) in distribution sense, namely

$$
\begin{aligned}
\int_{0}^{+\infty} \int_{\mathbb{R}^{d}} u \square \varphi d x d t & +\int_{0}^{+\infty} \int_{\mathbb{R}^{d}} f(u) \varphi d x d t=-\int_{\mathbb{R}^{d}} u_{0}(x) \partial_{t} \varphi(0, x) d x \\
& +\int_{\mathbb{R}^{d}} u_{1}(x) \varphi(0, x) d x, \quad \forall \varphi \in \mathcal{D}\left(\mathbb{R} \times \mathbb{R}^{d}\right),
\end{aligned}
$$

and the energy inequality

$$
E(u(t)) \leq E(u(0)), \quad \forall t \in \mathbb{R}
$$

holds. Here $f(u)= \pm|u|^{p-1} u$ and $\square=\partial_{t}^{2}-\Delta_{x}$ is the d'Alembertian operator.

- Strong solution: $u: I \times \mathbb{R}^{d} \rightarrow \mathbb{R}$ is a strong solution of (1.1) with data $(f, g) \in H^{s} \times H^{s-1}\left(\mathbb{R}^{d}\right), s \in \mathbb{R}$, if $u$ satisfies the Duhamel formula

$$
\begin{aligned}
u(t, \cdot)= & \cos (t|\nabla|) f+\frac{\sin (t|\nabla|)}{|\nabla|} g \\
& -\mu \int_{0}^{t} \frac{\sin ((t-s)|\nabla|)}{|\nabla|}\left[u(s, \cdot)|u(s, \cdot)|^{p-1}\right] d s
\end{aligned}
$$

for $t$ in time interval $I$ containing 0 .

- Strong Strichartz solution: we say that $u$ is a strong Strichartz solution of (1.1), if $u$ is a strong solution and $u$ belongs to some auxiliary spaces associated with the Strichartz estimate, such as some spatial-time space $L_{t}^{q}\left(I, L_{x}^{r}\left(\mathbb{R}^{d}\right)\right)$.

Remark 1.2. The existence of global weak solutions is proved by Strauss [134.

Definition 1.3 (Well-posedness). We say that the Cauchy Problem (1.1) for (NLW) is locally well-posed (LWP) in $H^{s}$, if for every $\left(u_{0}, u_{1}\right) \in H_{x}^{s} \times H_{x}^{s-1}$

(i) There exist a time $T=T\left(u_{0}, u_{1}\right)>0$ and a distributional solution $u$ : $[-T, T] \times \mathbb{R}^{d} \rightarrow \mathbb{R}$ to 1.1 which is in the space $C^{0}\left([-T, T] ; H_{x}^{s}\right) \cap C^{1}\left([-T, T] ; H_{x}^{s-1}\right)$;

(ii) The solution map $\left(u_{0}, u_{1}\right) \mapsto u$ is uniformly continuous ${ }^{1}$ from $H^{s} \times H^{s-1}$ to $C^{0}\left([-T, T] ; H_{x}^{s}\right) \cap C^{1}\left([-T, T] ; H_{x}^{s-1}\right)$;

(iii) Furthermore, there is an additional space $X$ in which $u$ lies, such that $u$ is the unique solution to the Cauchy problem in $C^{0}\left([-T, T] ; H_{x}^{s}\right) \cap C^{1}\left([-T, T] ; H_{x}^{s-1}\right) \cap$ $X$; and $X \subset L_{t, x, \text { loc }}^{p}$, so $|u|^{p-1} u$ is a well-defined spacetime distribution.

Moreover, if $T=+\infty$, we say that equation (1.1) is globally well-posed (GWP) in $H^{s}$.

\section{Basic mathematical problems in Nonlinear PDEs}

\footnotetext{
${ }^{1}$ Here, we require the solution mapping to be continuous but not necessarily uniformly continuous, as is the case for certain equations, such as Burgers' equation, the Korteweg-de Vries and Benjamin-Ono equations in the periodic case.
} 
- Wellposedness: Existence, uniqueness, continuous dependence on the data, persistence of regularity. The first problem is to understand this locally in time.

- Global behavior: Global existence, or finite time break down(In some norm such as $\|\cdot\|_{H_{x}^{1}\left(\mathbb{R}^{d}\right)}$, becomes unbounded in the finite time)?

- Blow-up dynamics: If the solution breaks down in finite time, can we describe the mechanism by which it dose so? For example, via energy concentration at the tip of a light cone? Usually, symmetries play a crucial role. How about the qualitative description of singularity formation and blowup rate? Solitary wave conjecture, solitary resolution conjecture.

- Scattering theory: If the solutions exist for all $t \in \mathbb{R}$, does it approach a free solution?Critical norm conjecture.

- Special solution: If the solution does not approach a free solution, does it scatter to something else? A stationary nonzero solution, for example? Some physical equations exhibit nonlinear bound states, which represent elementary particles.

- Stability theory: If special solutions exist such as stationary or timeperiodic solutions, are they orbitally stable? are they asymptotically stable?

- Multi-bump solutions: Is it possible to construct solutions which are asymptotically splited into moving "solitons " plus radiation? Lorentz invariance dictates the dynamics of single solitons.

- Resolution into Multi-bumps: Do all solutions decompose in this fashion? Suppose solutions exist for all $t \geq 0$ : Either scatter to a free wave, or the energy collects in "pockets" formed by such "solitons" ? Quantization of energy.

Next, we introduce some basic analysis tools.

\subsection{Behavior of linear solution.}

Lemma 1.4 (Dispersive estimate, Shatah-Struwe [125]). If $d \geq 1,2 \leq q<\infty$, then

$$
\left\|\frac{\sin (t|\nabla|)}{|\nabla|} f\right\|_{L_{x}^{q}\left(\mathbb{R}^{d}\right)} \leq C|t|^{-(d-1)\left(\frac{1}{2}-\frac{1}{q}\right)}\left\||\nabla|^{\frac{d-3}{2}-\frac{d+1}{q}} \nabla f\right\|_{L_{x}^{q^{\prime}}\left(\mathbb{R}^{d}\right)} .
$$

Especially for $d=$ odd number, (1.7) holds for $2 \leq q \leq \infty$.

Lemma 1.5 (Semi-classical dispersive estimate). Let $\psi(r) \in C_{c}^{\infty}(\mathbb{R})$ and $\operatorname{supp} \psi \subset$ $\{r: 1 \leq r \leq 2\}$. Then,

$$
\left\|\psi(h \sqrt{-\Delta}) e^{i t \sqrt{-\Delta}}\right\|_{L_{x}^{1}\left(\mathbb{R}^{d}\right) \rightarrow L_{x}^{\infty}\left(\mathbb{R}^{d}\right)} \leq c h^{-d} \min \left\{1,\left(\frac{h}{t}\right)^{\frac{d-1}{2}}\right\} .
$$

Proof. Denote

$$
I_{h}(t) \triangleq \psi(h \sqrt{-\Delta}) e^{i t \sqrt{-\Delta}} \delta(x)=h^{-d} \int_{\mathbb{R}^{d}} e^{\frac{i t}{h} \Phi(x, t, \xi)} \psi(|\xi|) d \xi,
$$

where $\Phi(x, t, \xi)=\frac{x}{t} \cdot \xi+|\xi|$.

It is easy to see that

$$
\left|I_{h}(t)\right| \lesssim h^{-d}
$$

Hence, it suffices to consider the case $|t|>h$. Without loss of generality, we may assume $t>h$. 
Since $\nabla_{\xi} \Phi=\frac{x}{t}+\frac{\xi}{|\xi|}$, we know that

$$
\left|\nabla_{\xi} \Phi\right| \geq \frac{1}{2}, \text { when }|x| \geq 2 t \text { or }|x| \leq \frac{t}{2} .
$$

In this case, we have by stationary phase argument

$$
\left|I_{h}(t)\right| \lesssim_{k} h^{-d}\left(\frac{t}{h}\right)^{-k}, \quad \forall k \in \mathbb{N} .
$$

If $\frac{1}{2} t \leq|x| \leq 2 t$, then, we get

$$
\begin{aligned}
\left|I_{h}(t)\right| & =h^{-d}\left|\int_{0}^{\infty} e^{\frac{i t}{h} \rho} \rho^{d-1} \psi(\rho) d \rho \int_{\mathbb{S}^{d-1}} e^{i \frac{x}{h} \cdot w \rho} d \sigma(w)\right| \\
& \lesssim h^{-d}\left(\frac{|x|}{h}\right)^{-\frac{d-1}{2}} \lesssim h^{-d}\left(\frac{h}{t}\right)^{\frac{d-1}{2}}
\end{aligned}
$$

where we use the fact

$$
\int_{\mathbb{S}^{d-1}} e^{i \frac{x}{h} \cdot w \rho} d \sigma(w)=2 \pi\left(\frac{|x| \rho}{h}\right)^{-\frac{d-2}{2}} J_{\frac{d-2}{2}}\left(2 \pi \frac{|x| \rho}{h}\right) .
$$

Thus, we obtain 1.8.

Pointwise convergence: The pointwise convergence problem is to ask what the minimal $s$ is to ensure

$$
\lim _{t \rightarrow 0} e^{i t \sqrt{-\Delta}} f(x)=f(x), \quad \text { a.e. } x, \quad \forall f \in H^{s}\left(\mathbb{R}^{d}\right) .
$$

By a standard process of approximation and Fatou's lemma, (1.9) follows from the local maximal inequality

$$
\left\|\sup _{|t|<1}\left|e^{i t \sqrt{-\Delta}} f\right|\right\|_{L^{2}(B(0,1))} \leq C_{s, d}\|f\|_{H^{s}\left(\mathbb{R}^{d}\right)} .
$$

If $s>\frac{d}{2}$, we obtain 1.10 immediately from Sobolev's embedding. It has been known that 1.10 holds for $s>\frac{1}{2}$ in [129, 143] and that this is not true when $s \leq \frac{1}{2}$ is due to B. G. Walther [145].

1.2. Strichartz estimates. To obtain the well-posedness, the key estimate is the Strichartz estimate.

Definition 1.6 (Wave-admissible pair). We say that the exponent pair $(q, r) \in \tilde{\Lambda}$ is wave-admissible, if $2 \leq q, r \leq \infty$ and

$$
\frac{2}{q} \leq(d-1)\left(\frac{1}{2}-\frac{1}{r}\right),(q, r, d) \neq(2, \infty, 3) .
$$

If equality holds in (1.11), we say that $(q, r)$ is sharp wave-admissible, otherwise we say that $(q, r)$ is non-sharp wave-admissible.

Define

$$
\beta(r) \triangleq \frac{d+1}{2}\left(\frac{1}{2}-\frac{1}{r}\right), \delta(r) \triangleq d\left(\frac{1}{2}-\frac{1}{r}\right), \gamma(r) \triangleq(d-1)\left(\frac{1}{2}-\frac{1}{r}\right) .
$$

Theorem 1.7 (Strichartz estimate). Let $d \geq 2$ and $u: I \times \mathbb{R}^{d} \rightarrow \mathbb{R}$ be the solution to $\partial_{t t} u-\Delta u=F$ with initial data $\left(u, \partial_{t} u\right)(0)=(f, g)$. Then, we have

$$
\|u(t, x)\|_{L_{t}^{q_{1}}\left(I, L_{x}^{r_{1}}\left(\mathbb{R}^{d}\right)\right)} \lesssim\|(f, g)\|_{\dot{H}^{s} \times \dot{H}^{s-1}}+\|F\|_{L_{t}^{q_{2}^{\prime}}\left(I, L_{x}^{r_{2}^{\prime}}\right)}
$$


where $\left(q_{1}, r_{1}\right),\left(q_{2}, r_{2}\right) \in \tilde{\Lambda}$ satisfy scaling gap condition

$$
\frac{1}{q_{1}}+\frac{d}{r_{1}}=\frac{d}{2}-s=\frac{1}{q_{2}^{\prime}}+\frac{d}{r_{2}^{\prime}}-2
$$

In level of frequency, we have for $\forall\left(q_{1}, r_{1}\right),\left(q_{2}, r_{2}\right) \in \tilde{\Lambda}$

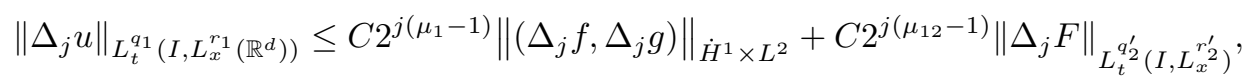
with

$$
\mu_{1}=\delta\left(r_{1}\right)-\frac{1}{q_{1}}, \quad \mu_{12}=\delta\left(r_{1}\right)+\delta\left(r_{2}\right)-\frac{1}{q_{1}}-\frac{1}{q_{2}} .
$$

\subsection{Finite speed of propagation.}

Lemma 1.8 (Huygens principal). (i) Let $d=2 k+1, k \in \mathbb{N}$, then

$$
\frac{\sin (t|\nabla|)}{|\nabla|} f(x)=c_{d}\left(t^{-1} \partial_{t}\right)^{\frac{d-3}{2}}\left(t^{-1} \int_{|y-x|=t} f(y) d \sigma(y)\right),
$$

In particular, when $d=3$, we have

$$
\frac{\sin (t|\nabla|)}{|\nabla|} f(x)=\frac{1}{4 \pi t} \int_{|y-x|=t} f(y) d \sigma(y) .
$$

Let $d=2 k, k \in \mathbb{N}$, then

$$
\frac{\sin (t|\nabla|)}{|\nabla|} f(x)=c_{d}\left(t^{-1} \partial_{t}\right)^{\frac{d-2}{2}}\left(t^{-1} \int_{|y-x| \leq t} f(y) d \sigma(y)\right) .
$$

(ii) (Domain of influence) Let $d=2 k+1, k \in \mathbb{N}$, then

$$
\frac{\sin (t|\nabla|)}{|\nabla|}\left(\chi_{|x| \leq R} f\right)=\chi_{|x|-|t| \mid \leq R} \frac{\sin (t|\nabla|)}{|\nabla|}\left(\chi_{|x| \leq R} f\right) .
$$

(iii) ( Domain of determincy) Let $d=2 k+1, k \in \mathbb{N}$, then

$$
\chi_{|x| \leq R}\left(\frac{\sin (t|\nabla|)}{|\nabla|} f\right)=\chi_{|x| \leq R}\left(\frac{\sin (t|\nabla|)}{|\nabla|} \chi_{|| x|-| t|| \leq R} f\right) .
$$

Proof. (ii) and (iii) are the direct consequence of (1.15).
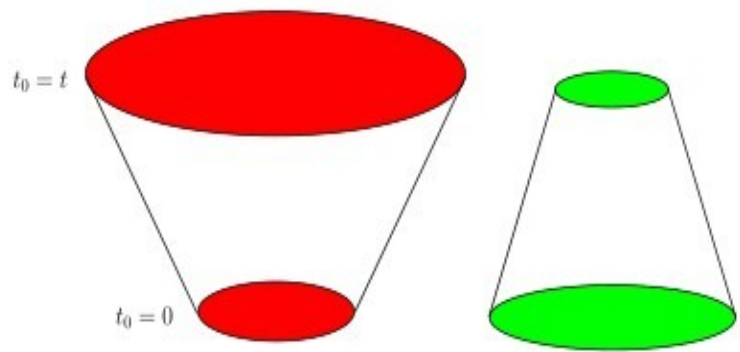

Lemma 1.9 (Finite propagation speed). Let $u: I_{u} \times \mathbb{R}^{d} \rightarrow \mathbb{R}$ and $v: I_{v} \times \mathbb{R}^{d} \rightarrow \mathbb{R}$ solve $\phi_{t t}-\Delta \phi+|\phi|^{p} \phi=0$, with initial data $\left(u_{0}, u_{1}\right),\left(v_{0}, v_{1}\right) \in \dot{H}^{s_{c}} \times \dot{H}^{s_{c}-1}$, respectively, such that for $J \subset \subset I_{u} \cap I_{v}$

$$
\|u\|_{L_{t x}^{p_{c}}\left(J \times \mathbb{R}^{d}\right)}+\|v\|_{L_{t x}^{p_{c}}\left(J \times \mathbb{R}^{d}\right)}<\infty, p_{c}=\frac{d+1}{2} p .
$$


then we have following properties

(1) If initial data satisfies

$$
\left(u_{0}, u_{1}\right)=\left(v_{0}, v_{1}\right), x \in B\left(x_{0}, M\right),
$$

then for any $t \in J$,

$$
u(t, \cdot)=v(t, \cdot), x \in B\left(x_{0}, M-|t|\right), \quad|t| \leq M .
$$

(2) If initial data satisfies

$$
\left(u_{0}, u_{1}\right)=\left(v_{0}, v_{1}\right), x \in B\left(x_{0}, M\right)^{c},
$$

then

$$
u(t, \cdot)=v(t, \cdot), x \in B\left(x_{0}, M+|t|\right)^{c}, \quad \forall t \in J .
$$

where $\left(p_{c}, p_{c}\right) \in \tilde{\Lambda}$ with

$$
\delta\left(p_{c}\right)-\frac{1}{p_{c}}=s_{c} \triangleq \frac{d}{2}-\frac{2}{p} .
$$

\subsection{Morawetz estimate.}

Proposition 1.10 (Morawetz estimate). Let $d \geq 3$, and $u$ be a solution to (1.1) with $\mu=-1$ on a spacetime slab $I \times \mathbb{R}^{d}$. Then

$$
\int_{I} \int_{\mathbb{R}^{d}} \frac{|u|^{p+1}}{|x|} d x d t \leq C(E),
$$

where $E$ is the energy $E\left(u_{0}, u_{1}\right)$.

\subsection{Linear profile decomposition. Let}

$$
S(t)(f, g):=\cos (t|\nabla|) f+\frac{\sin (t|\nabla|)}{|\nabla|} g .
$$

Then the operator $S(t): \dot{H}^{1} \times L_{x}^{2}\left(\mathbb{R}^{d}\right) \rightarrow L_{t, x}^{\frac{2(d+1)}{d-2}}\left(\mathbb{R} \times \mathbb{R}^{d}\right)$ is not compact. This fact shows the linear profile decomposition will be useful in the critical or focusing problem.

Theorem 1.11 (Linear profile decomposition in energy space, [3, 9]). Let $\left(u_{0, n}, u_{1, n}\right)_{n \in \mathbb{N}}$ be a bounded sequence in $\dot{H}^{1} \times L^{2}\left(\mathbb{R}^{d}\right)$ with $d \geq 3$. Then there exists a subsequence of $\left(u_{0, n}, u_{1, n}\right)$ (still denoted by $\left.\left(u_{0, n}, u_{1, n}\right)\right)$, a sequence $\left(V_{0}^{j}, V_{1}^{j}\right)_{j \in \mathbb{N}} \subset \dot{H}^{1} \times L^{2}\left(\mathbb{R}^{d}\right)$ and a sequence of triples $\left(\lambda_{n}^{j}, x_{n}^{j}, t_{n}^{j}\right) \in \mathbb{R}^{+} \times \mathbb{R}^{d} \times \mathbb{R}$ such that for every $j \neq j^{\prime}$,

$$
\frac{\lambda_{n}^{j}}{\lambda_{n}^{j^{\prime}}}+\frac{\lambda_{n}^{j^{\prime}}}{\lambda_{n}^{j}}+\frac{\left|t_{n}^{j}-t_{n}^{j^{\prime}}\right|}{\lambda_{n}^{j}}+\frac{\left|x_{n}^{j}-x_{n}^{j^{\prime}}\right|}{\lambda_{n}^{j}} \underset{n \rightarrow \infty}{\longrightarrow} \infty,
$$

and for every $\ell \geq 1$, if $V^{j}=S(t)\left(V_{0}^{j}, V_{1}^{j}\right)$ and

$$
V_{n}^{j}(t, x) \triangleq \frac{1}{\left(\lambda_{n}^{j}\right)^{\frac{d-2}{2}}} V^{j}\left(\frac{t-t_{n}^{j}}{\lambda_{n}^{j}}, \frac{x-x_{n}^{j}}{\lambda_{n}^{j}}\right),
$$

with

$$
\left\{\begin{array}{l}
u_{0, n}(x)=\sum_{j=1}^{\ell} V_{n}^{j}(0, x)+w_{0, n}^{\ell}(x), \\
u_{1, n}(x)=\sum_{j=1}^{\ell} \partial_{t} V_{n}^{j}(0, x)+w_{1, n}^{\ell}(x),
\end{array}\right.
$$


satisfying

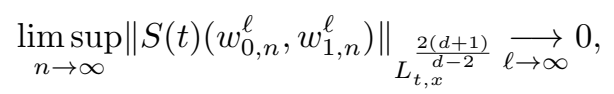

where $S(t)\left(u_{0}, u_{1}\right)$ denotes the solution of the free wave equation with initial data $u(0)=u_{0}$ and $\partial_{t} u(0)=u_{1}$. For every $\ell \geq 1$, we also have

$$
\begin{aligned}
\left\|u_{0, n}\right\|_{\dot{H}^{1}}^{2}+\left\|u_{1, n}\right\|_{L^{2}}^{2}=\sum_{j=1}^{\ell} & \left(\left\|V_{0}^{j}\right\|_{\dot{H}^{1}}^{2}+\left\|V_{1}^{j}\right\|_{L^{2}}^{2}\right)+\left\|w_{0, n}^{\ell}\right\|_{\dot{H}^{1}}^{2} \\
& +\left\|w_{1, n}^{\ell}\right\|_{L^{2}}^{2}+o(1), \quad n \rightarrow \infty,
\end{aligned}
$$

and, for $j \neq k$,

$$
\lim _{n \rightarrow \infty}\left\|V_{n}^{j} V_{n}^{k}\right\|_{L_{t, x}^{\frac{d+1}{d-2}}}=0 .
$$

Applying the profile decomposition to an appropriate sequence, one can obtain the existence of maximizers for Strichartz estimate.

Theorem 1.12 (Existence of maximizers for Strichartz estimate, 9]). Let $d \geq 3$, $(q, r)$ be a wave admissible pair with $q, r \in(2, \infty)$ and satisfying the $\dot{H}^{1}$-scaling condition: $\frac{1}{q}+\frac{d}{r}=\frac{d}{2}-1$. Then there exists a maximizing pair $(\phi, \psi) \in \dot{H}^{1} \times L^{2}\left(\mathbb{R}^{d}\right)$ such that

$$
\|S(t)(\phi, \psi)\|_{L_{t}^{q} L_{x}^{r}\left(\mathbb{R} \times \mathbb{R}^{d}\right)}=C_{q, r}\|(\phi, \psi)\|_{\dot{H}^{1} \times L^{2}\left(\mathbb{R}^{d}\right)}
$$

where

$$
C_{q, r} \triangleq \sup \left\{\|S(t)(\phi, \psi)\|_{L_{t}^{q} L_{x}^{r}}:(\phi, \psi) \in \dot{H}^{1} \times L^{2},\|(\phi, \psi)\|_{\dot{H}^{1} \times L^{2}\left(\mathbb{R}^{d}\right)}=1\right\}
$$

is the sharp constant.

Theorem 1.13 (Linear profile decomposition in $\dot{H}^{s} \times \dot{H}^{s-1}$ with $s \geq 1$, 9]). Let $s \geq 1$ be given and let $\left(u_{0, n}, u_{1, n}\right)_{n \in \mathbb{N}}$ be a bounded sequence in $\dot{H}^{s} \times \dot{H}^{s-1}\left(\mathbb{R}^{d}\right)$ with $d \geq 3$. Then there exists a subsequence of $\left(u_{0, n}, u_{1, n}\right)$ (still denoted $\left(u_{0, n}, u_{1, n}\right)$ ), a sequence $\left(V_{0}^{j}, V_{1}^{j}\right)_{j \in \mathbb{N}} \subset \dot{H}^{s} \times \dot{H}^{s-1}\left(\mathbb{R}^{d}\right)$, and a sequence of triples $\left(\lambda_{n}^{j}, x_{n}^{j}, t_{n}^{j}\right) \in$ $\mathbb{R}^{+} \times \mathbb{R}^{d} \times \mathbb{R}$ such that for every $j \neq j^{\prime}$,

$$
\frac{\lambda_{n}^{j}}{\lambda_{n}^{j^{\prime}}}+\frac{\lambda_{n}^{j^{\prime}}}{\lambda_{n}^{j}}+\frac{\left|t_{n}^{j}-t_{n}^{j^{\prime}}\right|}{\lambda_{n}^{j}}+\frac{\left|x_{n}^{j}-x_{n}^{j^{\prime}}\right|}{\lambda_{n}^{j}} \underset{n \rightarrow \infty}{\longrightarrow} \infty
$$

and for every $\ell \geq 1$, if $V^{j}=S(t)\left(V_{0}^{j}, V_{1}^{j}\right)$ and

$$
\begin{gathered}
V_{n}^{j}(t, x) \triangleq \frac{1}{\left(\lambda_{n}^{j}\right)^{\frac{d-2}{2}}-(s-1)} V^{j}\left(\frac{t-t_{n}^{j}}{\lambda_{n}^{j}}, \frac{x-x_{n}^{j}}{\lambda_{n}^{j}}\right), \\
\left\{\begin{array}{l}
u_{0, n}(x)=\sum_{j=1}^{\ell} V_{n}^{j}(0, x)+w_{0, n}^{\ell}(x), \\
u_{1, n}(x)=\sum_{j=1}^{\ell} \partial_{t} V_{n}^{j}(0, x)+w_{1, n}^{\ell}(x),
\end{array}\right.
\end{gathered}
$$

with

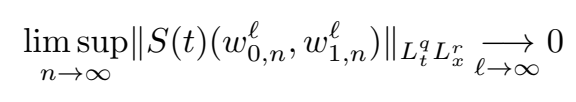


for every $(q, r)$ a wave admissible pair with $q, r \in(2, \infty)$ and satisfying the $\dot{H}^{s}$ scaling condition. For every $\ell \geq 1$, we also have

$$
\begin{aligned}
\left\|u_{0, n}\right\|_{\dot{H}^{s}}^{2}+\left\|u_{1, n}\right\|_{\dot{H}^{s-1}}^{2}=\sum_{j=1}^{\ell} & \left(\left\|V_{0}^{j}\right\|_{\dot{H}^{s}}^{2}+\left\|V_{1}^{j}\right\|_{\dot{H}^{s-1}}^{2}\right)+\left\|w_{0, n}^{\ell}\right\|_{\dot{H}^{s}}^{2} \\
& +\left\|w_{1, n}^{\ell}\right\|_{\dot{H}^{s-1}}^{2}+o(1), \quad n \rightarrow \infty .
\end{aligned}
$$

Theorem 1.14 (Linear profile decomposition in $\dot{H}^{s_{c}}, s_{c}=\frac{1}{2}$, Ramos [118]). Let $\left\{\left(u_{0, n}, u_{1, n}\right)\right\}_{n}$ be a bounded sequence in $\dot{H}^{\frac{1}{2}} \times \dot{H}^{-\frac{1}{2}}\left(\mathbb{R}^{d}\right)$ with $d \geq 2$. Then, there exist a subsequence (still denoted by $\left.\left\{\left(u_{0, n}, u_{1, n}\right)\right\}_{n}\right)$, a sequence $\left(\phi_{0}^{j}, \phi_{1}^{j}\right)_{j \in \mathcal{N}} \subset$ $\dot{H}^{\frac{1}{2}} \times \dot{H}^{-\frac{1}{2}}$ and a family of orthogonal sequences $\left\{\left(r_{j}^{n}, l_{j}^{n}, w_{j}^{n}, x_{j}^{n}, t_{j}^{n}\right)_{n \in \mathbb{N}}\right\}_{j \in \mathbb{N}}$ in $\mathbb{R}^{+} \backslash\{0\} \times[1,+\infty) \times \mathbb{S}^{d-1} \times \mathbb{R}^{d} \times \mathbb{R}$, such that for every $N \geq 1$,

$$
S(t)\left(u_{0, n}, u_{1, n}\right)=\sum_{j=1}^{N} \Gamma_{j}^{n} S(t)\left(\phi_{0}^{j}, \phi_{1}^{j}\right)+S(t)\left(R_{0, n}^{N}, R_{1, n}^{N}\right),
$$

with

$$
\lim _{N \rightarrow \infty} \limsup _{n \rightarrow \infty}\left\|S(t)\left(R_{0, n}^{N}, R_{1, n}^{N}\right)\right\|_{L_{t, x}^{\frac{2(d+1)}{d-1}}}=0
$$

where

$$
\Gamma_{j}^{n} F(x, t):=\left(\frac{r_{j}^{n}}{l_{j}^{n}}\right)^{\frac{d-1}{2}} F\left(\left(T_{w_{j}^{n}}^{l^{n}}\right)^{-1} r_{j}^{n}\left(x-x_{j}^{n}, t-t_{j}^{n}\right)\right),
$$

and $T_{w_{j}^{n}}^{l_{j}^{n}}$ is the rescaled Lorentz transformation defined as

$$
\begin{aligned}
& T_{w}^{2^{j}}(w, 1)=(w, 1), \\
& T_{w}^{2^{j}}(w,-1)=2^{2 j}(2,-1), \\
& T_{w}^{2^{j}}(x, t)=2^{j}(x, t) \quad \text { if }(x, t) \in \mathbb{R}^{d} \text { is orthogonal to }(w, 1) \text { and }(w,-1) .
\end{aligned}
$$

Furthermore, we also have for every $N \geq 1$,

$$
\begin{aligned}
&\left\|u_{0, n}\right\|_{\dot{H}^{\frac{1}{2}}}^{2}+\left\|u_{1, n}\right\|_{\dot{H}^{-\frac{1}{2}}}^{2}=\sum_{j=1}^{\ell}\left(\left\|\phi_{0}^{j}\right\|_{\dot{H}^{\frac{1}{2}}}^{2}+\left\|\phi_{1}^{j}\right\|_{\dot{H}^{-\frac{1}{2}}}^{2}\right)+\left\|R_{0, n}^{\ell}\right\|_{\dot{H}^{\frac{1}{2}}}^{2} \\
&+\left\|R_{1, n}^{\ell}\right\|_{\dot{H}^{-\frac{1}{2}}}^{2}+o(1), \quad n \rightarrow \infty .
\end{aligned}
$$

\section{MAin Result}

History of defocusing energy-subcritical NLW: $\mu=-1, s_{c}<1$.

For $d=3$ and $1<p<p_{c}=5$, in 1961, Jörgen [70] proved the global existence of smooth solution. For higher dimensional case such as $4 \leq d \leq 9$, Brenner-Wahl and Pecher proved the global existence of smooth solution, see [8, 114, 144. Ginibre-Velo proved the global well-posedness of finite enengy solution with initial data $\left(u_{0}, u_{1}\right) \in H^{1} \times L^{2}$ by the compactness argument in [55, 56].

Remark 2.1 (Sketch proof of global existence of smoothing solution). We take $d=3$ for example. For the detailed proof, we refer reader to Chapter 3 in Miao [108. 
Step 1: By the finite propagation speed, we can reduce the above problem to the problem with compact supported data $\left(u_{0}(x), u_{1}(x)\right)$, i.e

$$
\text { supp } u_{0}, \operatorname{supp} u_{1} \subset\{x:|x| \leq R\} .
$$

Step 2: By local theory, if $T^{*} \triangleq \sup I<+\infty$, then $u(t, x) \notin L_{t, x}^{\infty}\left(\left[0, T^{*}\right) \times \mathbb{R}^{3}\right)$. This can be further reduced to

$$
T^{*}<+\infty \Longrightarrow\|u(t, x)\|_{L_{t}^{4}\left(\left[0, T^{*}\right) ; L_{x}^{12}\left(\mathbb{R}^{3}\right)\right)}=+\infty .
$$

Hence, we are reduced to show

$$
u(t, x) \in L_{t}^{4} L_{x}^{12}\left(\left[0, T^{*}\right) \times \mathbb{R}^{3}\right) .
$$

This follows from energy conservation, Strichartz estimate and continuous argument. In fact, assume $T^{*}<+\infty$, then for $0 \leq t_{0}<s<T^{*}$, we have by the Strichartz estimate and Hölder inequality

$$
\begin{aligned}
& \|u\|_{L_{t}^{4} L_{x}^{12}\left(\left[t_{0}, s\right] \times \mathbb{R}^{3}\right)} \lesssim\left\|\left(u_{0}, u_{1}\right)\right\|_{\dot{H}^{1} \times L^{2}}+\left\||u|^{p-1} u\right\|_{L_{t}^{1} L_{x}^{2}\left(\left[t_{0}, s\right] \times \mathbb{R}^{3}\right)} \\
\leq & C E\left(u_{0}, u_{1}\right)^{\frac{1}{2}}+C\|u\|_{L_{t}^{\frac{5}{5-p}} L_{x}^{\frac{12}{7-p}}\left(\left[t_{0}, s\right] \times \mathbb{R}^{3}\right)}\|u\|_{L_{t}^{4} L_{x}^{12}\left(\left[t_{0}, s\right] \times \mathbb{R}^{3}\right)^{-1}} .
\end{aligned}
$$

On the other hand, by the finite propagation speed: $\operatorname{supp} u(t, x) \subset\{x:|x| \leq t+R\}$, we obtain

$$
\begin{aligned}
\|u\|_{L_{t}^{\frac{5}{5-p}} L_{x}^{\frac{12}{7-p}}\left(\left[t_{0}, s\right] \times \mathbb{R}^{3}\right)} & \leq\left(T^{*}-t_{0}\right)^{\frac{5-p}{4}} \sup _{t_{0} \leq t \leq s}\|u(t)\|_{L_{x}^{\frac{12}{7-p}}} \\
& \lesssim\left(T^{*}-t_{0}\right)^{\frac{5-p}{4}}\left(T^{*}+R\right)^{3\left(\frac{7-p}{12}-\frac{1}{p+1}\right)}\|u(t)\|_{L_{x}^{p+1}} \\
& \leq \rho\left(R, T^{*}, E_{0}\right)\left(T^{*}-t_{0}\right)^{\frac{5-p}{4}} .
\end{aligned}
$$

Hence,

$$
\|u\|_{L_{t}^{4} L_{x}^{12}\left(\left[t_{0}, s\right] \times \mathbb{R}^{3}\right)} \leq C E\left(u_{0}, u_{1}\right)^{\frac{1}{2}}+C \rho\left(R, T^{*}, E_{0}\right)\left(T^{*}-t_{0}\right)^{\frac{5-p}{4}}\|u\|_{L_{t}^{4} L_{x}^{12}\left(\left[t_{0}, s\right] \times \mathbb{R}^{3}\right)}^{p-1} .
$$

Note that

$$
\lim _{t_{0} \rightarrow T^{*}} \rho\left(R, T^{*}, E_{0}\right)\left(T^{*}-t_{0}\right)^{\frac{5-p}{4}}=0, \quad \text { for } p<5,
$$

we obtain (2.1) by the continuous argument.

\section{History of energy-critical NLW: $s_{c}=1$.}

For defocusing case $\mu=-1$,

$$
(\mathrm{DNLW})\left\{\begin{array}{l}
u_{t t}-\Delta u+|u|^{\frac{4}{d-2}} u=0,(t, x) \in \mathbb{R} \times \mathbb{R}^{d}, d \geq 3, \\
\left(u, u_{t}\right)(0)=\left(u_{0}, u_{1}\right) .
\end{array}\right.
$$

- For $d=3$ and $p_{c}=5$, in 1988, Rauch [116 proved the global existence of radial smooth solution for (DNLW). In 1990 Grillakis 58 remove radial assumption and proved the global existence of smooth solution for (DNLW) by the classical Morawetz estimate.

- For higher dimensional case such as $3 \leq d \leq 5$, Grillakis [59] proved the global existence of smooth solution for (DNLW) by combining Strichartz estimates with the classical Morawetz estimate.

- Shatah-Struwe 124 proved the global existence of smooth solution for (DNLW) for $d \leq 7$. Moreover, they proved the global existence of finite energy solution in $H^{1} \times L^{2}$ for (DNLW) in 2.3. 
The scattering result is obtained by Bahouri-Gérard [3], and Tao [139]. In particular, Tao in [139] derived a exponential type spacetime bound as follows

$$
\|u\|_{L_{t}^{4} L_{x}^{12}\left(\mathbb{R} \times \mathbb{R}^{3}\right)} \leq C\left(1+E_{0}\right)^{C E_{0}^{105 / 2}}, E_{0}=E\left(u_{0}, u_{1}\right) .
$$

Theorem 2.2 (Defocusing energy-critical NLW). Let $d \geq 3$. Given $\left(u_{0}, u_{1}\right) \in$ $\dot{H}^{1}\left(\mathbb{R}^{d}\right) \times L^{2}\left(\mathbb{R}^{d}\right)$. Then, there is a unique global strong solution $u$ to

$$
\left\{\begin{array}{l}
u_{t t}-\Delta u+|u|^{\frac{4}{d-2}} u=0, \\
\left(u, \partial_{t} u\right)(0)=\left(u_{0}, u_{1}\right) .
\end{array}\right.
$$

Moreover, the solution $u$ obeys the estimate

$$
\int_{\mathbb{R}} \int_{\mathbb{R}^{d}}|u(t, x)|^{\frac{2(d+1)}{d-2}} d x d t \leq C\left(\left\|\left(u_{0}, u_{1}\right)\right\|_{\dot{H}_{x}^{1} \times L_{x}^{2}}\right) .
$$

And so the solution scatters in the sense, there exists $\left(u_{0}^{ \pm}, u_{1}^{ \pm}\right) \in \dot{H}^{1}\left(\mathbb{R}^{d}\right) \times L^{2}\left(\mathbb{R}^{d}\right)$ such that

$$
\lim _{t \rightarrow \pm \infty}\left\|u(t, x)-S(t)\left(u_{0}^{ \pm}, u_{1}^{ \pm}\right)\right\|_{\dot{H}^{1}\left(\mathbb{R}^{d}\right) \times L^{2}\left(\mathbb{R}^{d}\right)}=0,
$$

where

$$
S(t)(f, g)=\cos (t \sqrt{-\Delta}) f+\frac{\sin (t \sqrt{-\Delta})}{\sqrt{-\Delta}} g .
$$

(1) Since there is no pointwise criteria for the critical problem, it seems that GWP and scattering result are simultaneously solved. However, the study history of the $\dot{H}^{1}$-critical wave equation shows us scattering result is later than global well-posedness!

(2) Recently, we give another proof by the concentration-compactness approach to induction on energy, where we show the GWP and scattering simultaneously in [111].

Outline of proof: ( For detail, see Section 6).

By the finite propagation speed of NLW, we can consider the Cauchy problem with compact data.

(1) By ruling out the accumulation of the energy at any time to show the existence of the global smooth solution.

(2) By compactness argument, we can show the global existence and uniqueness of the energy solution.

(3) By FPS and conformal scaling transform, we can prove the scattering result of the energy solution.

Remark 2.3. The scattering theory with radial data is more easier than the general case because the energy accumulation may only occur near the origin in this case. Indeed, by Morawetz estimate

$$
\iint_{\mathbb{R} \times \mathbb{R}^{d}} \frac{|u(t, x)|^{2^{*}}}{|x|} d x d t \leq C(E)<\infty, \quad 2^{*}=\frac{2 d}{d-2}
$$

and radial Sobolev embedding inequality

$$
\left\||x|^{\frac{d}{2}-1} u\right\|_{L_{x}^{\infty}\left(\mathbb{R}^{d}\right)} \leq\|u\|_{\dot{H}^{1}\left(\mathbb{R}^{d}\right)},
$$


we have for $r_{0}=\frac{2(d+1)}{d-2}$

$$
\begin{aligned}
\iint_{\mathbb{R} \times \mathbb{R}^{d}}|u(t, x)|^{r_{0}} d x d t & \leq \iint_{\mathbb{R} \times \mathbb{R}^{d}} \frac{|u(t, x)|^{2^{*}}}{|x|} \cdot|x||u|^{\frac{2}{d-2}} d x d t \\
& \leq\|u\|_{\dot{H}^{1}\left(\mathbb{R}^{d}\right)}^{\frac{2}{d-2}} \iint_{\mathbb{R}^{\prime} \times \mathbb{R}^{d}} \frac{|u|^{2^{*}}}{|x|} d x d t \leq C(E)<\infty,
\end{aligned}
$$

which implies that

$$
\|u\|_{L_{t}^{q_{0}} L_{x}^{r_{0}}\left(\mathbb{R} \times \mathbb{R}^{d}\right)}<\infty, \quad q_{0}=r_{0}=\frac{2(d+1)}{d-2}
$$

with

$$
\frac{2}{q_{0}} \leq \gamma\left(r_{0}\right), \quad \delta\left(r_{0}\right)-\frac{1}{q_{0}}=1, \quad \frac{\delta\left(r_{0}\right)}{d}=\frac{\gamma\left(r_{0}\right)}{d-1}=\frac{1}{2}-\frac{1}{r_{0}} .
$$

We refer the reader to the proof of the nonradial case in Section 6

The dynamics of the focusing energy-critical equation

$$
(\text { FNLW })\left\{\begin{array}{l}
\partial_{t t} u-\Delta u=|u|^{\frac{4}{d-2}} u, \quad(t, x) \in \mathbb{R} \times \mathbb{R}^{d}, \\
\left(u, \partial_{t} u\right)(0, x)=\left(u_{0}, u_{1}\right)(x),
\end{array}\right.
$$

is much richer: small data solutions are global and scatter, however blow-up in finite time may occur [91, where Levine showed that if

$$
\left(u_{0}, u_{1}\right) \in \dot{H}^{1} \times L^{2}, \quad u_{0} \in L^{2}, E\left(u_{0}, u_{1}\right)<0,
$$

there is always break-down in finite time. Recently, Krieger-Schlag-Tataru [88] have constructed explicit radial examples which break down in finite time. On the other hand, it is well-known that there exist solutions with compactly supported smooth initial data which blow up in finite time. This is most easily seen by observing that

$$
u(t, x)=\left(\frac{d(d-2)}{4}\right)^{\frac{d-2}{4}} /(1-t)^{\frac{d-2}{2}}
$$

is an explicit solution which, by finite speed of propagation, can be used to construct a blowup solution of the aforementioned type. This kind of breakdown is referred to as ODE blowup and it is conjectured to comprise the "generic" blowup scenario [5].

Remark 2.4. About the finite blow up solution: Let $Q$ satisfy

$$
-\Delta Q+Q=|Q|^{\frac{4}{d}} Q .
$$

For the mass-critical focusing Schrödinger equation $i \partial_{t} u+\Delta u+|u|^{\frac{4}{d}} u=0$, the pseudo-conformal transformation applied to the stationary solution $e^{i t} Q$ yields an explicit blowup solution

$$
S(t, x)=\frac{1}{|t|^{d / 2}} Q\left(\frac{x}{t}\right) e^{-i \frac{|x|^{2}}{4 t}+\frac{i}{t}}, \quad\|S(t)\|_{L^{2}}=\|Q\|_{L^{2}}
$$

which scatters as $t \rightarrow-\infty$ since

$$
\|S(t, x)\|_{L_{t, x}}^{\frac{2(d+2)}{d}}\left(\begin{array}{l}
\frac{2(d+2)}{d} \\
\left((-\infty,-1) \times \mathbb{R}^{d}\right)
\end{array}=\int_{-\infty}^{-1}|t|^{-2} d t\|Q\|_{L_{x}^{\frac{2(d+2)}{d}}}^{\frac{\frac{2(d+2)}{d}}{d}}<+\infty,\right.
$$


and blows up at $T=0$ at the speed

$$
\|\nabla S(t)\|_{L^{2}} \sim \frac{1}{|t|} .
$$

An essential feature of 2.10 is compact up to the symmetries of the flow, which shows that all the mass goes into the singularity formation

$$
|S(t)|^{2} \rightarrow\|Q\|_{L^{2}}^{2} \delta_{x=0} \text { as } t \rightarrow 0 .
$$

Non-scattering global solutions also exist. Examples of such solutions are the so-called solitary waves and are given by solutions of the elliptic equation:

$$
-\Delta W=|W|^{\frac{4}{d-2}} W, \quad W \in \dot{H}^{1},
$$

(see 25] for the existence of such solutions),

$$
W(x) \triangleq \frac{1}{\left(1+\frac{|x|^{2}}{d(d-2)}\right)^{\frac{d-2}{2}}} \in \dot{H}^{1},
$$

but in $L^{2}$ only if $d \geq 5$. The works of Aubin and Talenti [1, 141, give the following elliptic characterization of $W(x)$

$$
\begin{aligned}
\|u\|_{L^{2^{*}}} & \leq C_{d}\|u\|_{\dot{H}^{1}}, \quad \forall u \in \dot{H}^{1} ; \\
\|u\|_{L^{2 *}} & =C_{d}\|u\|_{\dot{H}^{1}} \Rightarrow \exists \lambda_{0}, x_{0}, \theta_{0} \quad u(x)=e^{i \theta_{0}} \lambda^{\frac{d-2}{2}} W\left(\lambda_{0}\left(x+x_{0}\right)\right)
\end{aligned}
$$

where $C_{d}$ is the best Sobolev constant in dimension $d$.

\subsection{Below threshold of ground state.}

Theorem 2.5 (Scattering/Blowup dichotomy, Kenig-Merle[77]). Assume $3 \leq d \leq$ $5,\left(u_{0}, u_{1}\right) \in \dot{H}^{1} \times L^{2}$ with

$$
E\left(u_{0}, u_{1}\right)<E(W, 0)
$$

Then

(1) If $\left\|u_{0}\right\|_{\dot{H}^{1}}<\|W\|_{\dot{H}^{1}}$, then the solution $u$ of 2.9 is global and scatters.

(2) If $\left\|u_{0}\right\|_{\dot{H}^{1}}>\|W\|_{\dot{H}^{1}}$, then the solutions $u$ of 2.9 blows up in finite time in both directions.

Remark 2.6. (i) The condition $\left\|u_{0}\right\|_{\dot{H}^{1}}=\|W\|_{\dot{H}^{1}}$ is not compatible with $E\left(u_{0}, u_{1}\right)<$ $E(W, 0)$.

(ii) Bulut et.al [14] extended the above result to higher dimensions.

(iii) Sketch proof of scattering part: We proceed "concentration compactness" and profile decomposition developed by Bahouri- Gérard [3] for $d=3$ (see also Bulut $[9]$ for $d \geq 4$ ). Thus, arguing by contradiction, we find a number $E_{c}$, with $0<\eta_{0} \leq E_{c}<E(W, 0)$ with the property that if

$$
E\left(u_{0}, u_{1}\right)<E_{c}, \quad\left\|\nabla u_{0}\right\|_{2}<\|\nabla W\|_{2},
$$

then $\|u\|_{S(I)}<\infty$. And $E_{c}$ is optimal with this property. We will see that this leads to a contradiction.

Step 1: The existence of critical element with compactness. There exists

$$
\left(u_{0, c}, u_{1, c}\right) \in \dot{H}^{1} \times L^{2},\left\|\nabla u_{0, c}\right\|_{2}<\|\nabla W\|_{2}, E\left(u_{0, c}, u_{1, c}\right)=E_{c},
$$


such that for the corresponding solution $u_{c}(t)$, we have

$$
\left\|u_{c}\right\|_{L_{t, x}^{\frac{2(d+1)}{d-2}}\left(I_{+} \times \mathbb{R}^{d}\right)}=+\infty, I_{+}=I \cap[0,+\infty) .
$$

Moreover, there exist $x(t), t \in I_{+}, \lambda(t) \in \mathbb{R}^{+}, t \in I_{+}$, such that

$$
K=\left\{\vec{v}(x, t)=\left(\frac{1}{\lambda(t)^{\frac{d-2}{2}}} u\left(\frac{x-x(t)}{\lambda(t)}, t\right), \frac{1}{\lambda(t)^{\frac{d}{2}}} \partial_{t} u\left(\frac{x-x(t)}{\lambda(t)}, t\right)\right), t \in I_{+}\right\}
$$

has compact closure in $\dot{H}^{1} \times L^{2}$.

Step 2: Rigidity theorem. $T_{+}<\infty$ is impossible, and if $T_{+}=+\infty$, then $u \equiv 0$.

Please refer to Section 7 for complete proof .

Remark 2.7. (i) Kenig-Merle in [77 claimed: Assume that $u$ is a radial solution or $d \in\{3,4\}$ such that

$$
\sup _{t \in\left[0, T_{+}\right)}\left[\|\nabla u\|_{2}^{2}+\left\|\partial_{t} u\right\|_{2}^{2}\right]<\|\nabla W\|_{2}^{2},
$$

then $T_{+}=+\infty$ and $u$ is scatter forward in time. But when $d=5$, the condition 2.16 does not suffice to ensure the solution of (FNLW) scatter forward in time. Please refer the sharp condition in (ii).

(ii) In [38, Duyckaerts-Kenig-Merle prove that: assume $d \in\{3,4,5\}$. Let $u$ be a solution of (FNLW) which satisfies

$$
\limsup _{t \rightarrow T_{+}(u)}\left[\|\nabla u(t)\|_{L^{2}}^{2}+\frac{d-2}{2}\left\|\partial_{t} u(t)\right\|_{L^{2}}^{2}\right]<\|\nabla W\|_{L^{2}}^{2} .
$$

Then $T_{+}(u)=+\infty$ and $u$ scatters forward in time. If $u$ is radial, 2.17) can be replaced by the following bound

$$
\limsup _{t \rightarrow T_{+}(u)}\|\nabla u(t)\|_{L^{2}}^{2}<\|\nabla W\|_{L^{2}}^{2} .
$$

2.2. Scattering norm estimate. Consider

$$
(\mathrm{FNLW})\left\{\begin{array}{l}
\partial_{t t} u-\Delta u=|u|^{\frac{4}{d-2}} u, \quad(t, x) \in \mathbb{R} \times \mathbb{R}^{d}, \\
\left(u, \partial_{t} u\right)(0, x)=\left(u_{0}, u_{1}\right)(x)
\end{array}\right.
$$

where $u: \mathbb{R}_{t} \times \mathbb{R}_{x}^{d} \rightarrow \mathbb{R}$.

In Theorem 2.5. Kenig-Merle [77] have described the dynamics of (2.19) below the energy threshold $E(W, 0)$. Theorem 2.5 implies that for $\varepsilon>0$ the following supremum is finite:

$$
\mathcal{I}_{\varepsilon}=\sup _{u \in F_{\varepsilon}} \int_{\mathbb{R} \times \mathbb{R}^{d}}|u(t, x)|^{\frac{2(d+1)}{d-2}} d t d x=\sup _{u \in F_{\varepsilon}}\|u\|_{S(\mathbb{R})}^{\frac{2(d+1)}{d-2}}<\infty
$$

where

$$
\begin{array}{r}
F_{\varepsilon} \triangleq\left\{u: \text { solution of } 2.19 \text { such that } E\left(u_{0}, u_{1}\right) \leq E(W, 0)-\varepsilon^{2}\right. \\
\text { and } \left.\int\left|\nabla u_{0}\right|^{2}<\int|\nabla W|^{2}\right\} .
\end{array}
$$

Furthermore, the existence of the non-scattering solution $W$ at the energy threshold shows that

$$
\lim _{\varepsilon \rightarrow 0^{+}} \mathcal{I}_{\varepsilon}=+\infty
$$


Consider the negative eigenvalue $-\omega^{2}(\omega>0)$ of the linearized operator associated to 2.19 around $W$ :

$$
-\omega^{2}=\inf _{\substack{u \in H^{1},\|u\|_{2}=1}}\left[\int_{\mathbb{R}^{d}}|\nabla u|^{2} d x-\frac{d+2}{d-2} \int_{\mathbb{R}^{d}} W^{\frac{4}{d-2}}|u|^{2} d x\right] .
$$

Then

Theorem 2.8 (Scattering norm estimate, Duyckaerts-Merle [45]). Let $d \in\{3,4,5\}$. Then, we have

$$
\lim _{\varepsilon \rightarrow 0^{+}} \frac{\mathcal{I}_{\varepsilon}}{|\log \varepsilon|}=\frac{2}{\omega} \int_{\mathbb{R}^{d}} W^{\frac{2(d+1)}{d-2}} d x .
$$

Remark 2.9. (i) It would be interesting to get an explicit value of the limit

$$
\frac{2}{\omega} \int_{\mathbb{R}^{d}} W^{\frac{2(d+1)}{d-2}} d x
$$

A straightforward computation gives:

$$
\begin{array}{ll}
\int_{\mathbb{R}^{d}} W^{\frac{2(d+1)}{d-2}} d x=\frac{(d(d-2))^{\frac{d}{2}}}{2^{2 d+1}} \times \frac{d !}{\left(\left(\frac{d}{2}\right) !\right)^{2}} \times \pi & \text { if } d \text { is even, } \\
\int_{\mathbb{R}^{d}} W^{\frac{2(d+1)}{d-2}} d x=\frac{(d(d-2))^{\frac{d}{2}}}{2} \times \frac{\left(\frac{d-1}{2}\right) !}{d !} & \text { if } d \text { is odd. }
\end{array}
$$

However we do not know any explicit expression of $\omega$.

(ii) The proofs rely on the compactness argument of Kenig-Merle, on a classification result at the energy level $E(W, 0)$, and on the analysis of the linearized equation around $W$.

(iii) It seems to be possible to extend the above result to higher dimensions $d \geq 6$. The authors in [45] also obtain the similar result for NLS, how about the Klein-Gordon equation? and $\varepsilon \rightarrow 0-$ ?

Proposition 2.10. Let $u_{n}$ be a family of solutions of (FNLW) such that

$$
E\left(u_{n}(0), \partial_{t} u_{n}(0)\right)<E(W, 0), \quad\left\|\nabla u_{n}(0)\right\|_{2}<\|\nabla W\|_{2} .
$$

and $\lim _{n \rightarrow+\infty}\left\|u_{n}\right\|_{S(\mathbb{R})}=+\infty$. Let $\left(t_{n}\right)_{n}$ be a time sequence.

(1) Assume that

$$
\lim _{n \rightarrow+\infty}\left\|u_{n}\right\|_{S\left(-\infty, t_{n}\right)}=\lim _{n \rightarrow+\infty}\left\|u_{n}\right\|_{S\left(t_{n},+\infty\right)}=+\infty .
$$

Then, up to the extraction of a subsequence there exist $\iota_{0} \in\{-1,+1\}$ and sequences of parameters $x_{n} \in \mathbb{R}^{d}, \lambda_{n}>0$ such that

$$
\lim _{n \rightarrow+\infty}\left\|\frac{\iota_{0}}{\lambda_{n}^{d / 2}} \nabla u_{n}\left(t_{n}, \frac{-x_{n}}{\lambda_{n}}\right)-\nabla W\right\|_{2}+\left\|\frac{\partial u_{n}}{\partial t}\left(t_{n}\right)\right\|_{2}=0 .
$$

(2) Assume that there exists $C_{0} \in(0,+\infty)$ such that

$$
\lim _{n \rightarrow+\infty}\left\|u_{n}\right\|_{S\left(-\infty, t_{n}\right)}=+\infty \text { and } \lim _{n \rightarrow+\infty}\left\|u_{n}\right\|_{S\left(t_{n},+\infty\right)}=C_{0} .
$$


Then, up to the extraction of a subsequence there exist $t_{0} \in \mathbb{R}, \iota_{0}, \iota_{1} \in$ $\{-1,+1\}$, and sequences of parameters $x_{n} \in \mathbb{R}^{d}, \lambda_{n}>0$ such that

$$
\begin{aligned}
\lim _{n \rightarrow+\infty} \| \frac{\iota_{0}}{\lambda_{n}^{d / 2}} \nabla & u_{n}\left(t_{n}, \frac{\cdot-x_{n}}{\lambda_{n}}\right)-\nabla W^{-}\left(t_{0}\right) \|_{2} \\
& +\left\|\frac{\iota_{1}}{\lambda_{n}^{d / 2}} \frac{\partial u_{n}}{\partial t}\left(t_{n}, \frac{\cdot-x_{n}}{\lambda_{n}}\right)-\frac{\partial W^{-}}{\partial t}\left(t_{0}\right)\right\|_{2}=0
\end{aligned}
$$

where $W^{-}$is as in Theorem 2.11.

2.3. Threshold of solutions. In this subsection, we consider $E\left(u_{0}, u_{1}\right)=E(W, 0)$.

Theorem 2.11 (Connecting orbits, Duyckaerts-Merle 43 for $3 \leq d \leq 5$, Li-Zhang 93 for $d \geq 6)$. Let $d \geq 3$. There exist radial solutions $W^{-}$and $W^{+}$of $(2.9)$, with initial conditions $\left(W_{0}^{ \pm}, W_{1}^{ \pm}\right) \in \dot{H}^{1} \times L^{2}$ such that

$$
\begin{aligned}
& E(W, 0)=E\left(W_{0}^{+}, W_{1}^{+}\right)=E\left(W_{0}^{-}, W_{1}^{-}\right), \\
& T_{+}\left(W^{-}\right)=T_{+}\left(W^{+}\right)=+\infty \text { and } \lim _{t \rightarrow+\infty} W^{ \pm}(t)=W \text { in } \dot{H}^{1}, \\
& \left\|\nabla W^{-}\right\|_{2}<\|\nabla W\|_{2}, T_{-}\left(W^{-}\right)=+\infty,\left\|W^{-}\right\|_{L^{\frac{2(d+1)}{d-2}}}\left((-\infty, 0) \times \mathbb{R}^{d}\right) \\
& \left\|\nabla W^{+}\right\|_{2}>\|\nabla W\|_{2}, \quad T_{-}\left(W^{+}\right)<+\infty .
\end{aligned}
$$

Remark 2.12. (i) The above construction gives a precise asymptotic development of $W^{ \pm}$near $t=+\infty$. Indeed there exists an eigenvalue $e_{0}>0$ of the linearized operator near $W$, such that, if $\mathcal{Y} \in \mathcal{S}$ is the corresponding eigenfunction with the appropriate normalization,

$$
\left\|\nabla\left(W^{ \pm}(t)-W \pm e^{-e_{0} t} \mathcal{Y}\right)\right\|_{L^{2}}+\left\|\partial_{t}\left(W^{ \pm}(t)-W \pm e^{-e_{0} t} \mathcal{Y}\right)\right\|_{L^{2}} \leq C e^{-2 e_{0} t}
$$

(ii) Similar solutions were constructed for NLS in 44, 92. However, in the NLS case, it is not able to prove that $T_{-}\left(W^{+}\right)<\infty$ except in the case $d \geq 5$. We see this fact, in particular in the case $d=3$, as a nontrivial result. Note that $W^{+}$is not in $L^{2}$ except for $d \geq 5$, so that case (3) of Theorem 2.13 below does not apply.

The next result is that $W, W^{-}$and $W^{+}$are, up to the symmetry of the equation, the only examples of new behavior at the critical level.

Theorem 2.13 (Dynamical classification at the critical level [43, [93]). Let $d \geq 3$. Let $\left(u_{0}, u_{1}\right) \in \dot{H}^{1} \times L^{2}$ such that

$$
E\left(u_{0}, u_{1}\right)=E(W, 0)=\frac{1}{d C_{d}^{d}} .
$$

Let $u$ be the solution of (2.9) with initial conditions $\left(u_{0}, u_{1}\right)$ and I its maximal interval of definition. Then the following holds:

(1) If $\left\|\nabla u_{0}\right\|_{2}^{2}<\|\nabla W\|_{2}^{2}=1 / C_{d}^{d}$, then $I=\mathbb{R}$. Furthermore, either $u=W^{-}$ up to the symmetries of the equation, or

$$
\|u\|_{L_{t, x}^{\frac{2(d+1)}{d-2}}\left(\mathbb{R} \times \mathbb{R}^{d}\right)}<\infty .
$$

(2) If $\left\|\nabla u_{0}\right\|_{2}^{2}=\|\nabla W\|_{2}^{2}$, then $u=W$ up to the symmetries of the equation.

(3) If $\left\|\nabla u_{0}\right\|_{2}^{2}>\|\nabla W\|_{2}^{2}$ and $u_{0} \in L^{2}$, then either $u=W^{+}$up to the symmetries of the equation, or I is finite. 
In the above theorem, by $u$ equals $v$ up to the symmetry of the equation, we mean that there exists $t_{0} \in \mathbb{R}, x_{0} \in \mathbb{R}^{N}, \lambda_{0}>0, \iota_{0}, \iota_{1} \in\{-1,+1\}$ such that

$$
u(t, x)=\frac{\iota_{0}}{\lambda_{0}^{(d-2) / 2}} v\left(\frac{t_{0}+\iota_{1} t}{\lambda_{0}}, \frac{x+x_{0}}{\lambda_{0}}\right) .
$$

Remark 2.14. (i) Case $(2)$ is a direct consequence of the variational characterization of $W$ given by $(2.12)$. Furthermore, using assumption 2.26 , it shows by continuity of $u$ in $\dot{H}^{1}$ and the conservation of energy that the assumptions

$$
\left\|\nabla u\left(t_{0}\right)\right\|_{2}^{2}<\|\nabla W\|_{2}^{2}, \quad \text { or }\left\|\nabla u\left(t_{0}\right)\right\|_{2}^{2}>\|\nabla W\|_{2}^{2}
$$

do not depend on the choice of the initial time $t_{0}$. Of course, this dichotomy does not persist when $E\left(u_{0}, u_{1}\right)>E(W, 0)$.

(ii) Theorem 2.13 is also the analoguous, for the wave equation, of Theorem 2 of 44 for NLS, but without any radial assumption. The nonradial situation carries various problems partially solved in [77, the major difficulty being a sharp control in time of the space localization of the energy.

(iii) In dimension $d=3$ or $d=4, W^{+}$is not in $L^{2}$. It is a delicate problem to get rid of the assumption $u_{0} \in L^{2}$.

(iv) The main issue in high dimensions $(d \geq 6)$ is the non-Lipschitz continuity of the nonlinearity which they get around by making full use of the decay property of $W$.

2.4. Beyond threshold of ground state. Next, we consider $E\left(u_{0}, u_{1}\right)>E(W, 0)$. First, we introduce the definition of two type blow up solutions.

Definition 2.15 (Type I blowup solutions). Let $u:\left[0, T_{+}\right) \times \mathbb{R}^{d} \rightarrow \mathbb{R}$ be the maximal life-span solution of (FNLW) satisfying

$$
T_{+}<+\infty, \sup _{t \in\left[0, T_{+}\right)}\left[\|\nabla u\|_{2}^{2}+\left\|\partial_{t} u\right\|_{2}^{2}\right]=+\infty
$$

then we call $u(t, x)$ to be Type I-blowup solution for (FNLW)(unbounded solutions blowing up in finite time). We call it blows up in infinity if

$$
T_{+}=+\infty, \sup _{t \in\left[0, T_{+}\right)}\left[\|\nabla u\|_{2}^{2}+\left\|\partial_{t} u\right\|_{2}^{2}\right]=+\infty \text {. }
$$

Definition 2.16 (Type II blowup solutions). Let $u:\left[0, T_{+}\right) \times \mathbb{R}^{d} \rightarrow \mathbb{R}$ be the maximal life-span solution of (FNLW) with

$$
T_{+}<+\infty, \quad \sup _{t \in\left[0, T_{+}\right)}\left[\|\nabla u\|_{2}^{2}+\left\|\partial_{t} u\right\|_{2}^{2}\right]<+\infty
$$

then we call $u(t, x)$ to be Type II-blowup solution for (FNLW) (bounded solutions blowing up in finite time).

2.5. Existence of type I blow up solutions. We consider the initial value problem for the focusing nonlinear wave equation

$$
\left\{\begin{array}{l}
\partial_{t}^{2} u-\Delta u=|u|^{p-1} u, \quad(t, x) \in I \times \mathbb{R}^{d} \\
\left.u\right|_{t=0}=u_{0},\left.\quad \partial_{t} u\right|_{t=0}=u_{1}, \quad x \in \mathbb{R}^{d}
\end{array}\right.
$$


for $d=2 k+1$ with $k \geq 2$ and $I$ an interval with $0 \in I$. 2.30 is conformally invariant for $p=\frac{d+3}{d-1}$ and we restrict ourselves to the superconformal case

$$
p>\frac{d+3}{d-1} .
$$

Explicit examples for singularity formation can be obtained by considering the so called ODE blowup solution

$$
u_{T}(t, x)=c_{p}(T-t)^{-\frac{2}{p-1}}, \quad c_{p}:=\left[\frac{2(p+1)}{(p-1)^{2}}\right]^{\frac{1}{p-1}}
$$

which is independent of the space dimension and solves the ordinary differential equation $u_{t t}=|u|^{p-1} u$ for $p>1$. By finite speed of propagation one can use $u_{T}$ to construct compactly supported smooth initial data such that the solution blows up as $t \rightarrow T$.

Theorem 2.17 (Existence of type I blow up solution, 35). For $d=2 k+1, k \in \mathbb{N}$ with $k \geq 2$, fix $p>\frac{d+3}{d-1}$ and $T_{0}>0$. There are constants $M, \delta>0$ such that if $u_{0}, u_{1}$ are radial functions with

$$
\left\|\left(u_{0}, u_{1}\right)-u_{T_{0}}[0]\right\|_{H^{\frac{d+1}{2}} \times H^{\frac{d-1}{2}}\left(B_{\left.T_{0}+\delta\right)}\right.}<\frac{\delta}{M},
$$

where $B_{T_{0}+\delta} \triangleq\left\{x:|x| \leq T_{0}+\delta\right\}$. Then

(1) The blowup time at the origin $T:=T_{\left(u_{0}, u_{1}\right)}$ is contained in the interval $\left[T_{0}-\delta, T_{0}+\delta\right]$

(2) The solution $u: \Gamma_{T}\left(\mathbb{R}^{d}\right) \rightarrow \mathbb{R}$ satisfies

$$
\begin{aligned}
& \left\{\begin{array}{l}
(T-t)^{\frac{2}{p-1}}\left\|u(t, \cdot)-u_{T}(t, \cdot)\right\|_{L^{\infty}\left(B_{T-t}\right)} \lesssim(T-t)^{\mu_{p}} \\
(T-t)^{-s_{p}}\left\|u(t, \cdot)-u_{T}(t, \cdot)\right\|_{L^{2}\left(B_{T-t}^{d}\right)} \lesssim(T-t)^{\mu_{p}} \\
(T-t)^{-s_{p}+1}\left\|u[t]-u_{T}[t]\right\|_{\dot{H}^{1} \times L^{2}\left(B_{T-t}\right)} \lesssim(T-t)^{\mu_{p}}
\end{array}\right. \\
& \text { for } s_{p}=\frac{d}{2}-\frac{2}{p-1}, \mu_{p}=\min \left\{\frac{2}{p-1}, 1\right\}-\varepsilon \text { and } \varepsilon>0 \text { small. Furthermore, for } \\
& j=2, \ldots, \frac{d+1}{2}, \\
& \quad(T-t)^{-s_{p}+j}\|u[t]\|_{\dot{H}^{j} \times \dot{H}^{j-1}\left(B_{T-t}\right)} \lesssim(T-t)^{\mu_{p}} .
\end{aligned}
$$

where

$$
\Gamma_{T}\left(\mathbb{R}^{d}\right) \triangleq\left\{(t, r): t \in\left[T_{0}-\delta, T_{0}+\delta\right], r=|x| \in(0, T-t)\right\} .
$$

2.6. Existence of type II blowup solutions. Examples of radial type II blowup solutions of (FNLW) were constructed in space dimension $d=3$ by Krieger, Schlag and Tataru [88, see Theorem 2.18 below.

Theorem 2.18 (Existence of type II blowup solution in $\mathbb{R}^{3}$, 88]). Let $\nu>\frac{1}{2}$ and $\delta>0$. Then there exists an energy solution $u$ of (FNLW) which blows up precisely at $r=t=0$ and which has the following property: in the cone $|x|=r \leq t$ and for small times the solution has the form,

$$
u(x, t)=\lambda^{\frac{1}{2}}(t) W(\lambda(t) r)+\eta(x, t), \quad \lambda(t)=t^{-1-\nu},
$$

where $E_{\mathrm{loc}}(\eta(\cdot, t)) \rightarrow 0$ as $t \rightarrow 0$ and outside the cone $u(x, t)$ satisfies

$$
\int_{[|x| \geq t]}\left[|\nabla u(x, t)|^{2}+\left|u_{t}(x, t)\right|^{2}+|u(x, t)|^{6}\right] d x<\delta
$$


for all sufficiently small $t>0$. In particular, the energy of these blow-up solutions can be chosen arbitrarily close to $E(W, 0)$, i.e., the energy of the stationary solution.

Remark 2.19. (i) Lately, Krieger and Schlag [87 improved this to $\nu>0$

(ii) If $\nu>1$, then the solutions from Theorem 2.18 belong to $L^{\infty}\left(\mathbb{R}^{3}\right)$ for all $t>0$ and blow up at the rate

$$
\|u(\cdot, t)\|_{\infty} \asymp t^{-(1+\nu) / 2}, \text { as } t \rightarrow 0 .
$$

In dimension five, denote $X^{s}:=\dot{H}^{s+1} \cap \dot{H}^{1}$.

Theorem 2.20 (Existence of smooth type II blowup solutions in $d=5$, 67]). Let $\left(u_{0}^{*}, u_{1}^{*}\right) \in X^{4} \times H^{4}$ be any pair of radial functions with $u_{0}^{*}(0)>0$. Let $\left(u^{*}(t), \partial_{t} u^{*}(t)\right)$ be the solution of (FNLW) with the initial data $\left(u^{*}(0), \partial_{t} u^{*}(0)\right)=$ $\left(u_{0}^{*}, u_{1}^{*}\right)$. There exists a solution $\left(u, \partial_{t} u\right)$ of (FNLW) defined on a time interval $\left(0, T_{0}\right)$ and $a C^{1}$ function $\lambda(t):\left(0, T_{0}\right) \rightarrow(0,+\infty)$ such that

$$
\left\{\begin{array}{l}
\left\|\left(u(t)-W_{\lambda(t)}-u^{*}(t), \partial_{t} u(t)+\lambda_{t}(t)(\Lambda W)_{\lambda(t)}-\partial_{t} u^{*}(t)\right)\right\|_{\dot{H}^{1} \times L^{2}}=O\left(t^{9 / 2}\right) ; \\
\lambda(t)=\left(\frac{32}{315 \pi}\right)^{2}\left(u^{*}(0,0)\right)^{2} t^{4}+o\left(t^{4}\right), \text { as } t \rightarrow 0^{+} .
\end{array}\right.
$$

Theorem 2.21 (67]). Let $\nu>8$. There exists a solution $\left(u, \partial_{t} u\right)$ of (FNLW) defined on the time interval $\left(0, T_{0}\right)$ such that

$$
\lim _{t \rightarrow 0^{+}}\left\|\left(u(t)-W_{\lambda(t)}-u_{0}^{*}, \partial_{t} u(t)-u_{1}^{*}\right)\right\|_{\dot{H}^{1} \times L^{2}}=0,
$$

where $\lambda(t)=t^{\nu+1}$, and $\left(u_{0}^{*}, u_{1}^{*}\right)$ is an explicit radial $C^{2}$ function.

We refer to the situation of Theorem 2.20 as the non-degenerate case and to the situation of Theorem 2.21 as the degenerate case. Note that in Theorem 2.20 we allow any regular $\left(u_{0}^{*}, u_{1}^{*}\right)$ with $u_{0}^{*}(0)>0$. The above result might be seen as a first step in a possible classification of all blow-up solutions with a non-degenerate asymptotic profile.

Let us mention that radiality is only a simplifying assumption. A similar construction should be possible also for non-radial $\left(u_{0}^{*}, u_{1}^{*}\right)$.

2.7. Description of type II blow up solutions. Duyckaerts-Kenig-Merle (JEMS, [37])

first studied the type II blow up solutions when the kinetic energy of solution for (FNLW) is slightly larger than the kinetic energy of ground state.

- If $d=3,4$ or $u$ is radial, and

$$
\sup _{t \in\left[0, T_{+}\right)}\left[\|\nabla u\|_{2}^{2}+\left\|\partial_{t} u\right\|_{2}^{2}\right]<\|\nabla W\|_{2}^{2} .
$$

Then $T_{+}=\infty$ and the solution scattering forward in time.

The threshold $\|\nabla W\|_{2}^{2}$ is sharp. Krieger, Schlag and Tataru [88]'s result shows: For $\forall \eta_{0}>0$, there exists a Type II blow-up solution such that

$$
\sup _{t \in\left[0, T_{+}\right)}\left[\|\nabla u\|_{2}^{2}+\left\|\partial_{t} u\right\|_{2}^{2}\right]<\|\nabla W\|_{2}^{2}+\eta_{0} .
$$

Duyckaerts-Kenig-Merle investigate the inverse problem under an appropriate small assumption.

Description theorem of type II non radial blow up solutions: 
Theorem 2.22 (Duyckaerts-Kenig-Merle, 38]). Let $d=3$ or $d=5$ and $\eta_{0}$ be small, the maximal solution $u:\left[0, T_{+}\right) \times \mathbb{R}^{3} \rightarrow \mathbb{R}$ for (FNLW) satisfies that $u:\left[0, T_{+}\right) \times \mathbb{R}^{3} \rightarrow \mathbb{R} T_{+}<+\infty$ and

$$
\sup _{t \in\left[0, T_{+}\right)}\left[\|\nabla u(t, \cdot)\|_{2}^{2}+\frac{d-2}{2}\left\|\partial_{t} u(t, \cdot)\right\|_{2}^{2}\right] \leq\|\nabla W\|_{2}^{2}+\eta_{0} .
$$

Then there exist $\left(v_{0}, v_{1}\right) \in \dot{H}^{1} \times L^{2}, \iota_{0} \in\{ \pm 1\}$, small parameter $\ell>0$ and $x(t), \lambda(t)$ with

$$
\lim _{t \rightarrow T_{+}} \frac{\lambda(t)}{T_{+}-t}=0, \lim _{t \rightarrow T_{+}} \frac{x(t)}{T_{+}-t}=\ell \vec{e}_{1},|\ell| \leq C \eta_{0}^{\frac{1}{4}}
$$

such that when $t \rightarrow T_{+}$,

$$
\left(u(t, \cdot), \partial_{t}(t, \cdot)\right)-\left(v_{0}, v_{1}\right)-\left(\frac{\iota_{0}}{\lambda(t)^{\frac{d-2}{2}}} W_{\ell}\left(0, \frac{x-x(t)}{\lambda(t)}\right), \frac{\iota_{0}}{\lambda(t)^{\frac{d}{2}}}\left(\partial_{t} W_{\ell}\right)\left(0, \frac{x-x(t)}{\lambda(t)}\right)\right) \rightarrow 0
$$

in $\dot{H}^{1} \times L^{2}$, where

$$
\left\{\begin{array}{l}
W_{\ell}(t, x)=\left(1+\frac{\left|x_{1}-\ell t\right|^{2}}{d(d-2)\left(1-\ell^{2}\right)}+\frac{|\bar{x}|^{2}}{d(d-2)}\right)^{-\frac{d-2}{2}} \\
W_{\ell}(t, x) \triangleq W\left(\frac{x_{1}-\ell t}{\sqrt{1-\ell^{2}}}, \bar{x}\right), \quad \text { Lorentz transform, } \\
\left\|\nabla v_{0}\right\|_{2}^{2}+\frac{d-2}{2}\left\|v_{1}\right\|_{2}^{2} \leq \eta_{0}, \quad\left(v_{0}, v_{1}\right) \text { to be small initial data. }
\end{array}\right.
$$

Remark 2.23. (i) The clue of proof: By introducing the regular part of $u(t, x)$ :

$$
v(t, x)=S_{N}\left(t-T_{+}\right)\left(v_{0}, v_{1}\right) \in C\left(\mathbb{R} ; \dot{H}^{1} \times L^{2}\right),
$$

where $\left(v_{0}, v_{1}\right)$ is a weak convergent point of $\left(u, \partial_{t} u\right)$. Then 2.42$)$ can be reduced to prove the singularity part $a(t, x)=u(t, x)-v(t, x)$ such that when $t \rightarrow T_{+}$, in the sense of $\dot{H}^{1} \times L^{2}$,

$$
\begin{aligned}
& \left(\lambda(t)^{\frac{d-2}{2}} a(t, \lambda(t) x+x(t)), \lambda(t)^{\frac{d}{2}} \partial_{t} a(t, \lambda(t) x+x(t))\right) \rightarrow \ell_{0}\left(W_{\ell}(0), \partial_{t} W_{\ell}(0)\right) . \\
& \Uparrow, \forall t_{n}^{\prime} \rightarrow T_{+} \text {, } \\
& \left(\lambda\left(t_{n}^{\prime}\right)^{\frac{d-2}{2}} a\left(t_{n}^{\prime}, \lambda\left(t_{n}^{\prime}\right) x+x\left(t_{n}^{\prime}\right)\right), \lambda\left(t_{n}^{\prime}\right)^{\frac{d}{2}} \partial_{t} a\left(t_{n}^{\prime}, \lambda\left(t_{n}^{\prime}\right) x+x\left(t_{n}^{\prime}\right)\right)\right) \rightarrow \ell_{0}\left(W_{\ell}(0), \partial_{t} W_{\ell}(0)\right), \\
& \Uparrow \exists t_{n} \rightarrow T_{+} \text {, } \\
& \left(\lambda\left(t_{n}\right)^{\frac{d-2}{2}} a\left(t_{n}, \lambda\left(t_{n}\right) x+x\left(t_{n}\right)\right), \lambda\left(t_{n}\right)^{\frac{d}{2}} \partial_{t} a\left(t_{n}, \lambda\left(t_{n}\right) x+x\left(t_{n}\right)\right)\right) \rightarrow \ell_{0}\left(W_{\ell}(0), \partial_{t} W_{\ell}(0)\right), \\
& \left(\lambda\left(t_{n}\right)^{\frac{d-2}{2}} a\left(t_{n}, \lambda\left(t_{n}\right) x+x\left(t_{n}\right)\right), \lambda\left(t_{n}\right)^{\frac{d}{2}} \partial_{t} a\left(t_{n}, \lambda\left(t_{n}\right) x+x\left(t_{n}\right)\right)\right) \rightarrow \ell_{0}\left(W_{\ell}(0), \partial_{t} W_{\ell}(0)\right),
\end{aligned}
$$

for details please refer to 38 .

(ii) It seems to be interesting for us to study the description of type II blow up solutions of focusing energy critical wave equation in higher dimensions.

2.8. Bounds on the speed of type II blow-up. In sum, for type II blowup solutions,

Theorem 2.24 (37, 38, 23]). Let $u(t)$ be a radial solution of (FNLW) which blows up at $t=T_{+}$by concentration of one bubble of energy. Then there exist $\vec{u}_{0}^{*} \in \dot{H}^{1} \times L^{2}$ and $\lambda(t) \in C\left(\left[t_{0}, T_{+}\right),(0,+\infty)\right)$ such that

$$
\lim _{t \rightarrow T_{+}}\left\|\vec{u}(t)-\vec{W}_{\lambda(t)}-\vec{u}_{0}^{*}\right\|_{\dot{H}^{1} \times L^{2}}=0, \quad \lim _{t \rightarrow T_{+}}\left(T_{+}-t\right)^{-1} \lambda(t)=0 .
$$


In the following, the function $\vec{u}_{0}^{*}$ is called the asymptotic profile. Note that in 38 . a more general, non-radial version of the above theorem was proved for $d \in\{3,5\}$.

Solutions verifying (2.44) were first constructed in dimension $d=3$ by Krieger, Schlag and Tataru 88, who obtained all possible polynomial blow-up rates $\lambda(t) \sim$ $\left(T_{+}-t\right)^{1+\nu}, \nu>0$. For $d=4$ smooth solutions blowing up at a particular rate were constructed by Hillairet and Raphaël [62. For $d=5$ they proved in 67] that for any radially symmetric asymptotic profile $\vec{u}_{0}^{*} \in H^{4} \times H^{3}$ such that $u_{0}^{*}(0)>0$, there exists a solution $\vec{u}(t)$ such that $(2.44)$ holds. For these solutions the concentration speed of the bubble is

$$
\lambda(t) \sim u_{0}^{*}(0)^{2}\left(T_{+}-t\right)^{4} .
$$

Jendrej continued the investigation of the relationship between the behaviour of $\vec{u}_{0}^{*}$ at $x=0$ and possible blow-up speeds, still in the special case when the asymptotic profile $\vec{u}_{0}^{*}$ is sufficiently regular. He proved the following result.

Theorem 2.25 (Bounds on the speed of type II blow-up, 68]). Let $d \in\{3,4,5\}$ and $s>\frac{d-2}{2}, s \geq 1$. Let $\vec{u}_{0}^{*}=\left(u_{0}^{*}, \dot{u}_{0}^{*}\right) \in H^{s+1} \times H^{s}$ be a radial function. Suppose that $\vec{u}$ is a radial solution of (FNLW) such that

$$
\lim _{t \rightarrow T_{+}}\left\|\vec{u}(t)-\vec{W}_{\lambda(t)}-\vec{u}_{0}^{*}\right\|_{\dot{H}^{1} \times L^{2}}=0, \quad \lim _{t \rightarrow T_{+}} \lambda(t)=0, \quad T_{+}<+\infty .
$$

There exists a constant $C>0$ depending on $\vec{u}_{0}^{*}$ such that:

- If $d \in\{4,5\}$, then for $T_{+}-t$ sufficiently small there holds

$$
\lambda(t) \leq C\left(T_{+}-t\right)^{\frac{4}{6-d}} .
$$

- If $d=3$, then there exists a sequence $t_{n} \rightarrow T_{+}$such that

$$
\lambda\left(t_{n}\right) \leq C\left(T_{+}-t_{n}\right)^{\frac{4}{6-d}} .
$$

Theorem 2.26 ([68]). Let $d \in\{3,4,5\}$. Let $\vec{u}_{0}^{*}=\left(u_{0}^{*}, \dot{u}_{0}^{*}\right) \in H^{3} \times H^{2}$ be a radial function such that

$$
u_{0}^{*}(0)<0 \text {. }
$$

There exist no radial solutions of (FNLW) such that

$$
\lim _{t \rightarrow T_{+}}\left\|\vec{u}(t)-\vec{W}_{\lambda(t)}-\vec{u}_{0}^{*}\right\|_{\dot{H}^{1} \times L^{2}}=0, \quad \lim _{t \rightarrow T_{+}} \lambda(t)=0, \quad T_{+}<+\infty .
$$

2.9. Soliton resolution conjecture. From the above analysis, we can obtain the dynamics of the energy solutions to the focusing energy-critical wave equation in some restrictions. Soliton resolution conjecture describes such dynamics. In [130, Soffer first proposed this conjecture, and lately it was modified as following

Conjecture 2.27 (Soliton resolution conjecture). In the context of equation (NLW), the soliton resolution conjecture predicts that any bounded solution should asymptotically decouple into a finite sum of modulated solitons, a regular part in the finite time blow up case or a free radiation in the global case, plus a residue term that vanishes asymptotically in the energy space as time approaches the maximal time of existence.

Now, we give the precise soliton resolution for focusing energy-critical NLW. Let Lorentz transforms of $W$

$$
W_{\ell}(t, x) \triangleq W\left(\left(-\frac{t}{\sqrt{1-|\ell|^{2}}}+\frac{1}{|\ell|^{2}}\left(\frac{1}{\sqrt{1-|\ell|^{2}}}-1\right) \ell \cdot x\right) \ell+x\right) .
$$


Note that

$$
W_{\ell}(t, x)=W_{\ell}(0, x-t \ell), \quad \ell \in \mathbb{R}^{d},|\ell|<1,
$$

so that $W_{\ell}$ is a solitary wave traveling at speed $|\ell|$. The energy of $W_{\ell}$ is given by:

$$
E\left(\vec{W}_{\ell}(0)\right)=\frac{1}{\sqrt{1-|\ell|^{2}}} E(\vec{W}(0)) \underset{|\ell| \rightarrow 1}{\longrightarrow}+\infty .
$$

It is conjectured that any bounded solution of focusing energy-critical NLW is a sum of modulated, decoupled traveling waves and a scattering part. More precisely:

Conjecture 2.28 (Soliton resolution for general type II blowup solutions). Let $u$ be a solution of of $(\mathrm{FNLW})$ on $\left[0, T_{+}\right) \times \mathbb{R}^{d}$ such that

$$
\sup _{t \in\left[0, T_{+}\right)}\|\vec{u}(t)\|_{\dot{H}^{1} \times L^{2}}<\infty .
$$

(i) If $T_{+}=+\infty$, then there exist a solution $v_{L}$ of the linear wave equation

$$
\left\{\begin{array}{l}
\left(\partial_{t}^{2}-\Delta\right) v_{L}=0, \\
\left.\vec{v}_{L}\right|_{t=0}=\left(v_{0}, v_{1}\right) \in \dot{H}^{1} \times L^{2},
\end{array}\right.
$$

an integer $J \geq 0$, and for $j \in\{1, \ldots, J\}$, a (nonzero) traveling wave $W_{\ell_{j}}^{j}\left(\left|\ell_{j}\right|<1\right.$ ), and parameters $x_{j}(t) \in \mathbb{R}^{d}, \lambda_{j}(t) \in \mathbb{R}^{+}$such that

$\lim _{t \rightarrow+\infty} \vec{u}(t)-\vec{v}_{L}(t)-\sum_{j=1}^{J}\left(\frac{1}{\lambda_{j}(t)^{\frac{N-2}{2}}} W_{\ell_{j}}^{j}\left(0, \frac{\cdot-x_{j}(t)}{\lambda_{j}(t)}\right), \frac{1}{\lambda_{j}(t)^{\frac{N}{2}}} \partial_{t} W_{\ell_{j}}^{j}\left(0, \frac{\cdot-x_{j}(t)}{\lambda_{j}(t)}\right)\right)=0$

in $\dot{H}^{1} \times L^{2}$ and

$$
\left\{\begin{array}{l}
\forall j \in\{1, \ldots, J\}, \quad \lim _{t \rightarrow \infty} \frac{x_{j}(t)}{t}=\ell_{j}, \quad \lim _{t \rightarrow \infty} \frac{\lambda_{j}(t)}{t}=0 \\
\forall j, k \in\{1, \ldots, J\}, \quad j \neq k \Longrightarrow \lim _{t \rightarrow+\infty} \frac{\left|x_{j}(t)-x_{k}(t)\right|}{\lambda_{j}(t)}+\frac{\lambda_{j}(t)}{\lambda_{k}(t)}+\frac{\lambda_{k}(t)}{\lambda_{j}(t)}=+\infty \\
W_{\ell}(t, x)=W\left(\left(-\frac{t}{\sqrt{1-|\ell|^{2}}}+\frac{1}{|\ell|^{2}}\left(\frac{1}{\sqrt{1-|\ell|^{2}}}-1\right) \ell \cdot x\right) \ell+x\right) .
\end{array}\right.
$$

(ii) If $T_{+}<+\infty$, then the following is true. Define the singular set

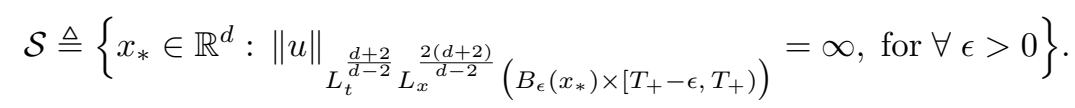

Then $\mathcal{S}$ is a finite set. Let $x_{*} \in \mathcal{S}$ be a singular point. Then there exist an integer $J_{*} \geq 1, r_{*}>0, \vec{v}_{0} \in \dot{H}^{1} \times L^{2}$, scales $\lambda_{n}^{j}$ with $0<\lambda_{n}^{j} \ll T_{+}-t_{n}$, positions $c_{n}^{j} \in R^{d}$ satisfying $c_{n}^{j} \in B_{\beta\left(T_{+}-t\right)}\left(x_{*}\right)$ for some $\beta \in(0,1)$ with $\ell_{j}=\lim _{n \rightarrow \infty} \frac{c_{n}^{j}-x_{*}}{T_{+}-t}$ well defined, and traveling waves $W_{\ell_{j}}^{j}$, for $1 \leq j \leq J_{*}$, such that inside the ball $B_{r_{*}}\left(x_{*}\right)$ we have

$$
\begin{aligned}
\vec{u}\left(t_{n}\right)=\vec{v}_{0}+ & \sum_{j=1}^{J_{*}}\left(\left(\lambda_{n}^{j}\right)^{-\frac{d}{2}+1} W_{\ell_{j}}^{j}\left(\frac{x-c_{n}^{j}}{\lambda_{n}^{j}}, 0\right),\left(\lambda_{n}^{j}\right)^{-\frac{d}{2}} \partial_{t} W_{\ell_{j}}^{j}\left(\frac{x-c_{n}^{j}}{\lambda_{n}^{j}}, 0\right)\right) \\
& +o_{\dot{H}^{1} \times L^{2}}(1), \quad \text { as } n \rightarrow \infty .
\end{aligned}
$$

In addition, the parameters $\lambda_{n}^{j}, c_{n}^{j}$ satisfy the pseudo-orthogonality condition

$$
\frac{\lambda_{n}^{j}}{\lambda_{n}^{j^{\prime}}}+\frac{\lambda_{n}^{j^{\prime}}}{\lambda_{n}^{j}}+\frac{\left|c_{n}^{j}-c_{n}^{j^{\prime}}\right|}{\lambda_{n}^{j}} \rightarrow \infty
$$


as $n \rightarrow \infty$, for each $1 \leq j \neq j^{\prime} \leq J_{*}$.

Remark 2.29. The soliton resolution conjecture was proved in [39], in the radial case for $d=3$ as in Theorem 2.30 below. For other dimensions in the radial case, soliton resolution is only known along a sequence of times, see [23, 117, 69]. In the nonradial case, the soliton resolution was proved for type II blow up solutions in [38] for $d=3,5$, under an extra smallness condition. For general large data (does not scatter forward in time), it was proved in 42 that along a sequence of times, the solution converges locally, after an appropriate rescaling, to a modulated soliton. The proof of 42 relies on a compactness/rigidity argument that works for a large class of dispersive equations.

Recently, Duyckaerts-Jia-Kenig-Merle [36] (see Theorem 2.31 bleow) prove the soliton resolution conjecture for general type II solutions along a sequence of times. This is an important step towards the full soliton resolution in the nonradial case and without any size restrictions.

If $f$ and $g$ are two positive functions defined in a neighborhood of $\ell \in \mathbb{R} \cup\{ \pm \infty\}$, we will write

$$
f(t) \ll g(t) \text { as } t \rightarrow \ell \text { if and only if } \lim _{t \rightarrow \ell} \frac{f(t)}{g(t)}=0 .
$$

Soliton resolution conjecture for radial solution in $\mathbb{R}^{3}$ was proved by DuyckaertsKenig-Merle [39].

Theorem 2.30 (Soliton resolution conjecture for radial solution in $\mathbb{R}^{3},[39]$ ). Let $u$ be a radial solution of (FNLW) and $T_{+}=T_{+}(u)$. Then one of the following holds:

- Type I blow-up: $T_{+}<\infty$ and

$$
\lim _{t \rightarrow T_{+}} \|\left(u(t), \partial_{t} u(t) \|_{\dot{H}^{1} \times L^{2}}=+\infty .\right.
$$

- Type II blow-up: $T_{+}<\infty$ and there exist $\left(v_{0}, v_{1}\right) \in \dot{H}^{1} \times L^{2}$, an integer $J \in \mathbb{N} \backslash\{0\}$, and for all $j \in\{1, \ldots, J\}$, a sign $\iota_{j} \in\{ \pm 1\}$, and a positive function $\lambda_{j}(t)$ defined for $t$ close to $T_{+}$such that

$$
\begin{gathered}
\lambda_{1}(t) \ll \lambda_{2}(t) \ll \ldots \ll \lambda_{J}(t) \ll T_{+}-t \text { as } t \rightarrow T_{+} \\
\lim _{t \rightarrow T_{+}}\left\|\left(u(t), \partial_{t} u(t)\right)-\left(v_{0}+\sum_{j=1}^{J} \frac{\iota_{j}}{\lambda_{j}^{1 / 2}(t)} W\left(\frac{x}{\lambda_{j}(t)}\right), v_{1}\right)\right\|_{\dot{H}^{1} \times L^{2}}=0 .
\end{gathered}
$$

- Global solution: $T_{+}=+\infty$ and there exist a solution $v_{L}$ of the linear wave equation, an integer $\mathbb{N} \backslash\{0\}$, and for all $j \in\{1, \ldots, J\}$, a sign $\iota_{j} \in$ $\{ \pm 1\}$, and a positive function $\lambda_{j}(t)$ defined for large $t$ such that

$$
\begin{gathered}
\lambda_{1}(t) \ll \lambda_{2}(t) \ll \ldots \ll \lambda_{J}(t) \ll t \text { as } t \rightarrow+\infty \\
\lim _{t \rightarrow+\infty}\left\|\left(u(t), \partial_{t} u(t)\right)-\left(v_{L}(t)+\sum_{j=1}^{J} \frac{\iota_{j}}{\lambda_{j}^{1 / 2}(t)} W\left(\frac{x}{\lambda_{j}(t)}\right), \partial_{t} v_{L}(t)\right)\right\|_{\dot{H}^{1} \times L^{2}}=0 .
\end{gathered}
$$

Soliton resolution conjecture along a sequence of times was solved by DuyckaertsJia-Kenig-Merle 36 . 
Theorem 2.31 (Soliton resolution conjecture along a sequence of times, [36]). Let $\left(u, \partial_{t} u\right) \in C\left(\left[0, T_{+}\right), \dot{H}^{1} \times L^{2}\left(\mathbb{R}^{d}\right)\right)$ with $u \in L_{t}^{\frac{d+2}{d-2}} L_{x}^{\frac{2(d+2)}{d-2}}\left(\mathbb{R}^{d} \times[0, T)\right)$ for any $T<$ $T_{+}$, be a solution to (FNLW) that satisfies

$$
\sup _{t \in\left[0, T_{+}\right)}\left\|\left(u, \partial_{t} u\right)(t)\right\|_{\dot{H}^{1} \times L^{2}}<\infty
$$

where $T_{+}$denotes the maximal existence time of $u$.

Case I: $T_{+}<\infty$. Define the singular set

$$
\mathcal{S} \triangleq\left\{x_{*} \in \mathbb{R}^{d}:\|u\|_{L_{t}^{\frac{d+2}{d-2}} L_{x}^{\frac{2(d+2)}{d-2}}\left(B_{\epsilon}\left(x_{*}\right) \times\left[T_{+}-\epsilon, T_{+}\right)\right)}=\infty, \text { for, } \forall \epsilon>0\right\} .
$$

Then $\mathcal{S}$ is a finite set. Let $x_{*} \in \mathcal{S}$ be a singular point. Then there exist an integer $J_{*} \geq 1, r_{*}>0, \vec{v}_{0} \in \dot{H}^{1} \times L^{2}$, a time sequence $t_{n} \uparrow T_{+}$, scales $\lambda_{n}^{j}$ with $0<\lambda_{n}^{j} \ll$ $T_{+}-t_{n}$, positions $c_{n}^{j} \in \mathbb{R}^{d}$ satisfying $c_{n}^{j} \in B_{\beta\left(T_{+}-t_{n}\right)}\left(x_{*}\right)$ for some $\beta \in(0,1)$ with $\ell_{j}=\lim _{n \rightarrow \infty} \frac{c_{n}^{j}-x_{*}}{T_{+}-t_{n}}$ well defined, and traveling waves $W_{\ell_{j}}^{j}$, for $1 \leq j \leq J_{*}$, such that inside the ball $B_{r_{*}}\left(x_{*}\right)$ we have

$$
\begin{aligned}
\vec{u}\left(t_{n}\right)=\vec{v}_{0}+\sum_{j=1}^{J_{*}} & \left(\left(\lambda_{n}^{j}\right)^{-\frac{d}{2}+1} W_{\ell_{j}}^{j}\left(\frac{x-c_{n}^{j}}{\lambda_{n}^{j}}, 0\right),\left(\lambda_{n}^{j}\right)^{-\frac{d}{2}} \partial_{t} W_{\ell_{j}}^{j}\left(\frac{x-c_{n}^{j}}{\lambda_{n}^{j}}, 0\right)\right) \\
& +o_{\dot{H}^{1} \times L^{2}}(1), \quad \text { as } n \rightarrow \infty .
\end{aligned}
$$

In addition, the parameters $\lambda_{n}^{j}, c_{n}^{j}$ satisfy the pseudo-orthogonality condition

$$
\frac{\lambda_{n}^{j}}{\lambda_{n}^{j^{\prime}}}+\frac{\lambda_{n}^{j^{\prime}}}{\lambda_{n}^{j}}+\frac{\left|c_{n}^{j}-c_{n}^{j^{\prime}}\right|}{\lambda_{n}^{j}} \rightarrow \infty,
$$

as $n \rightarrow \infty$, for each $1 \leq j \neq j^{\prime} \leq J_{*}$.

Case II: $T_{+}=\infty$. There exist a finite energy solution $u^{L}$ to the linear wave equation

$$
\partial_{t t} u^{L}-\Delta u^{L}=0 \text { in } \mathbb{R}^{d} \times \mathbb{R},
$$

an integer $J_{*} \geq 02$, a time sequence $t_{n} \uparrow \infty$, scales $\lambda_{n}^{j}$ with $\lambda_{n}^{j}>0$ and $\lim _{n \rightarrow \infty} \frac{\lambda_{n}^{j}}{t_{n}}=0$, positions $c_{n}^{j} \in \mathbb{R}^{d}$ satisfying $c_{n}^{j} \in B_{\beta t_{n}}(0)$ for some $\beta \in(0,1)$ with $\ell_{j}=\lim _{n \rightarrow \infty} \frac{c_{n}^{j}}{t_{n}}$ well defined, and traveling waves $W_{\ell_{j}}^{j}$, for $1 \leq j \leq J_{*}$, such that

$$
\begin{aligned}
\vec{u}\left(t_{n}\right)=\vec{u}^{L}\left(t_{n}\right)+\sum_{j=1}^{J_{*}} & \left(\left(\lambda_{n}^{j}\right)^{-\frac{d}{2}+1} W_{\ell_{j}}^{j}\left(\frac{x-c_{n}^{j}}{\lambda_{n}^{j}}, 0\right),\left(\lambda_{n}^{j}\right)^{-\frac{d}{2}} \partial_{t} W_{\ell_{j}}^{j}\left(\frac{x-c_{n}^{j}}{\lambda_{n}^{j}}, 0\right)\right) \\
& +o_{\dot{H}^{1} \times L^{2}}(1), \quad \text { as } n \rightarrow \infty .
\end{aligned}
$$

In addition, the parameters $\lambda_{n}^{j}, c_{n}^{j}$ satisfy the pseudo-orthogonality condition 2.67).

\footnotetext{
${ }^{2}$ If $J_{*}=0$, then there is no soliton in the decomposition below and the solution scatters.
} 


\subsection{Blowup at infinity for the critical wave equation.}

Theorem 2.32 (31]). There exists an $\varepsilon_{0}>0$ such that for any $\delta>0$ and $\mu \in \mathbb{R}$ with $|\mu| \leq \varepsilon_{0}$ there exists a $t_{0} \geq 1$ and an energy class solution $u:\left[t_{0}, \infty\right) \times \mathbb{R}^{3} \rightarrow \mathbb{R}$ of (FNLW) of the form

$$
u(t, x)=t^{\frac{\mu}{2}} W\left(t^{\mu} x\right)+\eta(t, x), \quad|x| \leq t, t \geq t_{0}
$$

and for all $t \geq t_{0}$

$$
\left\{\begin{array}{l}
\left\|\partial_{t} u(t, \cdot)\right\|_{L^{2}\left(\mathbb{R}^{3} \backslash B_{t}\right)}+\|\nabla u(t, \cdot)\|_{L^{2}\left(\mathbb{R}^{3} \backslash B_{t}\right)} \leq \delta, \\
\left\|\partial_{t} \eta(t, \cdot)\right\|_{L^{2}\left(B_{t}\right)}+\|\nabla \eta(t, \cdot)\|_{L^{2}\left(B_{t}\right)} \leq \delta
\end{array}\right.
$$

where $B_{t}:=\left\{x \in \mathbb{R}^{3}:|x|<t\right\}$.

It seemed plausible to expect a strong version of the soliton resolution conjecture to hold.

\subsection{Two-bubble solutions.}

Theorem 2.33 (Two-bubble solutions, [65]). Let $d=6$. There exists a solution $\vec{u}:\left(-\infty, T_{0}\right] \rightarrow \dot{H}^{1} \times L^{2}$ of (FNLW) such that

$$
\lim _{t \rightarrow-\infty}\left\|\vec{u}(t)-\left(\vec{W}+\vec{W}_{\frac{1}{\kappa} e^{-\kappa|t|} \mid}\right)\right\|_{\dot{H}^{1} \times L^{2}}=0, \quad \text { with } \kappa \triangleq \sqrt{5 / 4},
$$

where $\vec{W}=(W, 0)$ and

$$
\vec{W}_{\lambda}=\left(W_{\lambda}, 0\right), W_{\lambda}(x)=\frac{1}{\lambda^{2}} W(x / \lambda) .
$$

Remark 2.34. (i) More precisely, Jendrej in 65] proved that

$$
\left\|\vec{u}(t)-\left(\vec{W}+\vec{W}_{\frac{1}{\kappa} e^{-\kappa|t|}}\right)\right\|_{\dot{H}^{1} \times L^{2}} \leq C_{1} \cdot e^{-\frac{1}{2} \kappa|t|}
$$

for some constant $C_{1}>0$.

(ii) The author construct here pure two-bubbles, that is the solution approaches a superposition of two stationary states, with no energy transformed into radiation. By the conservation of energy and the decoupling of the two bubbles, we necessarily have $E(\vec{u}(t))=2 E(\vec{W})$. Pure one-bubble cannot concentrate and is completely classified, see 43 .

(iii) It was proved in 66] (see Theorem 2.35 below), in any dimension $d \geq 3$, that there exist no solutions $\vec{u}(t):\left[t_{0}, T_{+}\right) \rightarrow \dot{H}^{1} \times L^{2}$ of (FNLW) such that $\left\|\vec{u}(t)-\left(\vec{W}_{\mu(t)}-\vec{W}_{\lambda(t)}\right)\right\|_{\dot{H}^{1} \times L^{2}} \rightarrow 0$ with $\lambda(t) \ll \mu(t)$ as $t \rightarrow T_{+} \leq+\infty$.

(iv) In any dimension $d>6$ one can expect an analogous result with concentration rate $\lambda(t) \sim|t|^{-\frac{4}{d-6}}$.

According to Theorem 2.30, for $d=3$ such a solution has to behave asymptotically as a decoupled superposition of stationary states. Such solutions are called (pure) multi-bubbles (or $n$-bubbles, where $n$ is the number of bubbles). By conservation of energy, if $\vec{u}(t)$ is an $n$-bubbles, then

$$
E(\vec{u}(t))=n E(\vec{W}) .
$$

The case $n=1$ in dimension $d \in\{3,4,5\}$ was treated by Duyckaerts and Merle [43, who obtained a complete classification of solutions of (FNLW) at energy level $E(\vec{u}(t))=E(\vec{W})$. 
Theorem 2.35 (Nonexistence of radial two-bubbles with opposite signs, 66]). Let $d \geq 3$. There exists no radial solutions $\vec{u}:\left[t_{0}, T_{+}\right) \rightarrow \dot{H}^{1} \times L^{2}$ of (FNLW) such that

$$
\lim _{t \rightarrow T_{+}}\left\|\vec{u}(t)-\vec{W}_{\lambda_{1}(t)}+\vec{W}_{\lambda_{2}(t)}\right\|_{\dot{H}^{1} \times L^{2}}=0
$$

and

- The case $T_{+}<+\infty: \lambda_{1}(t) \ll \lambda_{2}(t) \ll T_{+}-t$ as $t \rightarrow T_{+}$,

- The case $T_{+}=+\infty: \lambda_{1}(t) \ll \lambda_{2}(t) \ll t$ as $t \rightarrow+\infty$.

2.12. Global dynamics away from the ground state. Denote

$$
\vec{u}:=(|\nabla| u, \dot{u}) \in L_{\mathrm{rad}}^{2}\left(\mathbb{R}^{d}\right)^{2} \triangleq \mathcal{H}
$$

and

and

$$
\mathcal{S} \triangleq\left\{W_{\lambda}\right\}_{\lambda>0}, \quad W_{\lambda}=T_{\lambda} W(x)=\lambda^{d / 2-1} W(\lambda x),
$$

$$
J(\varphi):=\int_{\mathbb{R}^{d}}\left[\left.\frac{1}{2}\left|\nabla \varphi^{2}-\frac{1}{2^{*}}\right| \varphi\right|^{2^{*}}\right] d x .
$$

Theorem 2.36 (Global dynamics away from the ground state, [85]). There exist a small $\epsilon_{*}>0$, a neighborhood $\mathcal{B}$ of $\overrightarrow{\mathcal{S}}$ within $O\left(\epsilon_{*}\right)$ distance in $\mathcal{H}$, and a continuous functional

$$
\mathcal{G}:\left\{\vec{\varphi} \in \mathcal{H} \backslash \mathcal{B} \mid E(\vec{\varphi})<J(W)+\epsilon_{*}^{2}\right\} \rightarrow\{ \pm 1\},
$$

such that the following properties hold: For any solution $u$ with $E(\vec{u})<J(W)+\epsilon_{*}^{2}$ on the maximal existence interval $I(u)$, let

$$
\begin{aligned}
I_{0}(u) & :=\{t \in I(u) \mid \vec{u}(t) \in \mathcal{B}\}, \\
I_{ \pm}(u) & :=\{t \in I(u) \mid \vec{u}(t) \notin \mathcal{B}, \mathcal{G}(\vec{u}(t))= \pm 1\} .
\end{aligned}
$$

Then $I_{0}(u)$ is an interval, $I_{+}(u)$ consists of at most two infinite intervals, and $I_{-}(u)$ consists of at most two finite intervals. $u(t)$ scatters to 0 as $t \rightarrow \pm \infty$ if and only if $\pm t \in I_{+}(u)$ for large $t>0$. Moreover, there is a uniform bound $M<\infty$ such that

$$
\|u\|_{L_{t, x}^{q}\left(I_{+}(u) \times \mathbb{R}^{d}\right)} \leq M, q:=\frac{2(d+1)}{d-2} .
$$

For each $\sigma_{1}, \sigma_{2} \in\{ \pm\}$, let $A_{\sigma_{1}, \sigma_{2}}$ be the collection of initial data $\vec{u}(0) \in \mathcal{H}$ such that $E(\vec{u})<J(W)+\epsilon_{*}^{2}$, and for some $T_{-}<0<T_{+}$,

$$
\left(-\infty, T_{-}\right) \cap I(u) \subset I_{\sigma_{1}}(u),\left(T_{+}, \infty\right) \cap I(u) \subset I_{\sigma_{2}}(u) .
$$

Then each of the four sets $A_{ \pm, \pm}$is open and non-empty, exhibiting all possible combinations of scattering to zero/finite time blowup as $t \rightarrow \pm \infty$, respectively.

2.13. Unconditional uniqueness. Consider energy-critical NLW

$$
\left\{\begin{array}{l}
u_{t t}-\Delta u=\mu|u|^{\frac{4}{d-2}} u, \\
\left(u, \partial_{t} u\right)(0)=\left(u_{0}, u_{1}\right) \in \dot{H}^{1} \times L^{2} .
\end{array}\right.
$$

It is well known that this equation is locally well-posedness in the energy space, $u \in C_{t}\left(\dot{H}^{1}\right)$. Furthermore, this solution can be extended to a global one in the defocusing case (i.e. $\mu=-1$ ). However uniqueness holds only with some additional assumptions, like $u \in L_{t, x}^{\frac{2(d+1)}{d-2}}$ (strong Strichartz solution). This restriction can be related to the choice of spaces involved in the fixed point argument. Therefore, it is natural to ask whether such assumptions are necessary to provide uniqueness, 
or if uniqueness in the energy class holds. Uniqueness in the energy class for the defocusing case is known to hold under an additional a priori assumption of type $\partial_{t} u \in L_{t}^{1} L_{x}^{4}$ (or assuming smoothness on $u$, see [136]). By unconditional uniqueness, we mean that for given initial data $\left(u_{0}, u_{1}\right)$, there exists at most one solution of 2.72 in the class $C_{t} \dot{H}_{x}^{1}\left(I \times \mathbb{R}^{d}\right)$,

Unconditional uniqueness
\begin{tabular}{|c|c|c|}
\hline$d=3$ & $d=4,5$ & $d \geq 6$ \\
\hline Open! & Planchon [115] & Bulut-et al [14] \\
\hline
\end{tabular}

We note that we do not need any assumption on $\partial_{t} u$.

Remark 2.37. (i) We should also stress that the unconditional uniqueness in $d=3$ is still open due to the failure of the endpoint Strichartz estimates except for the radial case. However, Masmoudi-Planchon in 96 proved an interesting result concerning uniqueness of weak solutions $\left(u \in L_{t}^{\infty} \dot{H}^{1} \cap \dot{W}_{t}^{1,+\infty}\left(L_{x}^{2}\right)\right)$ to defocusing NLW in $\mathbb{R}^{3}$ under a local energy inequality assumption on the light cone as follows: for all $0 \leq s \leq t \leq t_{0}$

$$
\int_{B\left(x_{0}, t_{0}-t\right)} e(u(t, x)) d x \leq C \int_{B\left(x_{0}, \alpha\left(t_{0}-s\right)\right)} e(u(s, x)) d x
$$

and

$$
\frac{1}{\sqrt{2}} \int_{t_{1}}^{t^{\prime}} \int_{\partial B\left(x_{1}, \tau-t_{1}\right)} \frac{\left|\partial_{K_{1}} u(\tau)\right|^{2}}{2}+\frac{|u(\tau)|^{6}}{6} d \sigma d \tau \leq C \int_{B\left(x_{1}, \alpha\left(t^{\prime}-t_{1}\right)\right)} e\left(u\left(t^{\prime}, x\right)\right) d x
$$

where $C \geq 1, \alpha \geq 1$ and

$$
e(u)=|\partial u|^{2} / 2+|u|^{6} / 6, \quad K_{1}=\left\{\left|x-x_{1}\right|=t-t_{1}\right\} .
$$

In many cases we can only construct global weak solutions by using compactness arguments, but their uniqueness is not known. The weak-strong uniqueness investigation is an attempt to reconcile the weak and strong viewpoints of solutions. More precisely, this investigation is to show that any weak solution agrees with the strong solution sharing the same initial data if it exists. See, for instance, [136, 137] for wave and Schrödinger equations, [17, 18, 52, 90, 99] for the Navier-Stokes system, [50] for nonhomogeneous Navier-Stokes system and [51] for the isentropic compressible Navier-Stokes system.

(ii) In the context of $\dot{H}^{s}$ critical NLS, the unconditional uniqueness was first established by Furioli and Terraneo [47] using para-product analysis. A review of the unconditional uniqueness for both the NLS and NLW can be found in the paper by Furioli, Planchon and Terraneo [48.

2.14. Ill-posedness. For the ill-posedness, we refer to Christ, Colliander, Tao [19].

\section{ENERGY-SUPCRITICAL NLW}

Let us recall the classical results for the nonlinear wave equation(NLW).

$$
(\mathrm{NLW}) \quad \begin{cases}\partial_{t t} u-\Delta u+\mu|u|^{p-1} u=0, & (t, x) \in \mathbb{R} \times \mathbb{R}^{d} \\ \left(u, \partial_{t} u\right)(0, x)=\left(u_{0}, u_{1}\right)(x), & x \in \mathbb{R}^{d}\end{cases}
$$


where $u: \mathbb{R}_{t} \times \mathbb{R}_{x}^{d} \rightarrow \mathbb{R}$. The equation 3.1 can be classified energy-subcritical, energy-critical and energy-supercritical such as

$$
\begin{cases}p<1+\frac{4}{d-2}, & \text { energy-subcritical; } \\ p=1+\frac{4}{d-2}, & \text { energy-critical; } \\ p>1+\frac{4}{d-2}, & \text { energy-supcritical. }\end{cases}
$$

The energy-critical equations have been the most widely studied instances of NLW, since the rescaling

$$
\left(u, u_{t}\right)(t, x) \mapsto\left(\lambda^{\frac{2}{p-1}} u(\lambda t, \lambda x), \lambda^{\frac{2}{p-1}+1} u_{t}(\lambda t, \lambda x)\right), \quad p=1+\frac{4}{d-2}
$$

leaves invariant the energy of solutions, which is defined by

$$
E\left(u, u_{t}\right)=\frac{1}{2} \int_{\mathbb{R}^{d}}\left(\left|\partial_{t} u\right|^{2}+|\nabla u|^{2}\right) \mathrm{d} x+\frac{\mu}{p+1} \int_{\mathbb{R}^{d}}|u|^{p+1} \mathrm{~d} x,
$$

and is a conserved quantity for equation in (3.1). For the defocusing energy-critical NLW (3.1) with $\mu=1$, Grillakis [58 proved that the Cauchy problem of (3.1) with the initial data $\dot{H}^{1}\left(\mathbb{R}^{3}\right) \times L^{2}\left(\mathbb{R}^{3}\right)$ is globally well-posed, we refer the readers to 3 , [72], [124] and [139] for the scattering theory and the high dimensional case. In particular, Tao derived a exponential type spacetime bound in [139. In the above papers, their methods rely heavily on the classical finite speed of propagation (i.e. the monotonic local energy estimate on the light cone)

$$
\int_{|x| \leq R-t} e(t, x) d x \leq \int_{|x| \leq R} e(0, x) d x, t>0
$$

where

$$
e(t, x):=\frac{1}{2}\left|u_{t}\right|^{2}+\frac{1}{2}|\nabla u|^{2}+\frac{d-2}{2 d}|u|^{\frac{2 d}{d-2}},
$$

and Morawetz estimate

$$
\int_{\mathbb{R}} \int_{\mathbb{R}^{d}} \frac{|u|^{2^{*}}}{|x|} d x d t \leq C\left(E\left(u_{0}, u_{1}\right)\right), \quad 2^{*} \triangleq \frac{2 d}{d-2} .
$$

However, the Morawetz estimate fails for the focusing energy-critical NLW. Kenig and Merle [7] first employed sophisticated "concentrated compactness + rigidity method" to obtain the dichotomy-type result under the assumption that $E\left(u_{0}, u_{1}\right)<E(W, 0)$, where $W$ denotes the ground state of the elliptic equation

$$
\Delta W+|W|^{\frac{4}{d-2}} W=0
$$

Thereafter, Bulut et.al [14] extended the above result in [77] to higher dimensions. This was proven by making use of minimal counterexamples derived from the concentration-compactness approach to induction on energy.

Let $s_{c}=\frac{d}{2}-\frac{2}{p-1}$, for the $\dot{H}^{s_{c}}$-critical NLW 3.1 with $p \neq 1+\frac{4}{d-2}$, both the Morawetz estimate and energy conservation fail. It is hard to prove global well posedness and scattering of equation 3.1 in the space $\dot{H}^{s_{c}} \times \dot{H}^{s_{c}-1}$. Up to now, we do not know how to treat the large-data case since there does not exist any a priori control of a critical norm. The first result in this direction is due to KenigMerle [78, where they studied the $\dot{H}^{\frac{1}{2}}$-critical Schrödinger equation in $\mathbb{R}^{3}$. For the defocusing energy-supercritical NLW in odd dimensions, Kenig and Merle [79, 80] 
proved that if the radial solution $u$ is apriorily bounded in the critical Sobolev space, that is

$$
\left(u, u_{t}\right) \in L_{t}^{\infty}\left(I ; \dot{H}_{x}^{s_{c}}\left(\mathbb{R}^{d}\right) \times \dot{H}_{x}^{s_{c}-1}\left(\mathbb{R}^{d}\right)\right), \quad s_{c}:=\frac{d}{2}-\frac{2}{p-1}>1,
$$

then $u$ is global and scatters. Later, Killip and Visan [83] showed the result in $\mathbb{R}^{3}$ for the non-radial solutions by making use of Huygens principle and so called "localized double Duhamel trick". We refer to [10, 84, 110, for some high dimensional cases. Recently, Duyckaerts, Kenig and Merle [40] obtain such result for the focusing energy-supercritical NLW with radial solution in three dimension. Their proof relies on the compactness/rigidity method, pointwise estimates on compact solutions obtained in 79, and the channels of energy method developed in [37. Furthermore, by exploiting the double Duhamel trick, Dodson and Lawrie[29] extend the result in 40 to dimension five.

\subsection{Critical norm conjecture.}

Conjecture 3.1 (Critical norm conjecture). Let $s_{c} \geq 1$ and $\mu=-1$. Suppose $u: I \times \mathbb{R}^{d} \rightarrow \mathbb{R}$ is a maximal-lifespan solution to (3.1) such that

$$
\left(u, \partial_{t} u\right) \in L_{t}^{\infty}\left(I ; \dot{H}_{x}^{s_{c}} \times \dot{H}^{s_{c}-1}\left(\mathbb{R}^{d}\right)\right) .
$$

Then $u$ is global and scatters in the sense that there exist unique $\left(u_{0}^{ \pm}, u_{1}^{ \pm}\right) \in$ $\left.\dot{H}_{x}^{s_{c}}\left(\mathbb{R}^{d}\right) \times \dot{H}^{s_{c}-1}\left(\mathbb{R}^{d}\right)\right)$ such that

$$
\lim _{t \rightarrow \pm \infty}\left\|u(t)-S(t)\left(u_{0}^{ \pm}, u_{1}^{ \pm}\right)\right\|_{\dot{H}_{x}^{s_{c}}\left(\mathbb{R}^{d}\right)}=0 .
$$

Conjecture 3.1 implies that

$$
\limsup _{t \rightarrow T_{+}(u)}\|\vec{u}(t)\|_{\dot{H}^{s_{c}}\left(\mathbb{R}^{3}\right) \times \dot{H}^{s_{c}-1}\left(\mathbb{R}^{3}\right)}<\infty \Longrightarrow u \text { scatters forward in time. }
$$

The well-known results on defocusing Conjecture:

\begin{tabular}{|c|c|}
\hline$d=3$ & $p>4$ \\
\hline radial & Kenig-Merle [79] \\
\hline non-radial & Killip-Visan [83], p-1 even \\
\hline
\end{tabular}

\begin{tabular}{|c|c|c|c|}
\hline$d=4$ & $3<p<5$ & $p=5$ & $p>5$ \\
\hline radial & Killip-Visan[84] & & Miao-Wu-Zhang \\
\hline non-radial & Miao-Wu-Zhang & Miao-Wu-Zhang & Open! \\
\hline
\end{tabular}

\begin{tabular}{|c|c|c|c|c|}
\hline$d=5$ & $7 / 3<p<3$ & $p=3$ & $3<p<5$ & $p \geq 5$ \\
\hline radial & Killip-Visan[84] & & Open! & Kenig-Merle [80] \\
\hline non-radial & Easy & Bulut [10] & Open! & Open! \\
\hline
\end{tabular}

\begin{tabular}{|c|c|c|c|c|}
\hline$d=6$ & $2<p<7 / 3$ & $7 / 3<p<3$ & $p=3$ & $p-1$ even or $p>3$ \\
\hline radial & Killip-Visan[84] & & & \\
\hline non-radial & Easy & Easy & Bulut[10] & Easy \\
\hline
\end{tabular}

\begin{tabular}{|c|c|c|c|}
\hline$d \geq 7$ & $\frac{4}{d-2}<p-1<\frac{d(d-1)-\sqrt{d^{2}(d-1)^{2}-16(d+1)^{2}}}{2(d+1)}$ & $p=3$ & other \\
\hline radial & Killip-Visan[84] & & \\
\hline non-radial & Easy & Bulut[10] & Easy \\
\hline
\end{tabular}


It is OPEN: $d=4,5, p>1+\frac{4}{d-3}$ !

The well-known results on focusing Conjecture:

\begin{tabular}{|c|c|c|}
\hline & radial & nonradial \\
\hline$d=3$ & Duyckaerts-Kenig-Merle [40] & open! \\
\hline$d=5$ & Dodson -Lawrie[29] & open! \\
\hline$d$ even & open & open! \\
\hline
\end{tabular}

3.2. Nonscattering radial solution. Duyckaerts and Roy in [46] proved the existence of nonscattering radial solution to supercritical wave equation.

Theorem 3.2 (Nonscattering radial solution, Duyckaerts-Roy [46]). Assume $d=3$ and $p>5$. Let $u$ be a solution of $u_{t t}-\Delta u \pm|u|^{p-1} u=0$ with radial data $\left(u_{0}, u_{1}\right) \in$ $\dot{H}^{s_{c}}\left(\mathbb{R}^{3}\right) \times \dot{H}^{s_{c}-1}\left(\mathbb{R}^{3}\right)$. Then

- either $T_{+}(u)<+\infty$ and

$$
\lim _{t \rightarrow T_{+}(u)}\left\|\left(u(t), \partial_{t} u(t)\right)\right\|_{\dot{H}^{s_{c}}\left(\mathbb{R}^{3}\right) \times \dot{H}^{s_{c}-1}\left(\mathbb{R}^{3}\right)}=+\infty,
$$

- or $T_{+}(u)=+\infty$ and $u$ scatters forward in time.

An analogous statement holds for negative times.

\section{Question: How about NLS?}

Theorem 3.2 is equivalent to (3.7) with the limit superior replaced by a limit inferior. We do not know any direct application of this qualitative improvement, however its analog in the case $p=5$, is crucial in the proof of the soliton resolution conjecture for the energy-critical wave equation in [39.

Theorem 3.2 means exactly that solutions of $3 \mathrm{~d}$ energy-supercritical NLW are of one of the following three types: scattering solutions, solutions blowing-up in finite time with a critical norm going to infinity at the maximal time of existence, and global solutions with a critical norm going to infinity for infinite times.

In the defocusing case, it is conjectured that all solutions with initial data in the critical space $\dot{H}^{s_{c}}$ scatter. The difficulty of this conjecture is of course the lack of conservation law at the level of crirtical regularity. The only supercritical dispersive equations for which scattering was proved for all solutions are wave and Schrödinger equations with defocusing barely supercritical nonlinearities: see e.g [121, 122, 128, 140, $]^{3}$

In the focusing case, solutions blowing up in finite time are known. One type of finite time blow-up is given by the ODE $y^{\prime \prime}=|y|^{p-1} y$, and is believed to be stable: see [33, 34] for stability inside the wave cone.

3.3. Stability in $H^{2} \times H^{1}$ of the ODE blowup profile. Consider the Cauchy problem for the semilinear wave equation

$$
\partial_{t}^{2} u-\Delta u=|u|^{p-1} u, \quad p>3
$$

for $x \in \mathbb{R}^{3}$.

\footnotetext{
${ }^{3}$ In 121 the scattering with data in $\tilde{H}^{2}:=\dot{H}^{2} \cap \dot{H}^{1}\left(\mathbb{R}^{3}\right)$ is not explicitely mentionned. However, it can be easily derived from the finite bounds of the $L_{t}^{4}\left(\mathbb{R}, L_{x}^{12}\right)$ norm and the $L_{t}^{\infty}\left(\mathbb{R}, \tilde{H}^{2}\right)$ norm of the solution, by using a similar argument to that in 122 to prove scattering.
} 
First, recall that the existence of finite-time blowup is most easily seen by ignoring the Laplacian in (3.9). The remaining ODE in $t$ can be solved explicitly which leads to the solution

$$
u_{1}(t, x)=c_{p}(1-t)^{-\frac{2}{p-1}}, \quad c_{p}=\left(\frac{2(p+1)}{(p-1)^{2}}\right)^{\frac{1}{p-1}} .
$$

Obviously, $u_{1}$ becomes singular at $t=1$. One might object that this solution does not have data in $H^{2} \times H^{1}\left(\mathbb{R}^{3}\right)$. However, this defect is easily fixed by using suitable cut-offs and exploiting finite speed of propagation. By using symmetries of the equation one can in fact produce a much larger family of blowup solutions. For instance, the time translation symmetry immediately yields the one-parameter family

$$
u_{T}(t, x):=c_{p}(T-t)^{-\frac{2}{p-1}}
$$

and by applying the Lorentz transform $\Lambda_{T}(a)$, we can even generate the 4 -parameter family

$$
u_{T, a}(t, x) \triangleq u_{T}\left(\Lambda_{T}(a)(t, x)\right)
$$

of explicit blowup solutions, Lorentz boosts is as follows: for $a=\left(a^{1}, a^{2}, a^{3}\right) \in \mathbb{R}^{3}$ and $T \in \mathbb{R}$, we set

$$
\Lambda_{T}(a)=\Lambda_{T}^{3}\left(a^{3}\right) \Lambda_{T}^{2}\left(a^{2}\right) \Lambda_{T}^{1}\left(a^{1}\right),
$$

where $\Lambda^{j}: \mathbb{R} \times \mathbb{R}^{3} \rightarrow \mathbb{R} \times \mathbb{R}^{3}, j \in\{1,2,3\}$, is defined by

$$
\Lambda_{T}^{j}\left(a^{j}\right):\left\{\begin{array}{l}
t \mapsto(t-T) \cosh a^{j}+x^{j} \sinh a^{j}+T \\
x^{k} \mapsto x^{k}+\delta^{j k}\left[(t-T) \sinh a^{j}+x^{j} \cosh a^{j}-x^{j}\right] .
\end{array}\right.
$$

Merle-Zaag in [[103, 104] [105]] proved the stability of the above family of explicit blowup solutions in the following sense.

Theorem 3.3 (Stability in $H^{2} \times H^{1}$ of the ODE blowup profile, ). Fix $p>3$. There exist constants $M, \delta>0$ such that the following holds. Suppose $(f, g) \in H^{2} \times H^{1}\left(\mathbb{R}^{3}\right)$ satisfy

$$
\left\|(f, g)-u_{1,0}[0]\right\|_{H^{2} \times H^{1}\left(B_{1+\delta}\left(x_{0}\right)\right)} \leq \frac{\delta}{M}
$$

for some $x_{0} \in \mathbb{R}^{3}$. Then $T:=T_{f, g}\left(x_{0}\right) \in[1-\delta, 1+\delta]$ and there exists an $a \in$ $B_{M p \delta}\left(x_{0}\right)$ such that the solution $u: \Gamma\left(T_{f, g}\left(x_{0}\right), x_{0}\right) \rightarrow \mathbb{R}$ of 3.9 with data $u[0]=$ $(f, g)$ satisfies

$$
\left\{\begin{array}{l}
(T-t)^{-s_{p}+2}\left\|u[t]-u_{T, a}[t]\right\|_{\dot{H}^{2} \times \dot{H}^{1}\left(\mathbb{B}_{T-t}^{3}\left(x_{0}\right)\right)} \lesssim(T-t)^{\frac{1}{p-1}} \\
(T-t)^{-s_{p}+1}\left\|u[t]-u_{T, a}[t]\right\|_{\dot{H}^{1} \times L^{2}\left(\mathbb{B}_{T-t}^{3}\left(x_{0}\right)\right)} \lesssim(T-t)^{\frac{1}{p-1}} \\
(T-t)^{-s_{p}}\left\|u(t, \cdot)-u_{T, a}(t, \cdot)\right\|_{L^{2}\left(\mathbb{B}_{T-t}^{3}\left(x_{0}\right)\right)} \lesssim(T-t)^{\frac{1}{p-1}}
\end{array}\right.
$$

for all $t \in[0, T)$, where $s_{p}=\frac{3}{2}-\frac{2}{p-1}$ is the critical Sobolev exponent.

3.4. Type II blowup solutions. Let $d \geq 11$ and let the Joseph-Lundgren exponent be

$$
p_{J L}=1+\frac{4}{d-4-2 \sqrt{d-1}} .
$$

Then for $p>p_{J L}$, the soliton profile admits an asymptotic expansion

$$
Q(r)=\frac{c_{\infty}}{r^{\frac{2}{p-1}}}+\frac{a_{1}}{r^{\gamma}}+o\left(\frac{1}{r^{\gamma}}\right), a_{1} \neq 0, \quad \Delta Q+Q^{p}=0,
$$


with

$$
c_{\infty}=\left[\frac{2}{p-1}\left(d-2-\frac{2}{p-1}\right)\right]^{\frac{1}{p-1}}, \quad \gamma=\frac{1}{2}(d-2-\sqrt{\triangle})>0
$$

and where

$$
\triangle=(d-2)^{2}-4 p c_{\infty}^{p-1}\left(\triangle>0 \text { for } p>p_{J L}\right) .
$$

These numbers describing the asymptotic behavior of the soliton are essential in the analysis of type II blow up bubbles as follows:

Theorem 3.4 (Type II blow up for the energy super critical wave equation, [22]). Let $d \geq 11, p_{J L}$ be given by (3.10) and a nonlinearity

$$
p=2 q+1, \quad q \in \mathbb{N}^{*}, \quad p>p_{J L} .
$$

Let $\gamma$ be given by 3.12 and define:

$$
\alpha=\gamma-\frac{2}{p-1} .
$$

Assume moreover:

$$
\left(\frac{d}{2}-\gamma\right) \notin \mathbb{N}
$$

Pick an integer

$$
\ell \in \mathbb{N} \text { with } \ell>\alpha,
$$

and a large enough regularity exponent

$$
s_{+} \in \mathbb{N}, \quad s_{+} \geq s(\ell) \quad(s(\ell) \rightarrow+\infty \text { as } \ell \rightarrow+\infty) .
$$

Then there exists a radially symmetric initial data $\left(u_{0}, u_{1}\right) \in H^{s_{+}} \times H^{s_{+}-1}\left(\mathbb{R}^{d}\right)$ such that the corresponding solution to (NLW) blows up in finite time $0<T<+\infty$ by concentrating the soliton profile:

$$
u(t, r)=\frac{1}{l(t)^{\frac{2}{p-1}}}(Q+\varepsilon)\left(\frac{r}{l(t)}\right)
$$

with:

(i) Blow up speed:

$$
l(t)=c\left(u_{0}\right)\left(1+o_{t \uparrow T}(1)\right)(T-t)^{\frac{\ell}{\alpha}}, \quad c\left(u_{0}\right)>0 ;
$$

(iii) Asymptotic stability above scaling in renormalized variables:

$$
\lim _{t \uparrow T}\left\|\varepsilon(t, \cdot), \lambda\left(\partial_{t} u\right)_{\lambda}(t, \cdot)\right\|_{\dot{H}^{s} \times \dot{H}^{s-1}}=0 \text { for all } s_{c}<s \leq s_{+} ;
$$

(iv) Boundedness below scaling:

$$
\limsup _{t \uparrow T}\left\|u(t), \partial_{t} u(t)\right\|_{\dot{H}^{s} \times \dot{H}^{s-1}}<+\infty \text { for all } 1 \leq s<s_{c} ;
$$

(v) Behavior of the critical norms:

$$
\begin{gathered}
\|u(t)\|_{\dot{H}^{s_{c}}}=\left[c(d, p) \sqrt{\ell}+o_{t \uparrow T}(1)\right] \sqrt{|\log (T-t)|}, \\
\limsup _{t \uparrow T}\left\|\partial_{t} u(t)\right\|_{\dot{H}^{s_{c}-1}}<+\infty .
\end{gathered}
$$

The proof of Theorem 3.4 relies on an explicit construction of blow up solutions. It allows us to find a whole set of initial data leading to such a blow up, and to investigate its topological properties: 
Theorem 3.5. We keep the notations and assumptions of Theorem 3.4. Let a slightly supercritical regularity exponent $\sigma=\sigma(\ell)$ satisfying:

$$
0<\sigma-s_{c} \ll 1 \text { small enough. }
$$

There exists a locally Lipschitz manifold of codimension $\ell-1$ in the Banach space $\dot{H}^{\sigma} \cap \dot{H}^{s_{+}} \times \dot{H}^{\sigma-1} \cap \dot{H}^{s_{+}-1}$ of initial data leading to the blow up scenario described by Theorem 3.4. We point out that as $\alpha>2$, the codimension satisfies $\ell-1>2$.

\section{ENERGY-SUBCRITICAL NLW}

Consider energy subcritical NLW equation

$$
(\mathrm{NLW}) \quad\left\{\begin{array}{l}
\partial_{t t} u-\Delta u=\mu|u|^{p-1} u, \quad(t, x) \in \mathbb{R} \times \mathbb{R}^{d}, \\
\left(u, \partial_{t} u\right)(0, x)=\left(u_{0}, u_{1}\right)(x),
\end{array}\right.
$$

where $u: \mathbb{R}_{t} \times \mathbb{R}_{x}^{d} \rightarrow \mathbb{R}, s_{c}=\frac{d}{2}-\frac{2}{p-1}<1$.

History of defocusing energy-subcritical NLW: $\mu=-1, s_{c}<1$.

(1) For $d=3$ and $1<p<p_{c}=5$, Jörgen in 1961 proved the global existence of smoothing solution of [4.1] [70].

(2) For higher dimensions with $4 \leq d \leq 9$, Brenner, Wahl and Pecher proved the global existence of smoothing solution of (4.1) 8, 114, 144.

(3) Ginibre-Velo proved the global well posedness of energy solution of (4.1) with intial data $\left(u_{0}, u_{1}\right) \in H^{1} \times L^{2}$ [55, 56].

4.1. Critical norm conjecture. The methods employed in the energy-supercritical NLW also lead to the study of the energy-subcritical NLW. In fact, using the channels of energy method pioneered in [37, 39, Shen [127] proved the analog result of 79. for $2<p<4$ with $d=3$ in both defocusing and focusing case. However, the channels of energy method does not work so effectively for $p \leq 2$. Recently, by virial based rigidity argument and improving addition regularity for the minimal counterexamples, Dodson and Lawrie [28] extended the result of [127] to $\sqrt{2}<p \leq 2$. How to establish monotonicity formulae at a different regularity matching with the critical conservation laws is a difficulty intrinsic to the nonlinear wave equation. In order to utilize the monotonicity formulae, one needs to improve the regularity for the almost periodic solutions. In [28, 127, they used the double Duhamel trick to show that almost periodic solutions belong to energy space $\dot{H}_{x}^{1}\left(\mathbb{R}^{3}\right) \times L_{x}^{2}\left(\mathbb{R}^{3}\right)$. The main difficult is that the decay rate of the linear solution is not enough to guarantee the double Duhamel formulae converges. However, the weighted decay available from radial Sobolev embedding can supply the additional decay to guarantee the double Duhamel formulae converges. Thus, one need the radial assumption in 28, 127. This is different from cubic Schrödinger equation [78, where no radial assumption is made. This is due to the Lin-Strauss Morawetz inequality

$$
\int_{I} \int_{\mathbb{R}^{3}} \frac{|u(t, x)|^{4}}{|x|} d x d t \lesssim \sup _{t \in I}\|u(t, x)\|_{\dot{H}_{x}^{\frac{1}{2}}\left(\mathbb{R}^{3}\right)}^{2},
$$

which is scaling critical with the cubic Schrödinger equation.

Conjecture 4.1 (Critical norm conjecture). Let $s_{c}>0$ and $\mu=-1$. Suppose $u: I \times \mathbb{R}^{d} \rightarrow \mathbb{R}$ is a maximal-lifespan solution to (4.1) such that

$$
\left(u, \partial_{t} u\right) \in L_{t}^{\infty}\left(I ; \dot{H}_{x}^{s_{c}} \times \dot{H}^{s_{c}-1}\right) .
$$


Then $u$ is global and scatters in the sense that there exist unique $\left(u_{0}^{ \pm}, u_{1}^{ \pm}\right) \in$ $\dot{H}_{x}^{s_{c}}\left(\mathbb{R}^{d}\right) \times \dot{H}_{x}^{s_{c}-1}\left(\mathbb{R}^{d}\right)$ such that

$$
\lim _{t \rightarrow \pm \infty}\left\|u(t)-S(t)\left(u_{0}^{ \pm}, u_{1}^{ \pm}\right)\right\|_{\dot{H}_{x}^{s_{c}}\left(\mathbb{R}^{d}\right)}=0 .
$$

Defocusing/focusing

\begin{tabular}{|l|c|c|}
\hline & $\sqrt{2}<p \leq 2$ & $2<p<4$ \\
\hline$d=3$ & Dodson-Lawrie [28] & Shen [127] \\
\hline$d \geq 4$ & & \\
\hline
\end{tabular}

\section{The clue for the proof in [28])}

The proof follows the concentration-compactness approach to induction on energy. We argue by contradiction. The failure of scattering result would imply the existence of very special class of solutions. On the other hand, these solutions have so many good properties that they do not exist. Thus we get a contradiction. While we will make some further reductions later, the main property of the special counterexamples is almost periodicity modulo symmetries:

Step 1 Reduction to almost periodic solution

Theorem 4.2 (Reduction to two enemies). Suppose that Conjecture 4.1 for $\dot{H}^{\frac{1}{2}}$ critical wave equation fails, then there exists a minimal $\dot{H}^{\frac{1}{2}}$-energy, maximal-lifespan solution $u: I \times \mathbb{R}^{3} \rightarrow \mathbb{R}$ to 4.1, which is almost periodic modulo symmetries, $\|u\|_{L_{t, x}^{4}\left(I \times \mathbb{R}^{3}\right)}=+\infty$. The almost periodic modulo symmetries means: for $\forall \eta>0$, there exist $C(\eta)>0$ and $N(t)$ defined in I such that

$$
\left\{\begin{array}{l}
\left.\left.\int_{|x| \geq \frac{C(\eta)}{N(t)}}|| \nabla\right|^{\frac{1}{2}} u\right|^{2} d x+\int_{|\xi| \geq C(\eta) N(t)}|\xi| \cdot|\hat{u}(t, \xi)|^{2} d \xi<\eta \\
\left.\left.\int_{|x| \geq \frac{C(\eta)}{N(t)}}|| \nabla\right|^{-\frac{1}{2}} u_{t}\right|^{2} d x+\int_{|\xi| \geq C(\eta) N(t)}|\xi|^{-1} \cdot\left|\hat{u}_{t}(t, \xi)\right|^{2} d \xi<\eta .
\end{array}\right.
$$

Moreover,

(i) $N(t) \equiv 1, \quad \forall t \geq 0 . \quad$ (soliton-like solution)

(ii) $N(t) \leq 1$ and $\varlimsup_{t \rightarrow+\infty} N(t)=0 . \quad$ (Energy-cascade).

The properties of almost periodic solution:

Lemma 4.3 (No-waste Duhamel formula). Let $u:\left(T_{-}, T_{+}\right) \times \mathbb{R}^{3} \rightarrow \mathbb{R}$ be an almost periodic solution, then

$$
\vec{u}(t) \triangleq\left(u, u_{t}\right)(t)=\lim _{T \rightarrow T_{ \pm}} \int_{t}^{T} S(t-s)\left(0, \pm|u|^{2} u\right)(s) d s,
$$

where the limit is to be understood in the weak $L^{2}$ topology.

Proposition 4.4 (Negative energy implies that the finite time blow-up). Let $u:\left(T_{-}, T_{+}\right) \times \mathbb{R}^{3} \rightarrow \mathbb{R}$ be the maximal-lifespan solution for $3 d$ focuing cubic wave equation with $E\left(u_{0}, u_{1}\right) \leq 0$. Then either $u \equiv 0$, or $u$ blows up both forward and backward in finite time (i.e. $T_{-}>-\infty$ and $\left.T_{+}<\infty\right)$.

Step 2. Additional regularity and preclude energy-critical cascade. 
Theorem 4.5 (Additional regularity). Let $T_{-}<0$, and $u:\left(T_{-},+\infty\right) \times \mathbb{R}^{3} \rightarrow \mathbb{R}$ be an almost periodic solution with $N(t) \leq 1$, then $\vec{u}(t) \in \dot{H}^{1} \times L^{2}$ with

$$
\|\vec{u}(t)\|_{\dot{H}^{1} \times L^{2}} \lesssim N(t)^{\frac{1}{2}} .
$$

Remark 4.6. Exponent $1 / 2$ is fully determined by scaling invariant of (NLW). In fact,

$$
\frac{u}{(\Delta t)^{2}} \simeq u^{3} \Longrightarrow u \simeq(\Delta t)^{-1} \simeq N(t)
$$

hence

$$
\int_{\mathbb{R}^{3}}|\nabla u|^{2} d x \simeq \frac{|u|^{2}}{(\Delta x)^{2}}(\Delta x)^{3} \simeq|u|^{2}(\Delta x) \simeq N(t) .
$$

To preclude energy-cascade solution: By making use of Theorem 4.5 and Sobolev embedding theorem, we have

$$
\left\{\begin{array}{l}
\varlimsup_{t \rightarrow \infty}\|\vec{u}(t)\|_{\dot{H}^{1} \times L^{2}} \lesssim \varlimsup_{t \rightarrow \infty} N(t)^{\frac{1}{2}}=0, \\
\|u(t)\|_{L^{4}}^{2} \lesssim\|u\|_{\dot{H}^{\frac{3}{4}}}^{2} \lesssim\|u\|_{\dot{H}^{\frac{1}{2}}}\|u\|_{\dot{H}^{1}} \rightarrow 0,
\end{array} \quad \Longrightarrow E\left(u_{0}, u_{1}\right)=0\right.
$$

This fact together with Proposition 4.4 precludes the energy-cascade solution. Therefore, to remove energy-cascade solution can be reduced to Theorem 4.5

Step 3. Additional compactness and to preclude quasi-soliton solution.

Due to $N(t) \equiv 1$, this almost periodic solution enjoys compactness modulo symmetries.

Proposition 4.7 (Compactness and more additional regularity). Let $u: \mathbb{R} \times \mathbb{R}^{3} \rightarrow$ $\mathbb{R}$ be a almost periodic solution with $N(t) \equiv 1$, then

$$
K \triangleq\{\vec{u}(t): t \in \mathbb{R}\}
$$

is a precompact in $\left(\dot{H}^{\frac{1}{2}} \times \dot{H}^{-\frac{1}{2}}\right) \cap\left(\dot{H}^{1} \times L^{2}\right)$ This fact can be reduced to prove $\vec{u} \in \dot{H}^{1+} \times \dot{H}^{0+}$.

Lemma 4.8. Let $\vec{u} \in \dot{H}^{1} \times L^{2}$ be the solution for 3d cubic wave equation, then for any $R>0$,

$$
\begin{aligned}
& \frac{d}{d t}\left\langle u_{t}, \chi_{R}\left(u+r u_{r}\right)\right\rangle \\
= & -\frac{1}{\left|S^{2}\right|} E(\vec{u})(t)+\int_{0}^{\infty}\left(1-\chi_{R}\right)\left(\frac{1}{2} u_{t}^{2}+\frac{1}{2} u_{r}^{2} \mp \frac{1}{4} u^{4}\right) r^{2} d r \\
& -\int_{0}^{\infty}\left(\frac{1}{2} u_{t}^{2}+\frac{1}{2} u_{r}^{2} \pm \frac{1}{4} u^{4}\right) r \chi_{R}^{\prime} r^{2} d r-\int_{0}^{\infty} u u_{r} r \chi_{R}^{\prime} r d r,
\end{aligned}
$$

where

$$
\langle f, g\rangle \triangleq \int_{0}^{\infty} f(r) g(r) r^{2} d r, \quad \chi_{R}(r)=\chi(r / R), \quad \chi \in C_{c}^{\infty}(\mathbb{R}) .
$$

Proof.

$$
\begin{aligned}
\frac{d}{d t}\left\langle u_{t}, \chi_{R}\left(u+r u_{r}\right)\right\rangle & =\int u_{t t} \chi_{R}\left(u+r u_{r}\right) r^{2} d r+\int u_{t} \chi_{R}\left(u_{t}+r \partial_{r} u_{t}\right) r^{2} d r \\
& \triangleq I_{1}+I_{2} .
\end{aligned}
$$




\section{To calculate $I_{2}$ :}

$$
\begin{aligned}
I_{2} & =\int\left|u_{t}\right|^{2} \chi_{R} r^{2} d r+\int u_{t} \partial_{r} u_{t} \chi_{R} r^{3} d r \\
& =\int\left|u_{t}\right|^{2} \chi_{R} r^{2} d r-\frac{1}{2} \int\left|u_{t}\right|^{2} \partial_{r}\left(\chi_{R} r^{3}\right) d r \\
& =-\frac{1}{2} \int\left|u_{t}\right|^{2} \chi_{R} r^{2} d r-\frac{1}{2} \int\left|u_{t}\right|^{2} \chi_{R}^{\prime} r^{3} d r .
\end{aligned}
$$

To calculate $I_{1}$ : Making use of

$$
u_{t t}=\Delta u \pm u^{3}=\partial_{r}^{2} u+2 r^{-1} \partial_{r} u \pm u^{3},
$$

and taking it into $I_{1}$, one easily verifies that

$$
\begin{aligned}
I_{1} & =\int \partial_{r}^{2} u \chi_{R}\left(u+r u_{r}\right) r^{2} d r+2 \int \partial_{r} u \chi_{R}\left(u+r u_{r}\right) r d r \pm \int u^{3} \chi_{R}\left(u+r u_{r}\right) r^{2} d r \\
& \triangleq I_{11}+I_{12} \pm I_{13} .
\end{aligned}
$$

\section{To calculate $I_{13}$ :}

$$
\begin{aligned}
I_{13} & =\int \chi_{R}|u|^{4} r^{2} d r+\int u^{3} u_{r} \chi_{R} r^{3} d r \\
& =\int \chi_{R}|u|^{4} r^{2} d r-\frac{1}{4} \int|u|^{4} \partial_{r}\left(\chi_{R} r^{3}\right) d r \\
& =\frac{1}{4} \int \chi_{R}|u|^{4} r^{2} d r-\frac{1}{4} \int|u|^{4} \chi_{R}^{\prime} r^{3} d r .
\end{aligned}
$$

To calculate $I_{11}+I_{12}$ :

$$
\begin{aligned}
I_{11} & =\int \partial_{r}^{2} u u \chi_{R} r^{2} d r+\int \partial_{r}^{2} u u_{r} \chi_{R} r^{3} d r \\
& =-\int\left|u_{r}\right|^{2} \chi_{R} r^{2} d r-\int u_{r} u \partial_{r}\left(\chi_{R} r^{2}\right) d r-\frac{1}{2} \int\left|u_{r}\right|^{2}\left(\chi_{R} r^{3}\right)_{r} d r \\
& =-\frac{5}{2} \int\left|u_{r}\right|^{2} \chi_{R} r^{2} d r+\frac{1}{2} \int|u|^{2} \partial_{r}^{2}\left(\chi_{R} r^{2}\right) d r-\frac{1}{2} \int\left|u_{r}\right|^{2} \chi_{R}^{\prime} r^{3} d r \\
I_{12} & =2 \int \partial_{r} u u \chi_{R} r d r+2 \int\left|u_{r}\right|^{2} \chi_{R} r^{2} d r \\
& =-\int|u|^{2}\left(\chi_{R} r\right)_{r} d r+2 \int\left|u_{r}\right|^{2} \chi_{R} r^{2} d r, \\
I_{11}+I_{12} & =-\frac{1}{2} \int\left|u_{r}\right|^{2} \chi_{R} r^{2} d r+\frac{1}{2} \int|u|^{2} \partial_{r}\left(\chi_{R}^{\prime} r^{2}\right) d r-\frac{1}{2} \int\left|u_{r}\right|^{2} \chi_{R}^{\prime} r^{3} d r \\
& =-\frac{1}{2} \int\left|u_{r}\right|^{2} \chi_{R} r^{2} d r-\int u u_{r} \chi_{R}^{\prime} r^{2} d r-\frac{1}{2} \int\left|u_{r}\right|^{2} \chi_{R}^{\prime} r^{3} d r .
\end{aligned}
$$


Hence,

$$
\begin{aligned}
& \frac{d}{d t}\left\langle u_{t}, \chi_{R}\left(u+r u_{r}\right)\right\rangle \\
= & -\int\left[\frac{1}{2}\left|u_{r}\right|^{2}+\frac{1}{2}\left|u_{t}\right|^{2} \mp \frac{1}{4}|u|^{4}\right] \chi_{R} r^{2} d r \\
& -\int\left[\frac{1}{2}\left|u_{r}\right|^{2}+\frac{1}{2}\left|u_{t}\right|^{2} \pm \frac{1}{4}|u|^{4}\right] \chi_{R}^{\prime} r^{3} d r-\int u u_{r} \partial_{r} \chi_{R}^{\prime} r^{2} d r \\
= & -\frac{1}{\left|S^{2}\right|} E(\vec{u})+\int\left(1-\chi_{R}\right)\left[\frac{1}{2}\left|u_{r}\right|^{2}+\frac{1}{2}\left|u_{t}\right|^{2} \mp \frac{1}{4}|u|^{4}\right] r^{2} d r \\
& -\int\left[\frac{1}{2}\left|u_{r}\right|^{2}+\frac{1}{2}\left|u_{t}\right|^{2} \pm \frac{1}{4}|u|^{4}\right] \chi_{R}^{\prime} r^{3} d r-\int u u_{r} \chi_{R}^{\prime} r^{2} d r .
\end{aligned}
$$

\section{To preclude quasi-soliton solution:}

$$
\text { Claim : } \forall \eta>0, E(\vec{u}) \leq C \eta \text {. }
$$

This together with Propsition 4.4 can kill the quasi-soliton solution.

Step 4. We shall use Propositin 4.7 and Lemma 4.8 to prove the above Claim.

First, compactness implies that for $\forall \eta>0$, there exists $R_{0}=R_{0}(\eta)$ such that when $R \geq R_{0}(\eta)$,

$$
\left\{\begin{array}{l}
\int_{R}^{\infty}\left[u_{t}^{2}+u_{r}^{2}\right](t) r^{2} d r<\eta, \\
\dot{H}^{\frac{1}{2}} \cap \dot{H}^{1} \hookrightarrow \dot{H}^{\frac{3}{4}} \hookrightarrow L^{4}
\end{array} \Longrightarrow \int_{R}^{\infty} u^{4}(t) r^{2} d r<\eta, \quad \forall t \in \mathbb{R}\right.
$$

Note that

$$
\int_{R}^{\infty} f(r)^{2} d r+R f(R)^{2}=-\int_{R}^{\infty} f_{r}(r) f(r) r d r, f \in \dot{H}^{1}
$$

it follows that

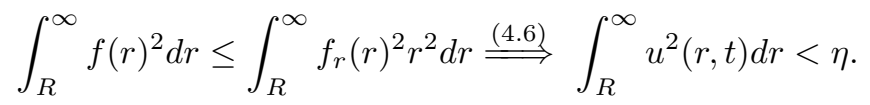

In fact, it suffice to prove

$$
\int_{0}^{\infty} u^{2} d r \lesssim \int_{0}^{\infty}\left|\frac{u}{r}\right|^{2} r^{2} d r \lesssim\|u\|_{\dot{H}^{1}}^{2}<\infty,
$$

by the Hardy inequality. This further implies that

$$
\left\{\begin{array}{l}
\left|\int_{0}^{\infty}\left(1-\chi_{R}\right)\left(\frac{1}{2} u_{t}^{2}+\frac{1}{2} u_{r}^{2} \pm \frac{1}{4} u^{4}\right) r^{2} d r\right| \leq C \eta \\
\left|\int_{0}^{\infty}\left(\frac{1}{2} u_{t}^{2}+\frac{1}{2} u_{r}^{2} \pm \frac{1}{4} u^{4}\right) r \chi_{R}^{\prime} r^{2} d r\right| \lesssim C \eta \\
\left|\int_{0}^{\infty} u u_{r} r \chi_{R}^{\prime} r d r\right| \lesssim C \eta
\end{array}\right.
$$


Therefore, we integrate 4.5 with respect to $r$ from 0 to $T$ to see

$$
\begin{aligned}
E(\vec{u}) \leq & C \eta+\frac{1}{T}\left|\left\langle u_{t}(t), \chi_{R}\left(u+r u_{r}\right)(t)\right\rangle\right|_{0}^{T} \\
\leq & C \eta+\frac{C}{T} \int_{0}^{T}\left|u_{t}(T)\right| \cdot|u(T)| r^{2} d r+\frac{C}{T} \int_{0}^{T}\left|u_{t}(0)\right| \cdot|u(0)| r^{2} d r \\
& +\frac{C}{T} \int_{0}^{T}\left|u_{t}(T)\right| \cdot\left|u_{r}(T)\right| r^{3} d r+\frac{C}{T} \int_{0}^{T}\left|u_{t}(0)\right| \cdot\left|u_{r}(0)\right| r^{3} d r
\end{aligned}
$$

where we choose $R=T$ with $T \gg R_{0}(\eta)$.

on the other hand,

$$
\begin{aligned}
\frac{1}{T} \int_{0}^{T}\left|u_{t}\right| \cdot|u| r^{2} d r & \leq \frac{1}{T}\left(\int_{0}^{T}\left|u_{t}\right|^{2} r^{2} d r\right)^{\frac{1}{2}}\left(\int_{0}^{T}|u|^{3} r^{2} d r\right)^{\frac{1}{3}}\left(\int_{0}^{T} r^{2} d r\right)^{\frac{1}{6}} \\
& \leq \frac{C}{T^{\frac{1}{2}}}\left\|u_{t}\right\|_{2}\|u\|_{\dot{H}^{\frac{1}{2}}}, \\
\frac{1}{T} \int_{0}^{T}\left|u_{t}\right| \cdot\left|u_{r}\right| r^{3} d r & \leq \frac{R(\eta)}{T} \int_{0}^{R(\eta)}\left|u_{t}\right| \cdot\left|u_{r}\right| r^{2} d r+\frac{1}{T} \int_{R(\eta)}^{T}\left|u_{t}\right| \cdot\left|u_{r}\right| r^{3} d r \\
& \leq \frac{R(\eta)}{T}\left\|u_{t}\right\|_{2}\|u\|_{\dot{H}^{1}}+\left(\int_{R(\eta)}^{\infty} u_{t}^{2} r^{2} d r\right)^{\frac{1}{2}}\left(\int_{R(\eta)}^{\infty} u_{r}^{2} r^{2} d r\right)^{\frac{1}{2}} \\
& \leq \frac{R(\eta)}{T}+\eta .
\end{aligned}
$$

Letting $T \rightarrow+\infty$, we prove the Claim, this completes the Theorem.

In conclusion From the above induction, we only need to prove Theorem 4.5 and Proposition 4.7.

\subsection{Low regularity.}

Conjecture 4.9 (Low regularity conjecture). Let $s_{c} \geq 0, \mu=-1$ and $\left(u_{0}, u_{1}\right) \in$ $\dot{H}^{s_{c}} \times \dot{H}^{s_{c}-1}$. Then $u$ is global and scatters in the sense that there exist unique $\left(u_{0}^{ \pm}, u_{1}^{ \pm}\right) \in \dot{H}_{x}^{s_{c}}\left(\mathbb{R}^{d}\right) \times \dot{H}^{s_{c}-1}$ such that

$$
\lim _{t \rightarrow \pm \infty}\left\|u(t)-S(t)\left(u_{0}^{ \pm}, u_{1}^{ \pm}\right)\right\|_{\dot{H}_{x}^{s_{c}}\left(\mathbb{R}^{d}\right)}=0 .
$$

Low regularity problem, where we ask what is the minimal $s$ to ensure that (NLW) has either a local solution or a global solution for which the scattering hold?

(i) Miao-Zhang [109] studied the Cauchy problem for the semilinear wave equation

$$
\left\{\begin{array}{l}
u_{t t}-\triangle u=-|u|^{\rho-1} u, \quad(t, x) \in \mathbb{R} \times \mathbb{R}^{d}, \quad d \geq 3, \\
u(x, 0)=\phi(x) \in\left(\dot{H}^{s} \cap L^{\rho+1}\right)\left(\mathbb{R}^{d}\right), \quad u_{t}(x, 0)=\psi(x) \in \dot{H}^{s-1}\left(\mathbb{R}^{d}\right),
\end{array}\right.
$$

and proved the global well-posedness of low regularity solution for 4.9 in $\dot{H}^{s} \times$ $\dot{H}^{s-1}$ with $s \geq \alpha(\rho)$, where

$$
\alpha(\rho) \triangleq\left\{\begin{array}{l}
\frac{2(\rho-1)^{2}-[d+2-\rho(d-2)][d+1-\rho(d-1)]}{2(\rho-1)[d+1-\rho(d-3)]}, \\
k_{0}(d) \leq \rho<\frac{d-1}{d-3}, k_{0}(d)=\frac{(d+1)^{2}}{(d-1)^{2}+4}, \quad d \geq 3
\end{array}\right.
$$




$$
\begin{gathered}
\alpha(\rho) \triangleq\left\{\begin{array}{l}
\frac{4(\rho-1)+[d+2-\rho(d-2)](d \rho-d-4)}{2(\rho-1)[d+4-\rho(d-2)]} \\
\frac{d-1}{d-3} \leq \rho<\frac{d+2}{d-2}, \quad d=5
\end{array}\right. \\
\alpha(\rho) \triangleq\left\{\begin{array}{l}
\frac{2 \rho(\rho-1)+[d+2-\rho(d-2)](d \rho-d-\rho-2)}{2(\rho-1)[d+2-\rho(d-3)]} \\
\frac{d-1}{d-3} \leq \rho<\min \left(\frac{d+2}{d-2}, \frac{d}{d-3}\right), \quad d \geq 6 .
\end{array}\right.
\end{gathered}
$$

(ii) In [126, Shen proved that for $d=3$, and

$$
p \in\left(\frac{11}{3}, 5\right), s_{0}(p)=\frac{2+(5-p) s_{c}}{7-p} \in\left(s_{c}, 1\right),
$$

then for $\left(u_{0}, u_{1}\right) \in\left(\dot{H}^{s_{c}} \cap \dot{H}^{s}\right) \times\left(\dot{H}^{s_{c}-1} \cap \dot{H}^{s-1}\right)$, the solution $u$ to $u_{t t}-\Delta u+$ $|u|^{p-1} u=0$ with initial data $\left(u_{0}, u_{1}\right)$ is global.

(iii) The most interesting problem is $3 \mathrm{~d}$ cubic wave equation

$$
\left\{\begin{array}{l}
u_{t t}-\Delta u=-u^{3}, \quad(t, x) \in \mathbb{R} \times \mathbb{R}^{3}, \\
\left(u, \partial_{t} u\right)(0)=\left(u_{0}, u_{1}\right), \quad x \in \mathbb{R}^{3} .
\end{array}\right.
$$

The well-known results on the cubic wave equation in $\mathbb{R}^{3}$

\begin{tabular}{|c|c|c|}
\hline$s>3 / 4$ & $s=3 / 4$ & $s>\frac{7}{10}$ or $\frac{13}{18}$ \\
\hline KPV[82], Gallagher-Planchon [49] & Bahouri-Chemin [2] & Roy [119], [120] \\
\hline
\end{tabular}

Theorem 4.10 (Dodson 26] ). Suppose there exists a positive constant $\varepsilon>0$ such that

$$
\left\|u_{0}\right\|_{\dot{H}^{1 / 2+\varepsilon}\left(\mathbf{R}^{3}\right)}+\left\||x|^{2 \varepsilon} u_{0}\right\|_{\dot{H}^{1 / 2+\varepsilon}\left(\mathbf{R}^{3}\right)} \leq A<\infty,
$$

and

$$
\left\|u_{1}\right\|_{\dot{H}^{-1 / 2+\varepsilon}\left(\mathbf{R}^{3}\right)}+\left\||x|^{2 \varepsilon} u_{1}\right\|_{\dot{H}^{-1 / 2+\varepsilon}\left(\mathbf{R}^{3}\right)} \leq A<\infty .
$$

Then 4.10) has a global solution and there exists some $C(A, \varepsilon)<\infty$ such that

$$
\int_{\mathbf{R}} \int(u(t, x))^{4} d x d t \leq C(A, \varepsilon)
$$

which proves that $u$ scatters both forward and backward in time.

Theorem 4.11 (Dodson [27] ). The Cauchy problem (4.10) is globally well-posed and scattering for radial initial data $\left.\left(u_{0}, u_{1}\right) \in \dot{B}_{1,1}^{2}\left(\mathbf{R}^{3}\right) \times \in \dot{B}_{1,1}^{1}\left(\mathbf{R}^{3}\right)\right)$. Moreover,

$$
\|u\|_{L_{t, x}^{4}\left(\mathbf{R} \times \mathbf{R}^{3}\right)} \leq C\left(\left\|u_{0}\right\|_{\dot{B}_{1,1}^{2}},\left\|u_{1}\right\|_{\dot{B}_{1,1}^{1}}\right) .
$$

This is the first result in which large data scattering was proved for initial data in a scale - invariant space for which the norm was not controlled by a conserved quantity. 
4.3. Description of local singularity for type I blow up bubble. From finite speed of propagation, using the constant in space solution

$$
u(t, x)=\frac{C}{(T-t)^{\frac{2}{p-1}}}
$$

one can construct solutions blowing up like the ODE $u_{t t}=u^{p}$. They are called type I blow up and correspond to a complete blow up

$$
\lim _{t \rightarrow T}\left\|u(t), \partial_{t} u(t)\right\|_{\dot{H}^{1} \times L^{2}} \rightarrow+\infty .
$$

In the subconformal case $\left(s_{c} \leq \frac{1}{2}\right)$ the recent works by Merle and Zaag [103, 104, 105. give in particular a complete description of the local singularity, being always a type I blow up bubble, and we refer to this monumental series of works for complete references on the history of the problem. Recently also, general upper bounds on the blow up rates have been obtained [61, [83] in the superconformal, energy subcritical case $\left(\frac{1}{2}<s_{c}<1\right)$.

\section{Nonlinear Klein-Gordon equation}

Let $\square=\partial_{t t}-\Delta$, we consider

$$
\square u+u+f(u)=0, \quad(\mathrm{NLKG}) .
$$

Assumptions:

(1) Structure condition: $F$ satisfies $\frac{\partial F(z)}{\partial \bar{z}}=f(z), F(0)=0$.

(2) Smooth and growth conditions: For $\frac{4}{d}<p_{1}<p_{2}<\frac{4}{d-2}$

$$
|f(u)-f(v)| \leq C\left(|u|^{p_{1}}+|v|^{p_{1}}+|u|^{p_{2}}+|v|^{p_{2}}\right)|u-v|, \quad f(0)=0 .
$$

(3) Repulsive condition. If $V(u)=\frac{F(u)}{|u|^{2}}: \mathbb{C} \rightarrow \mathbb{R}$ then

$$
\partial_{|z|} V(z)=2 \operatorname{Re}\left[\partial_{z} V(z) \frac{z}{|z|}\right] \geq 0,
$$

which means that $V(u)$ is non-decreasing function on $|u|$.

\section{The classical Morawetz estimate}

$$
\iint_{\mathbb{R}^{d+1}} \frac{G(u)}{|x|} d x d t \leq C E(u),
$$

where

$$
G(z)=\operatorname{Re}\left(\partial_{z} V(z)|z|^{2} z\right)=\operatorname{Re}(\bar{z} f(z))-F(z) .
$$

Remark 5.1. (i) As we know, the scattering theory for defocuing (NLKG) is based on the classical Morawetz estimate (5.2), from which we can deduce the decay estimate of the solution. This ensures the potential energy has no concentration.

(ii) The introduction of $V(z)$ makes us to compare (NLKG) with the following linear equation

$$
\square u+u+V(x) u=0 .
$$

Berestyeki-Lions have shown that the above equations have the traveling wave solution under the condition $V(z)<V(0)$, which shows that the wave operator is not surjection. The current study is constrained under

$$
\partial_{|z|} V(z) \geq C \min \left(|z|^{-1},|z|^{\gamma}\right), \quad \gamma>0
$$


i.e., $V(u)$ is not flat at $u=0$, and diverse at $u=\infty$. As for the general case

$$
V(z) \geq V(0), \quad \forall z \in \mathbb{C}
$$

it is still an open problem whether one can establish the scattering theory of (NLKG).

\section{Generalized Morawetz inequality}

Under the repulsive condition, Nakanishi derived the modified Morawetz estimate independent of the form of nonlinearity by time-space multiplier argument, which is suitable for different dimensions(including $d=1,2$ )! The arguments are based on

(1) Generalized multiplier $(h \cdot \mathcal{D} u+q u)$.

(2) The Hardy inequality and generalized Gagliardo-Nirenberg inequality

$$
\int_{\mathbb{R}^{d}} \kappa^{2}|u|^{p} d x \leq\|u\|_{q}^{p-2} \int_{\mathbb{R}^{d}}\left(\kappa^{2}|\nabla u+i \lambda u|^{2}+|u \nabla \kappa|^{2}\right) d x,
$$

where $p>2, q=\frac{d(p-2)}{2}$.

Assume that $f(u)$ satisfies the structure, growth and repulsive conditions, and

$$
2+\frac{4}{d}<p<\infty, \quad d \leq 2 ; \quad \text { or } \quad 2+\frac{4}{d}<p<2+\frac{4}{d-2}, d \geq 3
$$

then the energy solution $u$ of (NLKG) satisfies that

$$
\iint_{|x| \leq t} \frac{|u|^{p}}{|t|} d x d t \leq C E(u)^{\frac{p}{2}}
$$

where $C=C(d, p)$ is independent of the growth exponents $p_{1}$ or $p_{2}$ in nonlinearity.

The modified Morawetz estimate breaks through the restriction on dimension. For the critical case $p=2^{*}$ with $d \geq 3$, we have to use the GagliardoNirenberg inequality in hyperbolic space to establish the following Morawetz estimate:

$$
\iint_{\mathbb{R}^{d+1}} \frac{|u|^{2^{*}}}{t+|x|} d x d t \leq C(E), \quad d \geq 3
$$

Scattering theory and its induction. In order to simply the statement, we introduce the notation

$$
\vec{u}=\left(u,(I-\Delta)^{-\frac{1}{2}} \dot{u}\right)
$$

Then $\vec{u}(t) \in \dot{H}^{1}\left(\mathbb{R}^{d}\right)$ has the following conservation

$$
E(u, t) \triangleq \int_{\mathbb{R}^{d}}\left(|\nabla \vec{u}(t)|^{2}+|\vec{u}(t)|^{2}+F(u(t))\right) d x=E(u, 0) .
$$

Obviously, the above energy is consistent with the original form

$$
\begin{aligned}
E(u, t)= & \int_{\mathbb{R}^{d}}\left(|\nabla u|^{2}+\left|\nabla \sqrt{1-\Delta}^{-1} \dot{u}\right|^{2}+|u|^{2}\right. \\
& \left.+\left|\sqrt{1-\Delta}^{-1} \dot{u}\right|^{2}+F(u)\right) d x \\
= & \int_{\mathbb{R}^{d}}\left(|\nabla u(t)|^{2}+|\dot{u}(t)|^{2}+|u(t)|^{2}+F(u(t))\right) d x .
\end{aligned}
$$


Theorem 5.2 (Brenner-Ginibre-Velo-Nakanishi). Let $d \geq 1, f: \mathbb{C} \rightarrow \mathbb{C}$ satisfy the structure, growth and repulsive conditions. Then (NLKG) has the unique global energy solution $u \in C\left(\mathbb{R} ; H^{1}\left(\mathbb{R}^{d}\right)\right) \cap C^{1}\left(\mathbb{R} ; L^{2}\left(\mathbb{R}^{d}\right)\right)$ with

$$
\begin{cases}\|u\|_{L^{\rho}\left(\mathbb{R} ; B_{\rho, 2}^{\frac{1}{2}}\left(\mathbb{R}^{d}\right)\right)} \leq C\left(E_{0}\right), & d \leq 2, \\ \|u\|_{L^{\rho}\left(\mathbb{R} ; B_{\rho, 2}^{\frac{1}{2}}\left(\mathbb{R}^{d}\right)\right) \cap L \varsigma\left(\mathbb{R} ; B_{\zeta, 2}^{\frac{1}{2}}\left(\mathbb{R}^{d}\right)\right)} \leq C\left(E_{0}\right), & d \geq 3,\end{cases}
$$

where

$$
\rho=2+\frac{4}{d}, \quad \zeta=2+\frac{4}{d-1}
$$

and $E_{0}=E(u, 0)$ is the energy of the solution $u$ at $t=0$.

Moreover, by (5.8), the solution u scatters, i.e., there exists a unique solution $v_{ \pm}$of the following free Klein-Gordon equation

$$
\square v+v=0
$$

such that

$$
\left\|\vec{u}(t)-\vec{v}_{ \pm}(t)\right\|_{H^{1}\left(\mathbb{R}^{d}\right) \times L^{2}\left(\mathbb{R}^{d}\right)} \rightarrow 0, \quad \text { as } t \rightarrow \pm \infty
$$

and the corresponding wave operator

$$
\Omega_{ \pm}: \vec{v}^{ \pm}(0) \triangleq v^{ \pm}(x) \rightarrow \vec{u}(0), \quad H^{1}\left(\mathbb{R}^{d}\right) \times L^{2}\left(\mathbb{R}^{d}\right) \rightarrow H^{1}\left(\mathbb{R}^{d}\right) \times L^{2}\left(\mathbb{R}^{d}\right)
$$

is a homeomorphism from $H^{1}\left(\mathbb{R}^{d}\right) \times L^{2}\left(\mathbb{R}^{d}\right)$ to itself.

For the defocusing energy-critical NLKG, refer to Miao's Book [108.

5.1. Focusing case. First, we consider the Energy-subcritical case:

\section{Motivation:}

$$
\left\{\begin{array}{l}
u_{t t}-\Delta u+u-|u|^{p-1} u=0 \\
\left(u, \partial_{t} u\right)(0)=\left(u_{0}, u_{1}\right) \in H^{1}\left(\mathbb{R}^{d}\right) \times L^{2}\left(\mathbb{R}^{d}\right) .
\end{array}\right.
$$

(1) It is obvious that $u(x, t)=Q(x)$ is the solution of (5.9) with $\left(u, \partial_{t} u\right)(0)=$ $(Q(x), 0)$, where $Q$ is the ground state of elliptic equation

$$
-\Delta Q+Q=|Q|^{p-1} Q, \quad p<1+\frac{4}{d-2} .
$$

From the viewpoint of "phase transition", we conjecture that $u(t, x)=Q$ belongs to the boundary of scattering domain.

(2) Payne and Sattinger proved the global existence/finite time blowup dichotomy for solutions of energies of (NLKG) on bounded smooth domain in $\mathbb{R}^{d}$ below the ground-state energy, by the sign of the functional

$$
K_{1,0}(u) \triangleq \int\left(|\nabla u|^{2}+|u|^{2}-|u|^{p+1}\right) d x .
$$

It is easy to observe that their argument applies to the whole space $\mathbb{R}^{d}$ as soon as one has the local well-posedness for (NLKG) in the energy space.

(3) A simple computation shows

$$
\frac{d}{d t} \int_{\mathbb{R}^{d}}(x \cdot \nabla+\nabla \cdot x) u u_{t} d x=-\left(\int_{\mathbb{R}^{d}}\left(2|\nabla u|^{2}-\frac{d(p-1)}{p+1}|u|^{p+1}\right) d x\right)=-K_{d,-2}(u),
$$

which is useful for the scattering theory.

\section{Variational setting:}


To state the main results, we need to introduce some notation and assumptions for the variational setting.

Let the static energy be

$$
J(\phi)=\frac{1}{2} \int_{\mathbb{R}^{d}}\left(|\nabla \phi|^{2}+|\phi|^{2}\right) d x-\frac{1}{p+1} \int_{\mathbb{R}^{d}}|\phi|^{p+1} d x .
$$

The scaling derivative of the static energy is denoted by

$$
\begin{aligned}
& K_{\alpha, \beta}(\phi)=\left.\frac{d}{d \lambda}\right|_{\lambda=0} J\left(e^{\lambda \alpha} \phi\left(e^{-\beta \lambda} x\right)\right) \\
= & \int_{\mathbb{R}^{d}}\left(\frac{2 \alpha+(d-2) \beta}{2}|\nabla \phi|^{2}+\frac{2 \alpha+d \beta}{2}|\phi|^{2}-\frac{(p+1) \alpha+p d}{p+1}|\phi|^{p+1}\right) d x \\
= & \int_{\mathbb{R}^{d}}(\alpha \varphi-\beta x \cdot \nabla \varphi)\left(-\Delta \varphi+\varphi-|\varphi|^{p-1} \varphi\right) d x .
\end{aligned}
$$

Let

$$
\Omega \triangleq\left\{(\alpha, \beta) \mid \alpha \geq 0,(\alpha, \beta) \neq(0,0), \begin{array}{c}
2 \alpha+(d-2) \beta \geq 0 \\
2 \alpha+d \beta \geq 0,
\end{array}\right\}
$$

we consider the constrained minimization problem

$$
m_{\alpha, \beta}=\inf \left\{J(\varphi) \mid \varphi \in H^{1}\left(\mathbb{R}^{d}\right) \backslash\{0\}, K_{\alpha, \beta}=0\right\} .
$$

Proposition 5.3 (Parameter independent). If $(\alpha, \beta) \in \Omega$, then $m_{\alpha, \beta}$ is independent of $(\alpha, \beta)$ and it is attained

$$
m_{\alpha, \beta}=J(Q),
$$

where $Q$ is the ground state of the elliptic equation

$$
-\Delta Q+Q=|Q|^{p-1} Q .
$$

The solutions start from the following subsets of the energy space:

$$
K_{\alpha, \beta}^{ \pm}=\left\{\left(u_{0}, u_{1}\right) \mid E\left(u_{0}, u_{1}\right)<E(Q, 0)=J(Q), K_{\alpha, \beta}(u)_{<} \geq_{0}\right\} .
$$

Proposition 5.4 (Parameter independence of the splitting). For $(\alpha, \beta) \in \Omega, K_{\alpha, \beta}^{ \pm}$ is independent of $(\alpha, \beta)$.

Lemma 5.5 (Uniform bounds on $K)$. Assume that $(\alpha, \beta) \in \Omega$ and $(d, \alpha) \neq(2,0)$, then $\exists \delta=\delta(\alpha, \beta, d, p)>0$, s.t. $\forall \varphi \in H^{1}, J(\varphi)<m$, we have

$$
K_{\alpha, \beta} \geq \min \left\{\bar{\mu}(m-J(\varphi)), \delta K_{\alpha, \beta}^{Q}(\varphi)\right\}, \text { or } K_{\alpha, \beta} \leq-\bar{\mu}(m-J(\varphi)),
$$

where $\bar{\mu}=\max \{2 \alpha+(d-2) \beta, 2 \alpha+d \beta\}$ and the quadratic part

$$
K_{\alpha, \beta}^{Q}(\varphi)=\frac{2 \alpha+(d-2) \beta}{2}\|\nabla \varphi\|_{2}^{2}+\frac{2 \alpha+d \beta}{2}\|\varphi\|_{2}^{2} .
$$

Theorem 5.6 (Focusing energy-subcritical, Ibrahim, Masmoudi and Nakanishi 64]). For $(\alpha, \beta) \in \Omega$.

(1) If $(u(0), \dot{u}(0)) \in K_{\alpha, \beta}^{-}$, then the solution $u$ extends neither for $t \rightarrow \infty$ nor for $t \rightarrow-\infty$ as the unique strong solution in $H^{1} \times L^{2}$. 
(2) If $(u(0), \dot{u}(0)) \in K_{\alpha, \beta}^{+}$, then the solution $u$ scatters both in $t \rightarrow \pm \infty$ in the energy space. In other words, $u$ is a global solution and there are $v_{ \pm}$ satisfying

$$
\begin{gathered}
v_{t t}-\Delta v+v=0, \\
\left\|(u, \dot{u})-\left(v_{ \pm}, \dot{v}_{ \pm}\right)\right\|_{H^{1} \times L^{2}} \rightarrow 0, \text { as } t \rightarrow \pm \infty .
\end{gathered}
$$

\section{Energy-critical NLKG}

Let $d \geq 3$, we consider

$$
\left\{\begin{array}{l}
u_{t t}-\Delta u+u=|u|^{\frac{4}{d-2}} u \\
u(0)=u_{0}(x) \in H^{1} \\
u_{t}(0)=u_{1}(x) \in L^{2}
\end{array}\right.
$$

where $u: \mathbb{R}^{1+d} \mapsto \mathbb{R}$.

It is obvious that $u(t, x)=e^{i t} Q$ is the solution of energy-critical NLKG, where $Q$ is the ground state of elliptic equation

$$
-\Delta Q=|Q|^{\frac{4}{d-2}} Q .
$$

From the viewpoint of phase transition, we conjecture that $u(t, x)=e^{i t} Q$ corresponds to the boundary of scattering domain.

Let the static energy be

$$
J(\phi)=\frac{1}{2} \int_{\mathbb{R}^{d}}\left(|\nabla \phi|^{2}+|\phi|^{2}\right) d x-\frac{1}{p+1} \int_{\mathbb{R}^{d}}|\phi|^{p+1} d x .
$$

In the critical case, we also need the modified static energy

$$
J^{(0)}(\phi):=\frac{1}{2} \int_{\mathbb{R}^{d}}|\nabla \phi|^{2} d x-\frac{1}{p+1} \int_{\mathbb{R}^{d}}|\phi|^{p+1} d x .
$$

The scaling derivative of the static energy is denoted by

$$
\begin{aligned}
& K_{\alpha, \beta}(\phi)=\left.\frac{d}{d \lambda}\right|_{\lambda=0} J\left(e^{\lambda \alpha} \phi\left(e^{-\beta \lambda} x\right)\right) \\
= & \int_{\mathbb{R}^{d}}\left(\frac{2 \alpha+(d-2) \beta}{2}|\nabla \phi|^{2}+\frac{2 \alpha+d \beta}{2}|\phi|^{2}-\frac{(p+1) \alpha+p d}{p+1}|\phi|^{p+1}\right) d x \\
= & \int_{\mathbb{R}^{d}}(\alpha \varphi-\beta x \cdot \nabla \varphi)\left(-\Delta \varphi+\varphi-|\varphi|^{p-1} \varphi\right) d x .
\end{aligned}
$$

\section{Variational Characterization of Ground State $Q$}

The ground state $Q$ has the following characterization

$$
\begin{aligned}
m_{\alpha, \beta} & \triangleq \inf \left\{J(\phi) \mid \phi \in H^{1} \backslash\{0\}, K_{\alpha, \beta}(\phi)=0\right\} \\
& =\inf \left\{\left.J^{(0)}(\phi)\left|\phi \in \dot{H}^{1} \backslash\{0\},-\Delta \phi=\right| \phi\right|^{\frac{4}{d-2}} \phi\right\} \\
& =J^{(0)}(Q) .
\end{aligned}
$$

where $(\alpha, \beta) \in \Omega=\left\{(\alpha, \beta) \mid \alpha \geq 0,(\alpha, \beta) \neq(0,0), \begin{array}{c}2 \alpha+(d-2) \beta \geq 0, \\ 2 \alpha+d \beta \geq 0,\end{array}\right\}$ 
The solutions start from the following subsets of the energy space:

$$
K_{\alpha, \beta}^{ \pm}=\left\{\left(u_{0}, u_{1}\right) \mid E\left(u_{0}, u_{1}\right)<J^{(0)}(Q), \quad K_{\alpha, \beta}(u)_{<}^{\geq}\right\} .
$$

Proposition 5.7 (Parameter independence of the splitting). For $(\alpha, \beta) \in \Lambda, K_{\alpha, \beta}^{ \pm}$ is independent of $(\alpha, \beta)$.

Lemma 5.8 (Uniform bounds on $K)$. Assume that $(\alpha, \beta) \in \Omega$, then $\exists \delta=\delta(\alpha, \beta, d, p)>$ 0 , s.t. $\forall \varphi \in H^{1}, J(\varphi)<m$, we have

$$
K_{\alpha, \beta} \geq \min \left\{\bar{\mu}(m-J(\varphi)), \delta K_{\alpha, \beta}^{Q}(\varphi)\right\}, \text { or } K_{\alpha, \beta} \leq-\bar{\mu}(m-J(\varphi)),
$$

where $\bar{\mu}=\max \{2 \alpha+(d-2) \beta, 2 \alpha+d \beta\}$ and the quadratic part

$$
K_{\alpha, \beta}^{Q}(\varphi)=\frac{2 \alpha+(d-2) \beta}{2}\|\nabla \varphi\|_{2}^{2}+\frac{2 \alpha+d \beta}{2}\|\varphi\|_{2}^{2} .
$$

Theorem 5.9 (Focusing energy-critical NLKG, Ibrahim, Masmoudi and Nakanishi 64]). For $(\alpha, \beta) \in \Omega$.

(1) If $(u(0), \dot{u}(0)) \in K_{\alpha, \beta}^{-}$, then the solution $u$ extends neither for $t \rightarrow \infty$ nor for $t \rightarrow-\infty$ as the unique strong solution in $H^{1} \times L^{2}$.

(2) If $(u(0), \dot{u}(0)) \in K_{\alpha, \beta}^{+}$, then the solution $u$ scatters both in $t \rightarrow \pm \infty$ in the energy space. In other words, $u$ is a global solution and there are $v_{ \pm}$ satisfying

$$
\begin{gathered}
v_{t t}-\Delta v+v=0, \\
\left\|(u, \dot{u})-\left(v_{ \pm}, \dot{v}_{ \pm}\right)\right\|_{H^{1} \times L^{2}} \rightarrow 0, \text { as } t \rightarrow \pm \infty .
\end{gathered}
$$

Remark 5.10. One can refer this part to the book by Nakanishi and Schlag [113] or see Miao's lecture on nonlinear Klein-Gordon equation.

\section{Proof of Defocusing EnERgy-CRItical NLW}

Theorem 6.1. Let $d \geq 3$. Given $\left(u_{0}, u_{1}\right) \in \dot{H}^{1}\left(\mathbb{R}^{d}\right) \times L^{2}\left(\mathbb{R}^{d}\right)$. Then, there is a unique global strong solution $u$ to

$$
(\mathrm{NLW}) \quad\left\{\begin{array}{l}
\partial_{t t} u-\Delta u+|u|^{\frac{4}{d-2}} u=0, \\
\left(u, \partial_{t} u\right)(0)=\left(u_{0}, u_{1}\right)
\end{array}\right.
$$

Moreover, the solution $u$ obeys the estimate

$$
\int_{\mathbb{R}} \int_{\mathbb{R}^{d}}|u(t, x)|^{\frac{2(d+1)}{d-2}} d x d t \leq C\left(\left\|\left(u_{0}, u_{1}\right)\right\|_{\dot{H}_{x}^{1} \times L_{x}^{2}}\right) .
$$

And so the solution scatters.

Notation: Let $z=(x, t)$ denote the space-time coordinate

(1) Cone $K\left(z_{0}\right)$ and cut-off of Cone $K_{S}^{T}$ :

$$
\begin{aligned}
K\left(z_{0}\right) & =\left\{z=(x, t)|| x-x_{0} \mid \leq t_{0}-t, 0 \leq t \leq t_{0}\right\} \\
K_{S}^{T} & =\left\{z=(x, t) \mid(x, t) \in K\left(z_{0}\right), S \leq t \leq T\right\}
\end{aligned}
$$

(2) Lateral Surface $M_{S}^{T}\left(z_{0}\right)$ and $\partial K_{S}^{T}$ :

$$
\begin{aligned}
M_{S}^{T}\left(z_{0}\right) & =\left\{z=(x, t)|| x-x_{0} \mid=t_{0}-t, S \leq t \leq T\right\} \\
\partial K_{S}^{T} & =D_{S}\left(z_{0}\right) \cup D_{T}\left(z_{0}\right) \cup M_{S}^{T}
\end{aligned}
$$


(3) Section: $D_{t}\left(z_{0}\right)=\left\{z=(x, t) \mid(x, t) \in K\left(z_{0}\right), t\right.$ fixed $\}$

(4) ST Slab: $Q_{S}^{T}=\{z=(x, t) \mid(x, t) \in Q, S \leq t \leq T\}$ for any $Q \subset \mathbb{R}^{d} \times \mathbb{R}$ and $S \leq T$

For any given function $u$ on $K\left(z_{0}\right)$, we introduce $\left(2^{*}=\frac{2 d}{d-2}\right)$

(1) Energy-Momentum density

$$
e(u)=\left(\frac{1}{2}\left|u_{t}\right|^{2}+\frac{1}{2}|\nabla u|^{2}+\frac{1}{2^{*}}|u|^{2^{*}},-\left(u_{t} \nabla u\right)\right)
$$

(2) Local energy

$$
E\left(u, D_{T}\left(z_{0}\right)\right)=\int_{D_{T}\left(z_{0}\right)}\left(\frac{1}{2}\left|u_{t}\right|^{2}+\frac{1}{2}|\nabla u|^{2}+\frac{1}{2^{*}}|u|^{2^{*}}\right) d x
$$

(3) Energy flux density

$$
d z_{0}(u)=\frac{1}{2}\left|\frac{y}{|y|} u_{t}-\nabla u\right|^{2}+\frac{1}{2^{*}}|u|^{2^{*}}, \quad y=x-x_{0}
$$

(4) Emission energy from side $M_{S}^{T}$

$$
\operatorname{Flux}\left(u, M_{S}^{T}\left(z_{0}\right)\right)=\int_{M_{S}^{T}\left(z_{0}\right)} d z_{0}(u) d \sigma
$$

\section{Energy conservative and its local form-Basic Fact I:}

Multiplying (NLW) with $\partial_{t} u$ (generation element of time translation invariance),

$$
\operatorname{div}_{t, x} e(u)=\left(\frac{1}{2}\left|u_{t}\right|^{2}+\frac{1}{2}|\nabla u|^{2}+\frac{1}{2^{*}}|u|^{2^{*}}\right)_{t}-\operatorname{div}\left(\nabla u u_{t}\right)=0,
$$

then integrating on $K\left(z_{0}\right)$ and $\mathbb{R}^{d}$, we can obtain

$$
\begin{gathered}
\left.E\left(u, D_{T}\left(z_{0}\right)\right)+\frac{1}{\sqrt{2}} \operatorname{Flux}\left(u, M_{S}^{T}\left(z_{0}\right)\right)=E\left(u, D_{S}\left(z_{0}\right)\right)\right), \\
E\left(u, u_{t}\right)=\int_{\mathbb{R}^{d}}\left(\frac{1}{2}\left|u_{t}\right|^{2}+\frac{1}{2}|\nabla u|^{2}+\frac{1}{2^{*}}|u|^{2^{*}}\right) d x=E\left(u_{0}, u_{1}\right) .
\end{gathered}
$$

\section{The local form of conformal identity- Basic Fact II:}

Multiplying 6.1 with $\left(t \partial_{t}+x \cdot \nabla+\frac{d-1}{2}\right) u$, which is the generation element of the scaling transformation $u_{\varepsilon}(t, x) \rightarrow \varepsilon^{\frac{d-1}{2}} u(\varepsilon t, \varepsilon x)$, we can obtain

$$
\begin{aligned}
& \operatorname{div}_{t, x}\left(t Q_{0}+\frac{d-1}{2} u_{t} u,-t P_{0}\right)+R_{0}=0 \\
& \text { or } \\
& \partial_{t}\left(t Q_{0}+\frac{d-1}{2} u_{t} u\right)-\operatorname{div}\left(t P_{0}\right)+R_{0}=0
\end{aligned}
$$


where

$$
\begin{aligned}
Q_{0} & =\frac{1}{2}\left|u_{t}\right|^{2}+\frac{1}{2}|\nabla u|^{2}+\frac{1}{2^{*}}|u|^{2^{*}}+u_{t}\left(\frac{x}{t} \cdot \nabla u\right), \quad R_{0}=\frac{|u|^{2^{*}}}{d} \\
P_{0} & =\frac{x}{t}\left(\frac{\left|u_{t}\right|^{2}-|\nabla u|^{2}}{2}-\frac{|u|^{2^{*}}}{2^{*}}\right)+\nabla u\left(u_{t}+\frac{x}{t} \cdot \nabla u+\frac{d-1}{2} \frac{u}{t}\right) .
\end{aligned}
$$

Non concentration of potential energy-Basic Fact III:

If $u(t, x)$ is the regularized solution on $K\left(z_{0}\right) \backslash\left\{z_{0}\right\}\left(u \in C^{2}\left(\left(0, t_{0}\right) \times \mathbb{R}^{d}\right)\right)$, then we have

$$
\lim _{s \rightarrow t_{0}} \int_{D_{S}\left(z_{0}\right)}|u|^{2^{*}} d x=0 .
$$

The sketch of proof on existence of global smooth solution:

Step 1: By the finite propagation speed, we can induce the above problem to the problem with compact supported data $\left(u_{0}(x), u_{1}(x)\right)$.

Step 2: Nonlinear estimate

$$
\left\||u|^{2^{*}-2} u\right\|_{L^{q^{\prime}}\left([s, \tau] ; B_{q^{\prime}}^{1 / 2}\left(D_{t}\left(z_{0}\right)\right)\right)} \leq \sup _{t \in[s, \tau]}\|u\|_{L^{2^{*}}\left(D_{t}\left(z_{0}\right)\right)}^{\frac{2^{*}-2}{d-1}}\|u\|_{L^{q}\left([s, \tau] ; B_{q}^{1 / 2}\left(D_{t}\left(z_{0}\right)\right)\right)}^{1+\frac{d-2}{d-1}\left(2^{*}-2\right)} .
$$

This estimate together with the Strichartz estimates implies that

$$
\|u\|_{L^{q}\left([s, \tau] ; \dot{B}_{q}^{1 / 2}\left(D_{t}\left(z_{0}\right)\right)\right)} \leq C\left(E_{0}\right)+\varepsilon\|u\|_{L^{q}\left([s, \tau] ; \dot{B}_{q}^{1 / 2}\left(D_{t}\left(z_{0}\right)\right)\right)}^{\gamma},
$$

where

$$
q=\frac{2(d+1)}{d-1}, \quad \gamma>1
$$

Step 3: Continuous argument (Compactness $\Longrightarrow$ GWP)

By the compactness and the finite propagation speed, it suffices to show that $u(t, x)$ is smooth on the neighborhood region of $\left(t_{0}, x_{0}\right)$.

\section{The proof of scattering result}

Step 1: Induction. Given $\left(u, u_{t}\right) \in C\left(\mathbb{R} ; \dot{H}^{1}\right) \times C\left(\mathbb{R} ; L^{2}\left(\mathbb{R}^{d}\right)\right)$, it suffices to show that

$$
\left(u, u_{t}\right) \in L^{q}\left(\mathbb{R} ; \dot{B}_{q}^{\frac{1}{2}}\left(\mathbb{R}^{d}\right)\right) \times L^{q}\left(\mathbb{R} ; \dot{B}_{q}^{-\frac{1}{2}}\left(\mathbb{R}^{d}\right)\right) .
$$

By LWP thery, we know that

$$
\left(u, u_{t}\right) \in L_{l o c}^{q}\left(\mathbb{R} ; \dot{B}_{q}^{\frac{1}{2}}\left(\mathbb{R}^{d}\right)\right) \times L_{l o c}^{q}\left(\mathbb{R} ; \dot{B}_{q}^{-\frac{1}{2}}\left(\mathbb{R}^{d}\right)\right),
$$

it suffices to show for sufficiently large $T_{0}>0$

$$
\left(u, u_{t}\right) \in L^{q}\left(\left[T_{0}, \infty\right) ; \dot{B}_{q}^{\frac{1}{2}}\left(\mathbb{R}^{d}\right)\right) \times L^{q}\left(\left[T_{0}, \infty\right) ; \dot{B}_{q}^{-\frac{1}{2}}\left(\mathbb{R}^{d}\right)\right) .
$$

Step 2: We can further reduce 6.5 in essence to prove

$$
\lim _{t \rightarrow+\infty} g(t) \triangleq \frac{1}{2^{*}} \int_{\mathbb{R}^{d}}|u|^{2^{*}} d x=0
$$


In fact, by Strichartz estimate and nonlinear estimates, we have

$$
\begin{aligned}
\|u\|_{L^{q}\left(\left[T_{0}, T\right] ; \dot{B}_{q}^{\frac{1}{2}}\right)} & \lesssim E\left(u_{0}, u_{1}\right)^{\frac{1}{2}}+\sup _{T_{0} \leq t \leq T}\|u\|_{2^{*}}^{(1-\alpha)\left(2^{*}-2\right)}\|u\|_{L^{q}\left(\left[T_{0}, T\right] ; \dot{B}_{q}^{\frac{1}{2}}\right)}^{1+\alpha\left(2^{*}-2\right)} \\
& \lesssim E\left(u_{0}, u_{1}\right)^{\frac{1}{2}}+\varepsilon_{0}^{(1-\alpha)\left(2^{*}-2\right)}\|u\|_{L^{q}\left(\left[T_{0}, T\right] ; \dot{B}_{q}^{\frac{1}{2}}\right)}^{1+\alpha\left(2^{*}-2\right)},
\end{aligned}
$$

where $\alpha=\frac{d-2}{d-1}$. By the continuous argument, we can show that

$$
\|u\|_{L^{q}\left(\left[T_{0}, T\right] ; \dot{B}_{q}^{\frac{1}{2}}\left(\mathbb{R}^{d}\right)\right)} \leq 2 C E\left(u_{0}, u_{1}\right)^{\frac{1}{2}} \Rightarrow\|u\|_{L^{q}\left(\left[T_{0}, \infty\right) ; \dot{B}_{q}^{\frac{1}{2}}\left(\mathbb{R}^{d}\right)\right)}<\infty
$$

Step 3: Proof of 6.6) ( FPS and conformal scaling transform.).

First of all, for sufficiently large $R>R_{0}$, we have

$$
\int_{|x| \geq R} e\left(u_{0}, u_{1}\right) d x \leq \frac{\varepsilon_{0}}{8}, \quad e\left(u, u_{t}\right)=\frac{1}{2}|\nabla u|^{2}+\frac{1}{2}\left|u_{t}\right|^{2}+\frac{1}{2^{*}}|u|^{2^{*}} .
$$

Now we integrate the following identity

$$
\partial_{t}\left(e\left(u, u_{t}\right)\right)-\nabla \cdot\left(\nabla u u_{t}\right)=0,
$$

over the outside of the forward light cone, and obtain

$$
\int_{|x| \geq R+t} e\left(u, u_{t}\right) d x+\frac{1}{\sqrt{2}} \operatorname{Flux}\left(u, M_{0}^{t}\right)=\int_{|x| \geq R} e\left(u_{0}, u_{1}\right) d x \leq \frac{\varepsilon_{0}}{8},
$$

where

$$
\operatorname{Flux}\left(u, M_{0}^{t}\right)=\int_{M_{0}^{t}}\left(\frac{1}{2}\left|\frac{x}{|x|} u_{t}+\nabla u\right|^{2}+\frac{1}{2^{*}}|u|^{2^{*}}\right) d \sigma \geq 0,
$$

which implies that

$$
\frac{1}{2^{*}} \int_{|x| \geq R+t}|u(t, x)|^{2^{*}} d x \leq \frac{\varepsilon_{0}}{4} .
$$

Now it suffices to show that for any $t \geq T_{0}$

$$
\frac{1}{2^{*}} \int_{|x| \leq R+t}|u(t, x)|^{2^{*}} d x \leq \frac{\varepsilon_{0}}{4} .
$$

By the time translation invariance $(t \rightarrow t+R)$, we only need to show that for any $t \geq T_{0}$

$$
\frac{1}{2^{*}} \int_{|x| \leq t}|u(t, x)|^{2^{*}} d x \leq \frac{\varepsilon_{0}}{2}
$$

Integrating the local form of conformal identity 6.4 over the cone $M_{\varepsilon T}^{T}$, we can obtain 6.7).

\section{Proof of Sacttering/Blowup dichotomy (Theorem 2.5)}

For this section, we refer to Kenig's lecture notes [75, 76] and Kenig-Merle [77. To describe the proof of Theorem 2.5, we start out by a brief review of the local Cauchy problem. To do this, we introduce some definitions and background materials. 
Definition 7.1 (Strong solution). Let $I$ be a nonempty time interval including $t_{0}$. A function $u: I \times \mathbb{R}^{d} \rightarrow \mathbb{R}$ is a strong solution to problem $(2.9)$, if

$$
\left(u, u_{t}\right) \in C_{t}^{0}\left(J ; \dot{H}^{1}\left(\mathbb{R}^{d}\right) \times \dot{L}^{2}\left(\mathbb{R}^{d}\right), \text { and } u \in L_{t, x}^{\frac{2(d+1)}{d-2}}\left(J \times \mathbb{R}^{d}\right)\right.
$$

for any compact $J \subset I$ and for each $t \in I$ such that

$$
\left(\begin{array}{l}
u(t) \\
\dot{u}(t)
\end{array}\right)=V_{0}\left(t-t_{0}\right)\left(\begin{array}{l}
u_{0}(x) \\
u_{1}(x)
\end{array}\right)-\int_{t_{0}}^{t} V_{0}(t-s)\left(\begin{array}{c}
0 \\
f(u(s))
\end{array}\right) \mathrm{d} s,
$$

where

$$
V_{0}(t)=\left(\begin{array}{ll}
\dot{K}(t) & K(t) \\
\ddot{K}(t) & \dot{K}(t)
\end{array}\right), \quad K(t)=\frac{\sin (t \omega)}{\omega}, \quad \omega=(-\Delta)^{1 / 2},
$$

and the dot denotes the time derivative. We refer to the interval $I$ as the lifespan of $u$. We say that $u$ is a maximal-lifespan solution if the solution cannot be extended to any strictly larger interval. We say that $u$ is a global solution if $I=\mathbb{R}$.

The solution lies in the space $L_{t, x}^{\frac{2(d+1)}{d-2}}\left(I \times \mathbb{R}^{d}\right)$ locally in time is a natural fact since by Strichartz estimate, the linear flow always lies in this space. We define the scattering size of a solution $u$ to problem 2.9 ) on a time interval $I$ by

$$
\|u\|_{S(I)}:=\|u\|_{L_{t, x}^{\frac{2(d+1)}{d-2}}\left(I \times \mathbb{R}^{d}\right)} .
$$

Standard arguments show that if a solution $u$ of problem 2.9 is global, with $S_{\mathbb{R}}(u)<+\infty$, then it scatters, i.e. there exist unique $\left(v_{0}^{ \pm}, v_{1}^{ \pm}\right) \in \dot{H}^{1}\left(\mathbb{R}^{d}\right) \times L^{2}\left(\mathbb{R}^{d}\right)$ such that

$$
\left\|\left(\begin{array}{c}
u(t) \\
\dot{u}(t)
\end{array}\right)-V_{0}(t)\left(\begin{array}{c}
v_{0}^{ \pm} \\
v_{1}^{ \pm}
\end{array}\right)\right\|_{\dot{H}^{1}\left(\mathbb{R}^{d}\right) \times L^{2}\left(\mathbb{R}^{d}\right)} \longrightarrow 0, \quad \text { as } \quad t \longrightarrow \pm \infty .
$$

The notion closely associated with scattering is the definition of blowup:

Definition 7.2 (Blowup). We call that a maximal-lifespan solution $u: I \times \mathbb{R}^{d} \rightarrow \mathbb{R}$ of problem (2.9) blows up forward in time if there exists a time $\tilde{t}_{0} \in I$ such that $\|u\|_{\left.S\left(\tilde{t}_{0}, \sup I\right)\right)}=+\infty$. Similarly, $u(t, x)$ blows up backward in time if there exists a time $\tilde{t}_{0} \in I$ such that $\|u\|_{S\left(\inf I, \tilde{t}_{0}\right]}=+\infty$.

7.1. Local Cauchy problem. We first consider the associated linear problem,

$$
\left\{\begin{array}{l}
w_{t t}-\Delta w=h, \\
\left(w(0), \partial_{t} w(0)\right)=\left(w_{0}, w_{1}\right) \in \dot{H}^{1}\left(\mathbb{R}^{d}\right) \times L^{2}\left(\mathbb{R}^{d}\right) .
\end{array}\right.
$$

As is well known, the solution is given by

$$
\begin{aligned}
w(x, t) & =\cos (t \sqrt{-\Delta}) w_{0}+\frac{\sin (t \sqrt{-\Delta})}{\sqrt{-\Delta}} w_{1}+\int_{0}^{t} \frac{\sin ((t-s) \sqrt{-\Delta})}{\sqrt{-\Delta}} h(s) d s \\
& \triangleq S(t)\left(w_{0}, w_{1}\right)+\int_{0}^{t} \frac{\sin ((t-s) \sqrt{-\Delta})}{\sqrt{-\Delta}} h(s) d s .
\end{aligned}
$$

The normal Strichartz norms

$$
\|f\|_{S(I)}:=\|f\|_{L_{t, x}^{\frac{2(d+1)}{d-2}}\left(I \times \mathbb{R}^{d}\right)},\|f\|_{W(I)}:=\|f\|_{L_{t, x}^{\frac{2(d+1)}{d-1}}\left(I \times \mathbb{R}^{d}\right)} .
$$


Theorem 7.3 (Strichartz estimates, [57]). We have

$$
\begin{aligned}
& \quad \sup _{t}\left\|\left(w(t), \partial_{t} w(t)\right)\right\|_{\dot{H}^{1} \times L^{2}}+\left\|D^{\frac{1}{2}} w\right\|_{W(\mathbb{R})}+\left\|\partial_{t} D^{-\frac{1}{2}} w\right\|_{W(\mathbb{R})} \\
& \quad+\|w\|_{S(\mathbb{R})}+\|w\|_{L_{t}^{\frac{d+2}{d-2}} L_{x}^{\frac{2(d+2)}{d-2}}} \\
& \leq C\left\|\left(w_{0}, w_{1}\right)\right\|_{\dot{H}^{1} \times L^{2}}+C\left\|D^{\frac{1}{2}} h\right\|_{L_{t, x}^{\frac{2(d+1)}{d+3}}}
\end{aligned}
$$

Because of the appearance of $D^{\frac{1}{2}}$ in these estimates, we also need to use the following version of the chain rule for fractional derivatives (see [81]).

Lemma 7.4 (Chain rule for fractional derivatives). Assume $F \in C^{2}, F(0)=$ $F^{\prime}(0)=0$, and that for all $a, b$, we have

$$
\left|F^{\prime}(a+b)\right| \leq C\left(\left|F^{\prime}(a)\right|+\left|F^{\prime}(b)\right|\right),\left|F^{\prime \prime}(a+b)\right| \leq C\left(\left|F^{\prime \prime}(a)\right|+\left|F^{\prime \prime}(b)\right|\right) .
$$

Then, for

we have

$$
\alpha \in(0,1), \frac{1}{p}=\frac{1}{p_{1}}+\frac{1}{p_{2}}=\frac{1}{r_{1}}+\frac{1}{r_{2}}+\frac{1}{r_{3}}
$$

$$
\begin{gathered}
\left\|D^{\alpha} F(u)\right\|_{L^{p}} \leq C\left\|F^{\prime}(u)\right\|_{L^{p_{1}}}\left\|D^{\alpha} u\right\|_{L^{p_{2}}}, \\
\left\|D^{\alpha}(F(u)-F(v))\right\|_{L^{p}} \leq C\left(\left\|F^{\prime}(u)\right\|_{L^{p_{1}}}+\left\|F^{\prime}(v)\right\|_{L^{p_{1}}}\right)\left\|D^{\alpha}(u-v)\right\|_{L^{p_{2}}} \\
+C\left(\left\|F^{\prime \prime}(u)\right\|_{L^{r_{1}}}+\left\|F^{\prime \prime}(v)\right\|_{L^{r_{1}}}\right) \cdot\left(\left\|D^{\alpha} u\right\|_{L^{r_{2}}}+\left\|D^{\alpha} v\right\|_{L^{r_{2}}}\right)\|u-v\|_{L^{r_{3}}} .
\end{gathered}
$$

Local theory Using these estimates, we obtain

Theorem 7.5 (Local theory, [57, 72, 77]). Assume

$$
\left(u_{0}, u_{1}\right) \in \dot{H}^{1} \times L^{2},\left\|\left(u_{0}, u_{1}\right)\right\|_{\dot{H}^{1} \times L^{2}} \leq A .
$$

Then, there exists $\delta=\delta(A)$ such that if

$$
\left\|S(t)\left(u_{0}, u_{1}\right)\right\|_{S(I)} \leq \delta,
$$

there exists a unique solution to 2.90 in $I \times \mathbb{R}^{d}$ s.t. $\left(u, \partial_{t}\right) \in C\left(I ; \dot{H}^{1} \times L^{2}\right)$, and

$$
\left\|D^{\frac{1}{2}} u(t)\right\|_{W(I)}+\left\|\partial_{t} D^{-\frac{1}{2}} u\right\|_{W(I)} \leq C(A), \text { and }\|u\|_{S(I)} \leq 2 \delta .
$$

Moreover, $\left(u_{0}, u_{1}\right) \mapsto\left(u, \partial_{t} u\right) \in C\left(I ; \dot{H}^{1} \times L^{2}\right)$ is Lipschitz.

Remark 7.6. (i) (Small data theory) From Strichartz estimate and Sobolev embedding, $\exists \tilde{\delta}$, s.t. if $\left\|\left(u_{0}, u_{1}\right)\right\|_{\dot{H}^{1} \times L^{2}} \leq \tilde{\delta}$, the hypothesis of Theorem 7.5 holds for $I=\mathbb{R}$. Moreover, $u$ scatters in the following sense: there exists $\left(u_{0}^{ \pm}, u_{1}^{ \pm}\right) \in \dot{H}^{1} \times L^{2}$ such that

$$
\lim _{t \rightarrow \pm \infty}\left\|\left(u(t), \partial_{t} u(t)\right)-S(t)\left(u_{0}^{ \pm}, u_{1}^{ \pm}\right)\right\|_{\dot{H}^{1} \times L^{2}}=0 .
$$

(ii) (Maximal life interval) It is easy to see that given $\left(u_{0}, u_{1}\right) \in \dot{H}^{1} \times L^{2}$, there exists time interval $I$ such that $\left\|S(t)\left(u_{0}, u_{1}\right)\right\|_{S(I)} \leq \delta$, so the Theorem 7.5 applies on $I$. Moreover, here exists a maximal interval $I^{*}=\left(-T_{-}\left(u_{0}\right), T_{+}\left(u_{0}\right)\right)$ where

$$
u \in C\left(I^{\prime} ; \dot{H}^{1}\right) \cap\left\{\nabla u \in S\left(I^{\prime}\right)\right\} \text { of all } I^{\prime} \subset \subset I^{*} \text { is defined. }
$$

We call $I^{*}$ the maximal interval of existence. 
(iii) (Blowup criterion) If $T_{+}\left(u_{0}\right)<+\infty$, we must have

$$
\|u\|_{S\left[0, T_{+}\left(u_{0}, u_{1}\right)\right)}=+\infty .
$$

(iv)(Energy conservation) For $t \in I^{*}$, we have

$$
E\left(u_{0}, u_{1}\right) \triangleq \frac{1}{2} \int\left(\left|\nabla u_{0}\right|^{2}+u_{1}^{2}\right) d x-\frac{1}{2^{*}} \int\left|u_{0}\right|^{2^{*}} d x=E\left(u(t), \partial_{t} u(t)\right) .
$$

(v) (Momentum conservation) For $t \in I^{*}$, we have

$$
\int \nabla u(t) \cdot \partial_{t} u(t) d x=\int \nabla u_{0} \cdot u_{1} d x
$$

which is a very important conserved quantity in the energy space, and is crucial to be able to treat non-radial data.

Proof of blowup criterion. If 7.11 is not true, then

$$
M:=\|u\|_{S\left[0, T_{+}\left(u_{0}, u_{1}\right)\right)}<+\infty .
$$

For $\varepsilon>0$ to be chosen, partition $\left[0, T_{+}\left(u_{0}, u_{1}\right)\right)=\bigcup_{j=1}^{\gamma(\varepsilon, M)} I_{j}$, so that

$$
\|u\|_{S\left(I_{j}\right)} \leq \varepsilon
$$

If we write $I_{j}=\left[t_{j}, t_{j+1}\right)$, using Strichartz estimate, we have

$$
\sup _{t \in I_{j}}\left\|\left(u, \partial_{t} u\right)(t)\right\|_{\dot{H}^{1} \times L^{2}}+\left\|D^{\frac{1}{2}} u\right\|_{W\left(I_{j}\right)} \leq C\left\|\left(u_{0}, u_{1}\right)\right\|_{\dot{H}^{1} \times L^{2}}+C\|u\|_{S\left(I_{j}\right)}^{\frac{4}{d-2}}\left\|D^{\frac{1}{2}} u\right\|_{W\left(I_{j}\right)} .
$$

If $C \varepsilon^{\frac{4}{d-2}} \leq \frac{1}{2}$, we can show by continuous argument

$$
\sup _{t \in\left[0, T_{+}\left(u_{0}, u_{1}\right)\right)}\left\|\left(u, \partial_{t} u\right)(t)\right\|_{\dot{H}^{1} \times L^{2}}+\left\|D^{\frac{1}{2}} u\right\|_{W\left(\left[0, T_{+}\left(u_{0}, u_{1}\right)\right)\right)} \leq C(M) .
$$

Choose now $\left\{t_{n}\right\} \nearrow T_{+}\left(u_{0}, u_{1}\right)$ and show again using Strichartz estimate

$$
\left\|S\left(t-t_{n}\right)\left(u\left(t_{n}\right), \partial_{t} u\left(t_{n}\right)\right)\right\|_{S\left(t_{n}, T_{+}\left(u_{0}, u_{1}\right)\right)} \leq \frac{\delta}{2} .
$$

But then by continuous, we have for some $\varepsilon_{0}>0$

$$
\left\|S\left(t-t_{n}\right)\left(u\left(t_{n}\right), \partial_{t} u\left(t_{n}\right)\right)\right\|_{S\left(t_{n}, T_{+}\left(u_{0}, u_{1}\right)+\varepsilon_{0}\right)} \leq \delta,
$$

which, by the Theorem 7.5 contradicts the definition of $T_{+}\left(u_{0}, u_{1}\right)$.

Perturbation Theorem We next turn to another fundamental result in the "local Cauchy theory", the so called "Perturbation Theorem". More precisely, given an approximate equation

$$
\partial_{t t} \tilde{u}-\Delta \tilde{u}=|\tilde{u}|^{\frac{4}{d-2}} \tilde{u}+e
$$

to (FNLW), with $e$ small in sense of suitable time-space norm and $\left(\tilde{u}_{0}, \tilde{u}_{1}\right)$ is close to $\left(u_{0}, u_{1}\right)$ in energy space, is it possible to show that solution $u$ to (FNLW) stays very close to $\tilde{u}$ ? Note that the question of continuous dependence on the data corresponds to the case $e=0$. 
Theorem 7.7 (Stability Lemma). Assume $\tilde{u}$ is a near solution on $I \times \mathbb{R}^{d}$

$$
\partial_{t}^{2} \tilde{u}-\Delta \tilde{u}=F(\tilde{u})+e
$$

such that

(i) Finite energy Strichartz solution:

$$
\left\|\left(\tilde{u}, \partial_{t} \tilde{u}\right)\right\|_{L_{t}^{\infty}\left(I ; \dot{H}^{1} \times L^{2}\right)}+\left\||\nabla|^{\frac{1}{2}} \tilde{u}\right\|_{L_{t, x}^{\frac{2(d+1)}{d-1}}} \leq E .
$$

(ii) The finite energy of difference of initial data:

$$
\left\|\left(\tilde{u}_{0}-u_{0}, \tilde{u}_{1}-u_{1}\right)\right\|_{\dot{H}^{1} \times L^{2}} \leq E^{\prime} .
$$

(iii) Smallness perturbation:

$$
\begin{array}{r}
\left\|S(t)\left(\tilde{u}_{0}-u_{0}, \tilde{u}_{1}-u_{1}\right)\right\|_{S(I)} \leq \varepsilon \\
\left\||\nabla|^{\frac{1}{2}} e\right\|_{L_{t, x}^{\frac{2(d+1)}{d+3}}} \leq \varepsilon .
\end{array}
$$

Then there exists $\varepsilon_{0}=\varepsilon_{0}\left(d, E, E^{\prime}\right)$ such that if $\varepsilon \in\left(0, \varepsilon_{0}\right)$, for (FNLW) with initial data $\left(u_{0}, u_{1}\right)$, there exists a unique solution $u$ on $I \times \mathbb{R}^{d}$ with the properties

$$
\begin{aligned}
\|\tilde{u}-u\|_{S(I)} & \leq C\left(d, E, E^{\prime}\right) \cdot \varepsilon^{c}, \\
\|\tilde{u}-u\|_{\dot{S}^{1}(I)} & \leq C\left(d, E, E^{\prime}\right) \cdot E^{\prime},
\end{aligned}
$$

where $c$ satisfies: when $d \in\{3,4,5\}, c=1$; when $d \geq 6, c=c(d) \in(0,1)$; and

$$
\|u\|_{\dot{S}^{1}(I)} \triangleq \sup _{(q, r) \in \Lambda}\left\{\|u\|_{L_{t}^{q} \dot{B}_{r, 2}^{1-\beta(r)}\left(I \times \mathbb{R}^{d}\right)}+\left\|\partial_{t} u\right\|_{L_{t}^{q} \dot{B}_{r, 2}^{-\beta(r)}\left(I \times \mathbb{R}^{d}\right)}\right\},
$$

where $\beta(r)=\frac{d+1}{2}\left(\frac{1}{2}-\frac{1}{r}\right)$,

$$
\Lambda:=\left\{(q, r): \frac{2}{q}=(d-1)\left(\frac{1}{2}-\frac{1}{r}\right), q, r \geq 2,(q, r, d) \neq(2, \infty, 3)\right\} .
$$

\subsection{Variational estimates.}

Lemma 7.8 (Coercivity Lemma). Assume that $E\left(v_{1}, v_{2}\right) \leq\left(1-\delta_{0}\right) E(W), \delta_{0} \in$ $(0,1)$. Then

(1) If $\left\|\nabla v_{1}\right\|_{L^{2}}<\|\nabla W\|_{L^{2}}$, then there exists $\delta_{1}=\delta_{1}\left(\delta_{0}\right)>0$ such that

(i) $\left\|\nabla v_{1}\right\|_{L^{2}} \leq\left(1-\delta_{1}\right)\|\nabla W\|_{L^{2}}$;

(ii) $\int_{\mathbb{R}^{d}}\left(\|\left.\nabla v_{1}\right|^{2}-\left|v_{1}\right|^{\frac{2 d}{d-2}}\right) d x \geq \delta_{1}\left\|\nabla v_{1}\right\|_{L^{2}}^{2}$;

(iii) $\quad c\left\|\left(v_{1}, v_{2}\right)\right\|_{\dot{H}^{1} \times L^{2}}^{2} \leq E\left(v_{1}, v_{2}\right) \leq \frac{1}{2}\left\|\left(v_{1}, v_{2}\right)\right\|_{\dot{H}^{1} \times L^{2}}^{2}$.

(2) If $\left\|\nabla v_{1}\right\|_{L^{2}}>\|\nabla W\|_{L^{2}}$, then there exists $\delta_{2}=\delta_{2}\left(\delta_{0}\right)>0$ such that

$$
\left\|\nabla v_{1}\right\|_{L^{2}} \geq\left(1+\delta_{2}\right)\|\nabla W\|_{L^{2}} \text {. }
$$

Proof. Let

$$
f(y)=\frac{1}{2} y-\frac{C_{d}^{2^{*}}}{2^{*}} y^{2^{*} / 2}, \bar{y} \triangleq\left\|\nabla v_{1}\right\|_{2}^{2}, 2^{*} \triangleq \frac{2 d}{d-2},
$$

where $C_{d}$ is the sharp constant of Sobolev inequality

$$
\|g(x)\|_{2^{*}} \leq C_{d}\|\nabla g\|_{2}, \quad\left(\|W(x)\|_{2^{*}}=C_{d}\|\nabla W(x)\|_{2}\right) .
$$




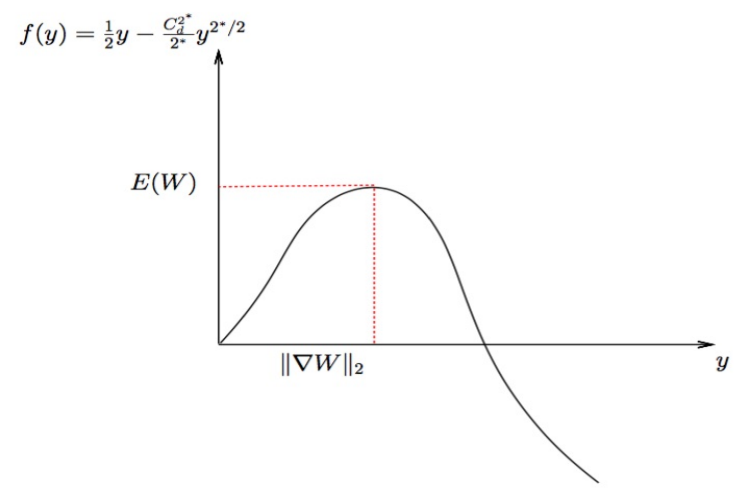

Note that $f(0)=0, f(y)>0$ for $y$ near $0, y>0$, and that

$$
f^{\prime}(y)=\frac{1}{2}-\frac{C_{d}^{2^{*}}}{2} y^{\frac{2^{*}}{2}-1},
$$

so that

$$
f^{\prime}(y)=0 \Longleftrightarrow y=y_{c}=\frac{1}{C_{d}^{d}}=\|\nabla W\|_{2}^{2} .
$$

Also

$$
\left\{\begin{array}{l}
f(\bar{y})=f\left(\left\|\nabla v_{1}\right\|^{2}\right) \leq E\left(v_{1}, v_{2}\right), \quad(\text { sharp Sobolev inequality) } \\
f\left(\|\nabla W\|_{2}^{2}\right)=f\left(y_{c}\right)=\frac{1}{d C_{d}^{d}}=E(W, 0) .
\end{array}\right.
$$

Since $f \geq 0$ is strictly increasing in $\left[0, y_{c}\right]=\left[0,\|\nabla W\|_{2}^{2}\right], f^{\prime \prime}\left(y_{c}\right)<0$, so $f$ arrive the maximal at $y=y_{c}=\|\nabla W\|_{2}^{2}$. Note that

$$
E\left(v_{1}, v_{2}\right)=\left(1-\delta_{0}\right) E(W, 0)<E(W, 0),
$$

there exists $\delta_{1}=\delta\left(\delta_{0}\right)$ such that

$$
f^{-1}\left(E\left(v_{1}, v_{2}\right)\right)=f^{-1}\left(\left(1-\delta_{0}\right) E(W, 0)\right)=\left(1-\delta_{1}\right)\|\nabla W\|_{2}^{2} .
$$

Hence

$$
\left\|\nabla v_{1}\right\|_{2}^{2}=\bar{y} \leq f^{-1}\left(E\left(v_{1}, v_{2}\right)\right)=f^{-1}\left(\left(1-\delta_{0}\right) E(W, 0)\right) \leq\left(1-\delta_{1}\right)\|\nabla W\|_{2}^{2} .
$$

This shows (1)(i). By the same way, we conclude (2).

For (1)(ii) note that

$$
\begin{aligned}
\int_{\mathbb{R}^{d}}\left(\left|\nabla v_{1}\right|^{2}-\left|v_{1}\right|^{2^{*}}\right) d x & \geq \int_{\mathbb{R}^{d}}\left|\nabla v_{1}\right|^{2} d x-C_{d}^{2^{*}}\left(\int_{\mathbb{R}^{d}}\left|\nabla v_{1}\right|^{2} d x\right)^{2^{*} / 2} \\
& =\left[1-C_{d}^{2^{*}}\left(\int_{\mathbb{R}^{d}}\left|\nabla v_{1}\right|^{2} d x\right)^{2 / d-2}\right] \int_{\mathbb{R}^{d}}\left|\nabla v_{1}\right|^{2} d x \\
& \geq\left[1-C_{d}^{2^{*}}\left(1-\delta_{1}\right)^{2 / d-2}\left(\int_{\mathbb{R}^{d}}|\nabla W|^{2} d x\right)^{2 / d-2}\right] \int_{\mathbb{R}^{d}}\left|\nabla v_{1}\right|^{2} d x \\
& =\left[1-\left(1-\delta_{1}\right)^{2 / d-2}\right] \int_{\mathbb{R}^{d}}\left|\nabla v_{1}\right|^{2} d x,
\end{aligned}
$$

which gives (1)(ii).

For (1)(iii), the upper bound is obviously, and the lower bound follows from (1)(ii). 
From this static Lemma, we obtain dynamic consequence.

Lemma 7.9 (Energy trapping). Let $u$ be a solution of (FNLW) with maximal interval $I$,

$$
E\left(u_{0}, u_{1}\right)<E(W, 0) .
$$

Choose $\delta_{0}>0$ such that $E\left(u_{0}, u_{1}\right) \leq\left(1-\delta_{0}\right) E(W, 0)$. Then, for $t \in I$, we have

(1) if $\left\|\nabla u_{0}\right\|_{2}<\|\nabla W\|_{2}$, then there exists $\delta_{1}=\delta_{1}\left(\delta_{0}\right)>0$ such that

(i) $\|\nabla u(t)\|_{2}^{2} \leq\left(1-\delta_{1}\right)\|\nabla W\|_{2}^{2}, E\left(u_{0}, u_{1}\right) \geq 0$;

(ii) $\int_{\mathbb{R}^{d}}\left(|\nabla u(t)|^{2}-|u(t)|^{2^{*}}\right) \geq \delta_{1} \int|\nabla u|^{2},($ "coercivity");

(iii) $E\left(u, \partial_{t} u\right) \simeq \delta_{0}\left\|\nabla\left(u(t), \partial_{t} u(t)\right)\right\|_{\dot{H}^{1} \times L^{2}}^{2} \simeq \delta_{0}\left\|\nabla\left(u_{0}, u_{1}\right)\right\|_{\dot{H}^{1} \times L^{2}}^{2}$.

(2) If $\left\|\nabla u_{0}\right\|_{L^{2}}>\|\nabla W\|_{L^{2}}$, then there exists $\delta_{2}=\delta_{2}\left(\delta_{0}\right)>0$ such that

$$
\|\nabla u(t)\|_{L^{2}} \geq\left(1+\delta_{2}\right)\|\nabla W\|_{L^{2}} .
$$

7.3. Proof of finite-time blowup. In this subsection, we prove (ii) in Theorem 2.5 .

Theorem 7.10 (Finite time blowup). Assume $\left(u_{0}, u_{1}\right) \in \dot{H}^{1} \times L^{2}$ with

$$
E\left(u_{0}, u_{1}\right)<E(W, 0) \text { and }\left\|\nabla u_{0}\right\|_{L^{2}}>\|\nabla W\|_{L^{2}} .
$$

Then, the solution $u$ to 2.9 blows up in finite time in both directions.

Proof. Without loss of generality, it suffices to consider $t>0$. By contradiction, we assume that the solution $u$ exists for all $t>0$.

Case 1: $u_{0} \in L^{2}\left(\mathbb{R}^{d}\right)$. By Lemma 7.9, we have

$$
\int_{\mathbb{R}^{d}}|\nabla u(t)|^{2} \geq\left(1+\delta_{1}\right) \int_{\mathbb{R}^{d}}|\nabla W|^{2}, t \in I
$$

and $E(W, 0) \geq E\left(u(t), \partial_{t} u\right)+\tilde{\delta}_{0}$ implies that

$$
\int_{\mathbb{R}^{d}}|u(t)|^{2^{*}} \geq \frac{d}{d-2} \int_{\mathbb{R}^{d}}\left|\partial_{t} u(t)\right|^{2}+\frac{d}{d-2} \int_{\mathbb{R}^{d}}|\nabla u(t)|^{2}-2^{*} E(W, 0)+2^{*} \tilde{\delta}_{0} .
$$

Let $y(t)=\int_{\mathbb{R}^{d}}|u(t)|^{2} d x$, so that

$$
y^{\prime}(t)=2 \int_{\mathbb{R}^{d}} u(t) \partial_{t} u(t) d x, y^{\prime \prime}(t)=2 \int_{\mathbb{R}^{d}}\left(\left|\partial_{t} u\right|^{2}-|\nabla u|^{2}+|u(t)|^{2^{*}}\right) d x .
$$

Thus,

$$
\begin{aligned}
y^{\prime \prime}(t) \geq & 2 \int_{\mathbb{R}^{d}}\left|\partial_{t} u\right|^{2} d x+\frac{2 d}{d-2} \int_{\mathbb{R}^{d}}\left|\partial_{t} u\right|^{2} d x-2 \cdot 2^{*} E(W, 0) \\
& +\tilde{\delta}_{0}+\frac{2 d}{d-2} \int_{\mathbb{R}^{d}}|\nabla u(t)|^{2} d x-2 \int_{\mathbb{R}^{d}}|\nabla u(t)|^{2} d x \\
= & \frac{4(d-1)}{d-2} \int_{\mathbb{R}^{d}}\left|\partial_{t} u\right|^{2} d x+\frac{4}{d-2}\left(\int_{\mathbb{R}^{d}}|\nabla u(t)|^{2} d x-\int_{\mathbb{R}^{d}}|\nabla W|^{2} d x\right)+\tilde{\delta}_{0} \\
\geq & \frac{4(d-1)}{d-2} \int_{\mathbb{R}^{d}}\left|\partial_{t} u\right|^{2} d x+\tilde{\delta}_{0} .
\end{aligned}
$$


If $I \cap[0,+\infty)=[0,+\infty)$, there exists $t_{0}>0$ so that

$$
y^{\prime}\left(t_{0}\right)>0, \quad y^{\prime}(t)>0, t>t_{0} .
$$

For $t>t_{0}$, we have

$$
y(t) y^{\prime \prime}(t) \geq \frac{4(d-1)}{d-2} \int\left|\partial_{t} u\right|^{2} \cdot \int|u|^{2} \geq \frac{d-1}{d-2} y^{\prime}(t)^{2},
$$

so that

$$
\frac{y^{\prime \prime}(t)}{y^{\prime}(t)} \geq \frac{d-1}{d-2} \frac{y^{\prime}(t)}{y(t)}
$$

or

$$
y^{\prime}(t) \geq C_{0} y(t)^{\frac{d-1}{d-2}}, \forall t>t_{0}
$$

This implies

$$
y(t)^{-\frac{1}{d-2}} \leq y\left(t_{0}\right)^{-\frac{1}{d-2}}-C_{0}\left(t-t_{0}\right) .
$$

Hence, for large $t, y(t)<0$ which contradicts with $y(t) \geq 0$.

Case 2: $u_{0} \in \dot{H}^{1}\left(\mathbb{R}^{d}\right)$. The proof follows by using, in addition, localization and finite speed of propagation.

Let $A=\left\|\left(u_{0}, u_{1}\right)\right\|_{\dot{H}^{1} \times L^{2}}>0$. From finite speed of propagation and local theory (see Lemma 7.15 below), there exists $\varepsilon_{0}>0$ such that, for $0<\varepsilon<\varepsilon_{0}$, there exists $M_{0}=M_{0}(\varepsilon)$, with

$$
\int_{|x| \geq M_{0}+t}\left(|\nabla u(x, t)|^{2}+\left|\partial_{t} u(x, t)\right|^{2}+|u(x, t)|^{2^{*}}+\frac{|u(x, t)|^{2}}{|x|^{2}}\right) d x \leq \varepsilon,
$$

for $t \in\left[0, T_{+}\left(u_{0}, u_{1}\right)\right)$. Assume that $T_{+}\left(u_{0}, u_{1}\right)=+\infty$ to reach a contradiction.

Let $f(\tau)$ be a solution to the differential inequality $(f \geq 0)$

$$
f^{\prime}(\tau) \geq B f(\tau)^{\frac{d-1}{d-2}}, \quad f(0)=1
$$

Then, the time of blow-up for $f$ is $\tau_{*}$, with $\tau_{*} \leq K_{d} B^{-1}$.

Let $\phi \in C_{0}^{\infty}\left(B_{2}\right)$ such that $\phi \equiv 1$ on $|x|<1,0 \leq \phi \leq 1$. Consider now, for $R$ large,

$$
y_{R}(t)=\int u^{2}(x, t) \phi(x / R) d x .
$$

Then, $y_{R}^{\prime}(t)=2 \int_{\mathbb{R}^{d}} u \partial_{t} u \phi(x / R) d x$, and

$$
y_{R}^{\prime \prime}(t)=2\left[\int_{\mathbb{R}^{d}}\left(\left|\partial_{t} u\right|^{2}-\left|\nabla_{x} u\right|^{2}+|u|^{2^{*}}\right) d x\right]+O(r(R)),
$$

with

$$
r(R)=\int_{|x| \geq R}\left(\frac{|u|^{2}}{|x|^{2}}+|u|^{2^{*}}+|\nabla u|^{2}+\left|\partial_{t} u\right|^{2}\right) d x .
$$

Arguing as in the proof of case 1 , we find that

$$
\left.y_{R}^{\prime \prime}(t) \geq \frac{4(d-1)}{d-2} \int_{\mathbb{R}^{d}} \mid \partial_{t} u\right)\left.\right|^{2} \phi(x / R) d x+\widetilde{\widetilde{\delta}}_{0}+O(r(R)) .
$$


Choose now $\varepsilon_{1}$ so small, and $M_{0}=M_{0}\left(\varepsilon_{1}\right)$, as above, so that, for $R>M_{0} / 2$, $O(r(R)) \leq \varepsilon_{1}, \varepsilon_{1} \leq \widetilde{\widetilde{\delta}}_{0} / 2$. We then have, for $0<t<R / 2$,

$$
\left\{\begin{array}{l}
y_{R}^{\prime \prime}(t) \geq \widetilde{\widetilde{\delta}}_{0} / 2, \\
y_{R}^{\prime \prime}(t) \geq \frac{4(d-1)}{d-2} \int_{\mathbb{R}^{d}}\left|\partial_{t} u\right|^{2} \phi(x / R) d x .
\end{array}\right.
$$

It is easy to verify that

$$
y_{R}(0) \leq C M_{0}^{2} A^{2}+\varepsilon_{1} R^{2}, \quad\left|y_{R}^{\prime}(0)\right| \leq C M_{0} A^{2}+\varepsilon_{1} R,
$$

where $A=\left\|\left(u_{0}, u_{1}\right)\right\|_{\dot{H}^{1} \times L^{2}}$. Let

$$
T=\frac{4 C M_{0} A^{2}+2 \varepsilon_{1} R+2 R \sqrt{\varepsilon_{1}}}{\widetilde{\widetilde{\delta}}_{0}},
$$

then, (if $T<R / 2$ )

$$
\begin{aligned}
y_{R}^{\prime}(T) & \geq T \frac{\widetilde{\tilde{\delta}}_{0}}{2}+y_{R}^{\prime}(0) \geq 2 C M_{0} A^{2}+\varepsilon_{1} R+R \sqrt{\varepsilon_{1}}-C M_{0} A^{2}-\varepsilon_{1} R \\
& =C M_{0} A^{2}+R \sqrt{\varepsilon_{1}} .
\end{aligned}
$$

Thus, there exists $0<t_{1}<T$ such that $y_{R}^{\prime}\left(t_{1}\right)=C M_{0} A^{2}+R \sqrt{\varepsilon_{1}}$, and for $0<$ $t<t_{1}$, we have $y_{R}^{\prime}(t)<C M_{0} A^{2}+R \sqrt{\varepsilon_{1}}$. Note that, in light of $7.20, y_{R}^{\prime}(t)>$ $y_{R}^{\prime}\left(t_{1}\right)>0, t>t_{1}(t<R / 2)$ and also

$y_{R}\left(t_{1}\right) \leq y_{R}(0)+\int_{0}^{t_{1}} y_{R}^{\prime}(\tau) d \tau \leq y_{R}(0)+t_{1}\left(C M_{0} A^{2}+R \sqrt{\varepsilon_{1}}\right)=y_{R}(0)+t_{1} y_{R}^{\prime}\left(t_{1}\right)$.

For sufficient small $\varepsilon_{1}$, we claim

$$
T=\frac{4 C M_{0} A^{2}}{\widetilde{\widetilde{\delta}}_{0}}+\frac{2 \varepsilon_{1} R}{\widetilde{\widetilde{\delta}}_{0}}+\frac{2 \sqrt{\varepsilon_{1}} R}{\widetilde{\widetilde{\delta}}_{0}} \leq \frac{1}{8 K_{d}} R
$$

In fact, we first choose $\varepsilon_{1}$ so small that $\frac{2 \varepsilon_{1}}{\widetilde{\delta}_{0}}+\frac{2 \sqrt{\varepsilon_{1}}}{\widetilde{\delta}_{0}} \leq \frac{1}{32 K_{d}}$, where $K_{d}$ is the constant defined at the beginning of the proof, and $R$ so large that $\frac{4 C M_{0} A^{2}}{\widetilde{\delta}_{0}} \leq \frac{1}{16 K_{d}} R$. We then prove the claim, and also ensure $T \leq \frac{R}{8}$. Thus,

$$
y_{R}\left(t_{1}\right) \leq C M_{0}^{2} A^{2}+\varepsilon_{1} R^{2}+\frac{R}{8 K_{d}} y_{R}^{\prime}\left(t_{1}\right) .
$$

If we now use the argument in the proof of Case 1, for the function

$$
\widetilde{y}_{R}(\tau)=y_{R}\left(t_{1}+\tau\right), \quad 0 \leq \tau \leq R / 4,
$$

in light of $(7.20)$, we see that, for $0<\tau<R / 4$, we have

$$
\left(\log \widetilde{y}_{R}^{\prime}(\tau)\right)^{\prime} \geq\left(\frac{(d-1)}{(d-2)} \log \widetilde{y}_{R}(\tau)\right)^{\prime} .
$$

So that, by integration,

$$
\frac{\widetilde{y}_{R}^{\prime}(\tau)}{\widetilde{y}_{R}^{\prime}(0)} \geq\left[\frac{\widetilde{y}_{R}(\tau)}{\widetilde{y}_{R}(0)}\right]^{\frac{(d-1)}{(d-2)}} \quad \text { for } 0 \leq \tau \leq R / 4 .
$$


Thus, if $f(\tau)=\frac{\widetilde{y}_{R}(\tau)}{\widetilde{y}_{R}(0)}$ and $B=\frac{\widetilde{y}_{R}^{\prime}(0)}{\widetilde{y}_{R}(0)}=\frac{y_{R}^{\prime}\left(t_{1}\right)}{y_{R}\left(t_{1}\right)}$, we have that $f$ is a solution of 7.19 for $0 \leq \tau \leq R / 4$. Thus, we must have

$$
\frac{R}{4} \leq \frac{y_{R}\left(t_{1}\right)}{y_{R}^{\prime}\left(t_{1}\right)} K_{d} \leq \frac{K_{d}\left(C M_{0}^{2} A^{2}+\varepsilon_{1} R^{2}\right)}{y_{R}^{\prime}\left(t_{1}\right)}+\frac{R}{8},
$$

or

$$
\begin{aligned}
\frac{1}{8} & \leq \frac{K_{d}\left(C M_{0}^{2} A^{2}+\varepsilon_{1} R^{2}\right)}{C M_{0} A^{2} R+\sqrt{\varepsilon_{1}} R^{2}}=\frac{K_{d}\left[C M_{0}^{2} A^{2} / R^{2}+\varepsilon_{1}\right]}{\left[C M_{0} A^{2} / R+\sqrt{\varepsilon_{1}}\right]} \\
& \leq K_{d} M_{0} / R+K_{d} \sqrt{\varepsilon_{1}} .
\end{aligned}
$$

By taking $K_{d} \sqrt{\varepsilon_{1}}<\frac{1}{32}$, and $\frac{K_{d} M_{0}}{R}<\frac{1}{32}$ we reach a contradiction, which gives the proof of Theorem 2.5 (ii).

7.4. Concentration-compactness procedure. We proceed "concentration-compactness" in dispersive framework developed by Kenig-Merle with profile decomposition of Bahouri-Gérard [3] for $d=3$ and Bulut 9 for $d \geq 4$. Thus, arguing by contradiction, we find a number $E_{c}$, with $0<\eta_{0} \leq E_{c}<E(W, 0)$ with the property that if

$$
E\left(u_{0}, u_{1}\right)<E_{c}, \quad\left\|\nabla u_{0}\right\|_{2}<\|\nabla W\|_{2},
$$

then $\|u\|_{S(I)}<\infty$. And $E_{c}$ is optimal with this property. We will see that this leads to a contradiction. We have:

Proposition 7.11 (Critical element). There exists

$$
\left(u_{0, c}, u_{1, c}\right) \in \dot{H}^{1} \times L^{2}, \quad\left\|\nabla u_{0, c}\right\|_{2}<\|\nabla W\|_{2}, E\left(u_{0, c}, u_{1, c}\right)=E_{c},
$$

such that for the corresponding solution $u_{c}$, we have

$$
\left\|u_{c}\right\|_{S(I)}=+\infty \text {. }
$$

Proposition 7.12 (Compactness). For any $u_{c}$ as in the above Proposition, with (say)

$$
\left\|u_{c}\right\|_{S\left(I_{+}\right)}=+\infty, \quad I+=I \cap[0,+\infty)
$$

there exist $x(t) \in \mathbb{R}^{d}, \lambda(t) \in \mathbb{R}^{+}, t \in I_{+}$, such that

$$
K=\left\{\vec{v}(x, t)=\left(\frac{1}{\lambda(t)^{\frac{d-2}{2}}} u\left(\frac{x-x(t)}{\lambda(t)}, t\right), \frac{1}{\lambda(t)^{\frac{d}{2}}} \partial_{t} u\left(\frac{x-x(t)}{\lambda(t)}, t\right)\right), t \in I_{+}\right\}
$$

has compact closure in $\dot{H}^{1} \times L^{2}$.

Remark 7.13. (1) Because of the continuity of $u(t) \in \dot{H}^{1}$ with respect to $t \in I$, we can construct $\lambda(t), x(t)$ continuous in $\left[0, T_{+}\left(u_{0, c}, u_{1, c}\right)\right)$ with $\lambda(t)>0$.

(2) Because of scaling and the compactness of $\bar{K}$ above, if $T_{+}\left(u_{0, c}, u_{1, c}\right)<\infty$, one always has that

$$
\lambda(t) \geq \frac{C_{0}(K)}{T_{+}\left(u_{0, c}, u_{1, c}\right)-t} .
$$

(3) If $T_{+}\left(u_{0, c}, u_{1, c}\right)=+\infty$, we can always find another (possibly different) critical element $v_{c}$ with a corresponding $\tilde{\lambda}(t)$ such that $\tilde{\lambda}(t) \geq A_{0}>0$ for $t \in\left[0, T_{+}\left(v_{0, c}, v_{1, c}\right)\right)$. (Again by compactness of $\bar{K}$. ) 
(4) One can use the "profile decomposition" to also show that there exists a nondecreasing function $g:\left(0, E_{c}\right] \rightarrow[0,+\infty)$ so that if

$$
\left\|\nabla u_{0}\right\|_{2}<\|\nabla W\|_{2} \text { and } E\left(u_{0, c},, u_{1, c}\right) \leq E_{c}-\eta
$$

then $\|u\|_{S(\mathbb{R})} \leq g(\eta)$.

Up to here, we have used, in this step, only variational argument and "general arguments". To proceed further we need to use specific features of (NLW) to establish fine properties of critical elements.

The first one is a consequence of the finite speed of propagation and the compactness of $\bar{K}$.

Lemma 7.14 (Compact support set). Let $u_{c}(t)$ be a critical element, with $T_{+}\left(u_{0, c}, u_{1, c}\right)<$ $+\infty$. (We can assume, by scaling, that $T_{+}\left(u_{0, c}, u_{1, c}\right)=1$.) Then there exists $\bar{x} \in \mathbb{R}^{d}$ such that

$$
\operatorname{supp}\left(u_{c}(\cdot, t), \partial_{t} u_{c}(\cdot, t)\right) \subset B(\bar{x}, 1-t), 0<t<1 .
$$

After translation, we can take $\bar{x}=0$.

In order to prove Lemma 7.14, we will need the following consequence of the finite speed of propagation:

Lemma 7.15. Let $\left\|\left(u_{0}, u_{1}\right)\right\|_{\dot{H}^{1} \times L^{2}} \leq A$. If for some $M>0$ and $0<\varepsilon<\varepsilon_{0}(A)$, we have

$$
\int_{|x| \geq M}\left(\left|\nabla u_{0}\right|^{2}+\left|u_{1}\right|^{2}+\frac{\left|u_{0}\right|^{2}}{|x|^{2}}\right) d x \leq \varepsilon, \quad t \in\left(0, T_{+}\left(u_{0, c}, u_{1, c}\right)\right) .
$$

Then we have

$$
\int_{|x| \geq \frac{3}{2} M+t}\left(|\nabla u(t)|^{2}+\left|\partial_{t} u(t)\right|^{2}+|u(t)|^{2^{*}}+\frac{|u(t)|^{2}}{|x|^{2}}\right) d x \leq C \varepsilon .
$$

Proof. Indeed, we choose $\psi_{M}(x) \in C_{c}^{\infty}\left(\mathbb{R}^{d}\right)$ such that

$$
\psi_{M}=\left\{\begin{array}{ll}
1 & |x| \geq \frac{3}{2} M, \\
0 & |x| \leq M,
\end{array} \quad\left(u_{0, M}, u_{1, M}\right) \triangleq\left(\psi_{M} u_{0}, \psi_{M} u_{1}\right) .\right.
$$

From our assumptions, we have

$$
\left\|\left(u_{0, M}, u_{1, M}\right)\right\|_{\dot{H}^{1} \times L^{2}} \leq C \varepsilon .
$$

If $C \varepsilon_{0}<\tilde{\delta}$, where $\tilde{\delta}$ is as in the "local Cauchy theory", the corresponding solution $u_{M}$ of (FNLW) has maximal interval $\mathbb{R}$ and

$$
\sup _{t \in \mathbb{R}}\left\|\left(u_{M}(t), \partial_{t} u_{M}(t)\right)\right\|_{\dot{H}^{1} \times L^{2}} \leq 2 C \varepsilon .
$$

But, by finite speed of propagation,

$$
u_{M} \equiv u,|x| \geq \frac{3}{2} M+t, t \in\left[0, T_{+}\left(u_{0}, u_{1}\right)\right),
$$

this proves Lemma 7.15 .

Proof of Lemma 7.14. Step 1: Since $\lambda(t) \geq C_{0}(K) /(1-t)$, we claim that, for any $R_{0}>0$,

$$
\lim _{t \nearrow 1} \int_{\left|x+\frac{x(t)}{\lambda(t)}\right| \geq R_{0}}\left(\left|\nabla u_{c}(x, t)\right|^{2}+\left|\partial_{t} u_{c}(x, t)\right|^{2}+\frac{\left|u_{c}(x, t)\right|^{2}}{|x|^{2}}\right) d x=0 .
$$


Indeed, if

$$
\vec{v}(x, t)=\left(\frac{1}{\lambda(t)^{\frac{d-2}{2}}} u_{c}\left(\frac{x-x(t)}{\lambda(t)}, t\right), \frac{1}{\lambda(t)^{\frac{d}{2}}} \partial_{t} u_{c}\left(\frac{x-x(t)}{\lambda(t)}, t\right)\right),
$$

then, by the compactness of $\bar{K}$ and the fact that $\lambda(t) \rightarrow+\infty$ (as $t \rightarrow 1$ ),

$$
\int_{\left|x+\frac{x(t)}{\lambda(t)}\right| \geq R_{0}}\left(\left|\nabla u_{c}(x, t)\right|^{2}+\left|\partial_{t} u_{c}(x, t)\right|^{2}\right) d x=\int_{|y| \geq \lambda(t) R_{0}}|\vec{v}(y, t)|^{2} d y \rightarrow 0, t \rightarrow 1 .
$$

Because of this fact, using Lemma 7.15 with respect to backward in time, we have, for each $s \in[0,1), R_{0}>0$,

$$
\lim _{t \nearrow 1} \int_{\left|x+\frac{x(t)}{\lambda(t)}\right| \geq \frac{3}{2} R_{0}+(t-s)}\left(\left|\nabla u_{c}(x, s)\right|^{2}+\left|\partial_{t} u_{c}(x, s)\right|^{2}\right) d x=0 .
$$

Step 2: We next show that

$$
\frac{|x(t)|}{\lambda(t)} \leq M, t \in[0,1)
$$

If not, we can find $t_{n} \uparrow 1$, so that $\left|x\left(t_{n}\right)\right| / \lambda\left(t_{n}\right) \rightarrow+\infty$. Then, for all $R>0$,

$$
\{|x| \leq R\} \subset\left\{\frac{\left|x+x\left(t_{n}\right)\right|}{\lambda\left(t_{n}\right)} \geq \frac{3}{2} R_{0}+t_{n}\right\} \text { for } n \text { large, }
$$

so that, passing to the limit in $n$, for $s=0$, we obtain

$$
\int_{|x| \leq R}\left(\left|\nabla u_{0, c}\right|^{2}+\left|u_{1, c}\right|^{2}\right) d x=0
$$

a contradiction.

Step 3: Finally, pick $t_{n} \uparrow 1$ so that $x\left(t_{n}\right) / \lambda\left(t_{n}\right) \rightarrow-\bar{x}$. Observe that, for every $\eta_{0}>0$, for $n$ large enough, for all $s \in[0,1)$,

$$
\left\{|x-\bar{x}| \geq 1+\eta_{0}-s\right\} \subset\left\{\left|x+\frac{x\left(t_{n}\right)}{\lambda\left(t_{n}\right)}\right| \geq \frac{3}{2} R_{0}+\left(t_{n}-s\right)\right\}
$$

for some $R_{0}=R_{0}\left(\eta_{0}\right)>0$. From this we conclude that

$$
\int_{|x-\bar{x}| \geq 1+\eta_{0}-s}\left(|\nabla u(x, s)|^{2}+\left|\partial_{s} u(x, s)\right|^{2}\right) d x=0,
$$

which gives the Lemma.

7.5. Zero momentum of critical element. We next turn to a result which is fundamental for us to be able to treat non-radial data.

Theorem 7.16 (Zero momentum: orthogonality for critical elements). Let ( $\left.u_{0, c}, u_{1, c}\right)$ be as in Proposition 7.11, with $\lambda(t), x(t)$ continuous. Assume that

either $T_{+}\left(u_{0, c}, u_{1, c}\right)<\infty \quad$ or $T_{+}\left(u_{0, c}, u_{1, c}\right)=\infty, \lambda(t) \geq A_{0}>0$.

Then

$$
\int_{\mathbb{R}^{d}} \nabla u_{0, c} \cdot u_{1, c} d x=0 .
$$

Remark 7.17. Note that in the radial case this is automatic by Plancherel Theorem. For non-radial case, we need a further linear estimate. 
Lemma 7.18 (Trace Lemma). Let $w$ solve the linear wave equation

$$
\left\{\begin{array}{l}
w_{t t}-\Delta w=h \in L_{t}^{1} L_{x}^{2}, \\
\left(w(0), \partial_{t} w(0)\right)=\left(w_{0}, w_{1}\right) \in \dot{H}^{1}\left(\mathbb{R}^{d}\right) \times L^{2}\left(\mathbb{R}^{d}\right) .
\end{array}\right.
$$

Then, for $|a| \leq \frac{1}{4}$, we have

$$
\sup _{t}\left\|\nabla_{t, x} w\left(\frac{x_{1}-a t}{\sqrt{1-a^{2}}}, x^{\prime}, \frac{t-a x_{1}}{\sqrt{1-a^{2}}}\right)\right\|_{L^{2}\left(d x_{1} d x^{\prime}\right)} \leq C\left[\left\|\left(w_{0}, w_{1}\right)\right\|_{\dot{H}^{1} \times L^{2}}+\|h\|_{L_{t}^{1} L_{x}^{2}}\right] .
$$

Proof. Let $v(x, t)=U(t) f$ be given by $\hat{v}(\xi, t)=e^{i t|\xi|} \hat{f}(\xi)$, with $f \in L^{2}$. We will show that

$$
\sup _{t}\left\|v\left(\frac{x_{1}-a t}{\sqrt{1-a^{2}}}, x^{\prime}, \frac{t-a x_{1}}{\sqrt{1-a^{2}}}\right)\right\|_{L^{2}\left(d x_{1} d x^{\prime}\right)} \leq C\|f\|_{L^{2}},
$$

which easily implies the desired estimate. But

$$
\begin{aligned}
v(x, t) & =\int_{\mathbb{R}^{d}} e^{i x \cdot \xi} e^{i t|\xi|} \hat{f}(\xi) d \xi=\int_{\mathbb{R}^{d}} e^{i x_{1} \xi_{1}} e^{i t|\xi|} e^{i x^{\prime} \cdot \xi^{\prime}} \hat{f}(\xi) d \xi_{1} d \xi^{\prime} \\
& =\int_{\mathbb{R}^{d}} e^{i x_{1} \xi_{1}} e^{i t \sqrt{\xi_{1}^{2}+\left|\xi^{\prime}\right|^{2}}} e^{i x^{\prime} \cdot \xi^{\prime}} \hat{f}\left(\xi_{1}, \xi^{\prime}\right) d \xi_{1} d \xi^{\prime}
\end{aligned}
$$

so that

$$
\begin{aligned}
& v\left(\frac{x_{1}-a t}{\sqrt{1-a^{2}}}, x^{\prime}, \frac{t-a x_{1}}{\sqrt{1-a^{2}}}\right) \\
= & \int_{\mathbb{R}^{d}} e^{i\left(x_{1}-a t\right) \xi_{1} / \sqrt{1-a^{2}}} e^{i\left(t-a x_{1}\right) \sqrt{\xi_{1}^{2}+\left|\xi^{\prime}\right|^{2}} / \sqrt{1-a^{2}}} e^{i x^{\prime} \cdot \xi^{\prime}} \hat{f}(\xi) d \xi_{1} d \xi^{\prime} \\
= & \int_{\mathbb{R}^{d}} e^{i x_{1}\left(\xi_{1}-a|\xi|\right) / \sqrt{1-a^{2}}} e^{i x^{\prime} \cdot \xi^{\prime}} e^{-i a t \xi_{1} / \sqrt{1-a^{2}}} e^{i t|\xi| / \sqrt{1-a^{2}}} \hat{f}(\xi) d \xi_{1} d \xi^{\prime} \\
= & \int_{\mathbb{R}^{d}} e^{i x_{1}\left(\xi_{1}-a|\xi|\right) / \sqrt{1-a^{2}}} e^{i x^{\prime} \cdot \xi^{\prime}} \hat{g}_{t}(\xi) d \xi_{1} d \xi^{\prime}
\end{aligned}
$$

where $\hat{g}_{t}(\xi)=e^{-i a t \xi_{1} / \sqrt{1-a^{2}}} e^{i t|\xi| / \sqrt{1-a^{2}}} \hat{f}(\xi)$. We now define

$$
\eta_{1}=\frac{\xi_{1}-a|\xi|}{\sqrt{1-a^{2}}}, \quad \eta^{\prime}=\xi^{\prime}
$$

and compute

$$
\begin{aligned}
\left|\frac{d \eta}{d \xi}\right| & =\operatorname{det}\left(\begin{array}{ccccc}
\frac{1-a \xi_{1} /|\xi|}{\sqrt{1-a^{2}}} \frac{-a \xi_{2} /|\xi|}{\sqrt{1-a^{2}}} & \ldots & \ldots & \frac{-a \xi_{d} /|\xi|}{\sqrt{1-a^{2}}} & \\
0 & 1 & 0 & \cdots & 0 \\
0 & 0 & 1 & \ldots & 0 \\
\vdots & \vdots & \vdots & \ddots & \vdots \\
0 & 0 & 0 & \ldots & 1
\end{array}\right) \\
& =\left(\frac{1-a \xi_{1} /|\xi|}{\sqrt{1-a^{2}}}\right) \approx 1 \text { for }|a| \leq 1 / 4 .
\end{aligned}
$$

The result now follows from Plancherel's Theorem.

Remark 7.19. A density argument in fact shows that

$$
t \mapsto w\left(\frac{x_{1}-a t}{\sqrt{1-a^{2}}}, x^{\prime}, \frac{t-a x_{1}}{\sqrt{1-a^{2}}}\right) \in C\left(\mathbb{R} ; \dot{H}^{1}\left(\mathbb{R}^{d}, d x_{1}, d \bar{x}^{\prime}\right)\right),
$$


and similarly for $\partial_{t} w$.

Note that if $u$ is a solution of (FNLW), with maximal interval $I$ and $I^{\prime} \subset \subset$ $I, u \in L_{I^{\prime}}^{\frac{d+2}{d-2}} L_{x}^{\frac{2(d+2)}{d-2}}$, and so $|u|^{\frac{4}{d-2}} u \in L_{I^{\prime}}^{1} L_{x}^{2}$. Thus, the conclusion of the Lemma 7.18 applies, provided the integration is restricted to

$$
\left(\frac{t-a x_{1}}{\sqrt{1-a^{2}}}, \frac{x_{1}-a t}{\sqrt{1-a^{2}}}, x^{\prime}\right) \in I^{\prime} \times \mathbb{R}^{d} .
$$

\section{The proof of Theorem 7.16}

Case 1: $T_{+}\left(u_{0, c}, u_{1, c}\right)<+\infty$. By scaling, we may assume $T_{+}\left(u_{0, c}, u_{1, c}\right)=1$. By Lemma 7.14, we have in this situation,

$$
\operatorname{supp}\left(u_{c}(\cdot, t), \partial_{t} u_{c}(\cdot, t)\right) \subset B(0,1-t), 0<t<1,
$$

Assume, to argue by contradiction, that

$$
\int_{\mathbb{R}^{d}} \partial_{x_{1}}\left(u_{0, c}\right) u_{1, c} d x=\gamma>0
$$

This equality will leads to a contradiction. For convenience, set

$$
u(x, t)=u_{c}(x, 1+t),-1<t<0,
$$

which satisfies the following properties

$$
\left\{\begin{array}{l}
\operatorname{supp} u(t, \cdot) \subset B(0,|t|), \quad \gamma=\int_{\mathbb{R}^{d}} \partial_{x_{1}} u(t) u_{t}(t) d x, \quad t \in[-1,0) ; \\
E\left(u(t), \partial_{t} u(t)\right)=E_{c}, \quad \int_{\mathbb{R}^{d}}|\nabla u(t)|^{2} d x \leq(1-\bar{\delta})\|\nabla W\|_{2}^{2}, \quad t \in[-1,0),
\end{array}\right.
$$

by energy trapping Lemma 7.9 . Note that

$$
\operatorname{supp} u(t, \cdot) \subset B(0,-t), \operatorname{supp} u_{t}(t, \cdot) \subset B(0,-t), \quad-1 \leq t<0,
$$

For $a \in(0,1 / 4)$, we consider the Lorentz transformation

$$
z_{a}\left(x_{1}, x^{\prime}, t\right)=u\left(\frac{x_{1}-a t}{\sqrt{1-a^{2}}}, x^{\prime}, \frac{t-a x_{1}}{\sqrt{1-a^{2}}}\right),
$$

where $x=\left(x_{1}, x^{\prime}\right) \in \mathbb{R}^{d}, t \in[-1,0)$ and $s=\left(t-a x_{1}\right) / \sqrt{1-a^{2}}$ is such that $-1 \leq s<0$.

Note that, for this range of $s$ and $y=\left(y_{1}, y^{\prime}\right) \operatorname{such} \operatorname{that}(y, s) \in \operatorname{supp} u$, we have $|y| \geq|s|$. Thus, if

$$
y_{1}=\frac{x_{1}-a t}{\sqrt{1-a^{2}}}, y^{\prime}=x^{\prime} \Longrightarrow\left\{\left.\left(x_{1}, x^{\prime}\right)\left|x_{1}^{2}+\right| x^{\prime}\right|^{2} \leq t\right\} \subset \operatorname{supp}\left(z_{a}, \dot{z}_{a}\right) .
$$

Fix now $t \in[-1 / 2,0)$ and $x_{1}^{2}+\left|x^{\prime}\right|^{2} \leq t^{2}$, we have

$$
0>\frac{(1-a) t}{\sqrt{1-a^{2}}} \geq \frac{t-a x_{1}}{\sqrt{1-a^{2}}} \geq \frac{(1+a) t}{\sqrt{1-a^{2}}} \geq-\frac{1}{2} \frac{1+a^{2}}{\sqrt{1-a^{2}}} \geq-1
$$

Hence, for such $(x, t), z_{a}$ is defined and satisfies

$$
z_{a}(x, t)=0, \quad \nabla z_{a}(x, t)=0, \quad \partial_{t} z_{a}(x, t)=0, \quad-\frac{1}{2} \leq t<0, x_{1}^{2}+\left|x^{\prime}\right|^{2}=t^{2} .
$$

We extend $z_{a}(\cdot, t)$ to be zero for $|x| \geq t,-\frac{1}{2} \leq t<0$. An elementary calculation shows that if $u$ is a regular solution to (NLW) in $\mathbb{R}^{d} \times[-1,0)$, then the resulting $z_{d}$ is in the energy space and solves our equation (NLW) in $\mathbb{R}^{d} \times[-1 / 2,0)$. 
The above easy calculation shows that

$$
\operatorname{supp} z_{a}(t, \cdot) \subset B(0,|t|), \quad z_{a} \not \equiv 0
$$

so that $T_{+}=0$ is the final time of existence for $z_{a}$. A lengthy calculation shows that

$$
\lim _{a \downarrow 0} \frac{E\left(z_{a}, \partial_{t} z_{a}\right)\left(-\frac{1}{2}, \cdot\right)-E\left(u_{0, c}, u_{1, c}\right)}{a}=-\int_{\mathbb{R}^{d}} \partial_{x_{1}}\left(u_{0, c}\right) u_{1, c} d x \triangleq-\gamma<0 .
$$

and for small a such that

$$
\left\{\begin{array}{l}
E\left(z_{a}, \partial_{t} z_{a}\right)\left(t_{0}, \cdot\right)<E\left(u_{0, c}, u_{1, c}\right)=E_{c}, \\
\int_{\mathbb{R}^{d}}\left|\nabla z_{a}\left(t_{0}\right)\right|^{2} d x<\int_{\mathbb{R}^{d}}|\nabla W|^{2} d x,
\end{array} \quad \text { for some } t_{0} \in\left[-\frac{1}{2},-\frac{1}{4}\right] .\right.
$$

But, for $a$ small the above facts impliy that $z_{a}$ should be global solution! This contradicts with the fact the final time of existence of $z_{a}$ is finite! and so contradicts with the definition of $E_{c}$ by taking $a>0$ small!

Case 2: $T_{+}\left(u_{0, c}, u_{1, c}\right)=+\infty$. In this case we have $\lambda(t) \geq A_{0}>0$. The finiteness of the energy of $z_{a}$ is unclear, because of the lack of the support property. We instead do a renormalization. For large $R$, we first rescale $u_{c}$ as $u_{R}(x, t)=R^{\frac{d-2}{2}} u_{c}(R x, R t)$, and for small $a$, define

$$
z_{a, R}\left(t, x_{1}, x^{\prime}\right) \triangleq u_{R}\left(\frac{t-a x_{1}}{\sqrt{1-a^{2}}}, \frac{x_{1}-a t}{\sqrt{1-a^{2}}}, x^{\prime}\right) .
$$

We assume that

$$
\int_{\mathbb{R}^{d}} \partial_{x_{1}}\left(u_{0, c}\right) u_{1, c} d x=\gamma>0
$$

and hope to obtain a contradiction. We can prove (by integration in $t_{0} \in(1,2)$ ) that if

$$
h\left(t_{0}\right)=\theta(x) z_{a, R}\left(t_{0}, x_{1}, x^{\prime}\right)
$$

with $\theta$ a fixed cut-off function, for some $a_{1}$ small and $R$ large, we have, for some $t_{0} \in(1,2)$, that

$$
E\left(h\left(t_{0}\right), \partial_{t} h\left(t_{0}\right)\right)<E_{c}-\frac{a_{1} \gamma}{2} \text { and } \int_{\mathbb{R}^{d}}\left|\nabla h\left(t_{0}\right)\right|^{2} d x<\int_{\mathbb{R}^{d}}|\nabla W|^{2} d x .
$$

We then let $v$ be the solution of (NLW) with data $h\left(t_{0}, \cdot\right)$, i.e.

$$
\left\{\begin{array}{l}
v_{t t}-\Delta v=-|v|^{\frac{4}{d-2}} v, \quad(t, x) \in \mathbb{R} \times \mathbb{R}^{d}, \\
v\left(t_{0}\right)=\theta(x) z_{a, R}\left(x, t_{0}\right), \quad v_{t}\left(t_{0}\right)=\theta(x) \dot{z}_{a, R}\left(x, t_{0}\right) .
\end{array}\right.
$$

By the properties of critical element, we know that

$$
\|v\|_{S(\mathbb{R})} \leq g\left(\frac{a_{1} \gamma}{2}\right)<\infty, \quad \text { for } R \text { large }
$$

On the other hand, since $\left\|u_{c}\right\|_{S(0,+\infty)}=+\infty$, we have that

$$
\left\|u_{R}\right\|_{L_{t, x} \frac{2(d+1)}{d-2}}^{([0,1] \times\{|x|<1\})} \rightarrow+\infty \text {, as } R \rightarrow \infty .
$$

But, by finite speed of propagation, we have that $v=z_{a, R}$ on a large set and, after a change of variables to undo the Lorentz transformation, we reach a contradiction from 7.28 and 7.29 . 
7.6. Rigidity theorem. To finish Theorem 2.5, we are reduced to:

Theorem 7.20 (Rigidity Theorem). Assume that $E\left(u_{0}, u_{1}\right)<E(W, 0)$, and $\left\|\nabla u_{0}\right\|_{2}<$ $\|\nabla W\|_{2}$. Let $u$ be the corresponding solution of (FNLW), and let $I_{+}=\left[0, T_{+}\left(u_{0}, u_{1}\right)\right)$. Suppose that:

(i) $\int_{\mathbb{R}^{d}} \nabla u_{0} u_{1} d x=0$.

(ii) There exist $x(t), \lambda(t)$ such that

$$
K=\left\{\vec{v}(x, t)=\left(\lambda(t)^{-\frac{d-2}{2}} u_{c}\left(\frac{x-x(t)}{\lambda(t)}, t\right), \lambda(t)^{-\frac{d}{2}} \partial_{t} u_{c}\left(\frac{x-x(t)}{\lambda(t)}, t\right)\right), t \in I_{+}\right\}
$$

has compact closure in $\dot{H}^{1} \times L^{2}$.

(iii) $x(t), \lambda(t)$ are continuous, $\lambda(t)>0$.

$$
\left\{\begin{array}{l}
\text { If } T_{+}\left(u_{0}, u_{1}\right)<\infty, \text { we have } \lambda(t) \geq \frac{C}{T_{+}-t}, \operatorname{supp}\left(u, \partial_{t} u\right) \subset B\left(0, T_{+}-t\right) ; \\
\text { if } T_{+}\left(u_{0}, u_{1}\right)=\infty, \text { we have } x(0)=0, \lambda(0)=1, \lambda(t) \geq A_{0}>0 .
\end{array}\right.
$$

Then $T_{+}<\infty$ is impossible, and if $T_{+}=\infty, \Longrightarrow u \equiv 0$.

Virial identity This Rigidity Theorem provides the contradiction for the proof of Theorem 2.5. For the proof we need some known identities (see [77, 125]).

Lemma 7.21 (Algebraic identity). Let

$$
r(R)=r(t, R)=\int_{|x| \geq R}\left(|\nabla u|^{2}+\left|\partial_{t} u\right|^{2}+|u|^{2^{*}}+\frac{|u|^{2}}{|x|^{2}}\right) d x .
$$

Let $u$ be a solution of (FNLW), $t \in I, \phi_{R}(x)=\phi(x / R), \psi_{R}(x)=x \phi(x / R)$, where $\phi \in C_{0}^{\infty}\left(B_{2}\right), \phi \equiv 1$ on $|x| \leq 1$. Then:

(i) $\partial_{t}\left(\int_{\mathbb{R}^{d}} \psi_{R} \nabla u \partial_{t} u d x\right)=-\frac{d}{2} \int_{\mathbb{R}^{d}}\left|\partial_{t} u\right|^{2} d x+\frac{d-2}{2} \int_{\mathbb{R}^{d}}\left(|\nabla u|^{2}-|u|^{2^{*}}\right) d x+O(r(R))$.

(ii) $\partial_{t}\left(\int_{\mathbb{R}^{d}} \phi_{R} u \partial_{t} u d x\right)=\int_{\mathbb{R}^{d}}\left|\partial_{t} u\right|^{2} d x-\int_{\mathbb{R}^{d}}|\nabla u|^{2} d x+\int_{\mathbb{R}^{d}}|u|^{2^{*}} d x+O(r(R))$.

(iii) $\partial_{t}\left(\int_{\mathbb{R}^{d}} \psi_{R}\left[\frac{1}{2}|\nabla u|^{2}+\frac{1}{2}\left|\partial_{t} u\right|^{2}-\frac{1}{2^{*}}|u|^{2^{*}}\right] d x\right)=-\int_{\mathbb{R}^{d}} \nabla u \partial_{t} u d x+O(r(R))$.

\section{The Proof of Rigidity theorem $\mathbf{7 . 2 0}$}

Case 1: $T_{+}\left(u_{0}, u_{1}\right)=+\infty$

We start out the proof of case 1 , without loss of generality, we may assume $x(t)$, $\lambda(t)$ continuous such that

$$
\lambda(t) \geq A_{0}>0, \quad x(0)=0, \quad \lambda(0)=1 .
$$

Assume that $\left(u_{0}, u_{1}\right) \neq(0,0)$ such that

$$
E=E\left(u_{0}, u_{1}\right) \leq\left(1-\delta_{0}\right) E(W, 0), \quad\left\|\nabla u_{0}\right\|_{2}<\|\nabla W\|_{2},
$$

then, from variational estimates,

$$
\sup _{t>0}\left\|\left(\nabla u(t), \partial_{t} u(t)\right)\right\|_{\dot{H}^{1} \times L^{2}} \leq C E, \quad E>0 .
$$


We also have

$$
\left\{\begin{array}{l}
\int_{\mathbb{R}^{d}}\left(|\nabla u(t)|^{2}-|u(t)|^{2^{*}}\right) d x \geq C_{\bar{\delta}} \int_{\mathbb{R}^{d}}|\nabla u(t)|^{2} d x, \quad \bar{\delta}=\bar{\delta}\left(\delta_{0}\right), \quad t>0 \\
\frac{1}{2} \int_{\mathbb{R}^{d}}\left|\partial_{t} u(t)\right|^{2} d x+\frac{1}{2} \int_{\mathbb{R}^{d}}\left(|\nabla u(t)|^{2}-|u(t)|^{2^{*}}\right) d x \geq C_{\bar{\delta}} E, \quad t>0 .
\end{array}\right.
$$

The compactness of $\bar{K}$ and the fact that $\lambda(t) \geq A_{0}>0$ show that, given $\epsilon>0$, we can find $R_{0}(\epsilon)>0$ so that, for all $t>0$, we have

$$
\int_{\left|x+\frac{x(t)}{\lambda(t)}\right| \geq R_{0}(\epsilon)}\left(|\nabla u|^{2}+\left|\partial_{t} u\right|^{2}+|u|^{2^{*}}+\frac{|u|^{2}}{|x|^{2}}\right) d x \leq \epsilon E .
$$

The proof of this case is accomplished through two lemmas.

Lemma 7.22 (Control-lemma). There exist $\epsilon_{1}, C>0$ such that, if $\epsilon \in\left(0, \epsilon_{1}\right), \exists R_{0}(\varepsilon)>$ 0 , for any $R>2 R_{0}(\epsilon)$, there exists $t_{0}=t_{0}(R, \epsilon)$ with $0<t_{0} \leq C R$, such that for $0<t<t_{0}$, we have

$$
\frac{|x(t)|}{\lambda(t)}<R-R_{0}(\epsilon) \text { and } \frac{\left|x\left(t_{0}\right)\right|}{\lambda\left(t_{0}\right)}=R-R_{0}(\epsilon)>R_{0}(\epsilon) .
$$

Note that in the radial case, since we can take $x(t) \equiv 0$, a contradiction follows directly from the above Lemma. This will be the analog of the local virial identity proof for the corresponding case of (NLS).

For the non-radial case we also need:

Lemma 7.23 ( $t_{0}$ control). There exists $\epsilon_{2} \in\left(0, \epsilon_{1}\right), R_{1}(\epsilon)>0, C_{0}>0$ so that if $R>R_{1}(\epsilon)$, for $0<\epsilon<\epsilon_{2}$, we have

$$
t_{0}(R, \epsilon) \geq C_{0} R / \epsilon
$$

From the above two Lemmas, we have, for

$$
\epsilon \in\left(0, \epsilon_{1}\right), \quad \Longrightarrow R>2 R_{0}(\epsilon), t_{0}(R, \epsilon) \leq C R,
$$

while for

$$
\epsilon \in\left(0, \epsilon_{2}\right), \quad \Longrightarrow R>R_{1}(\epsilon), t_{0}(R, \epsilon) \geq \frac{C_{0} R}{\epsilon} .
$$

Thus, for $\epsilon \in\left(0, \epsilon_{2}\right), R>\max \left\{R_{1}(\epsilon), 2 R_{0}(\epsilon)\right\}$, we have

$$
\frac{C_{0} R}{\epsilon} \leq t_{0}(R, \epsilon) \leq C R \text {. }
$$

This is clearly a contradiction for $\epsilon$ small. Therefore, $\left(u_{0}, u_{1}\right) \equiv 0$ in the case $T_{+}\left(u_{0}, u_{1}\right)=\infty$.

Thus, it suffices to show the above two Lemmas.

Proof of Lemma 7.22, If not, since $x(0)=0, \lambda(0)=1$, and both $\lambda(t), x(t)$ continuous, we have

$$
\forall t \in(0, C R) \text { with } C \text { large, } \frac{|x(t)|}{\lambda(t)}<R-R_{0}(\epsilon) .
$$

Let

$z_{R}(t)=\int_{\mathbb{R}^{d}}\left[\psi_{R} \nabla u \partial_{t} u+\frac{d-1}{2} \phi_{R} u \partial_{t} u\right] d x=\int_{\mathbb{R}^{d}}\left[\phi_{R}|x|\left(\frac{x}{|x|} \cdot \nabla+\frac{d-1}{2|x|}\right) u \partial_{t} u\right] d x$. 
Then

$$
z_{R}^{\prime}(t)=-\frac{1}{2} \int_{\mathbb{R}^{d}}\left|\partial_{t} u\right|^{2} d x-\frac{1}{2} \int_{\mathbb{R}^{d}}\left(|\nabla u|^{2}-|u|^{2^{*}}\right) d x+O(r(R)) .
$$

But, for $|x|>R, t \in(0, C R)$, we have by 7.33 and Lemma 7.9

$$
\left|x+\frac{x(t)}{\lambda(t)}\right| \geq R_{0}(\epsilon), \Longrightarrow|r(R)| \leq \epsilon E . \quad \Longrightarrow z_{R}^{\prime}(t) \leq-\tilde{C} E / 2, \text { for } \epsilon \text { small. }
$$

On the other hand, we have $\left|z_{R}(t)\right| \leq C_{1} R E$. Integrating in $t$ we obtain

$$
\frac{1}{2} C R \tilde{C} E \leq z_{R}(0)-z_{R}(C R) \leq 2 C_{1} R E, \Longrightarrow \text { a contradiction for } C \text { large. }
$$

Proof of Lemma 7.23: For $t \in\left[0, t_{0}\right]$, set

$$
y_{R}(t)=\int_{\mathbb{R}^{d}} \psi_{R}\left(\frac{1}{2}\left|\partial_{t} u\right|^{2}+\frac{1}{2}|\nabla u|^{2}-\frac{1}{2^{*}}|u|^{2^{*}}\right) d x, \quad \psi_{R}(x)=x \phi\left(\frac{x}{R}\right) .
$$

By Lemma 7.22 , we have

$$
\left|x+\frac{x(t)}{\lambda(t)}\right| \geq|x|-\left(R-R_{0}(\epsilon)\right) \geq R_{0}(\epsilon), \forall|x|>R .
$$

Conservation of momentum and (iii) in Lemma 7.21 imply that

$$
\int_{\mathbb{R}^{d}} \nabla u_{0} u_{1} d x=0=\int_{\mathbb{R}^{d}} \nabla u(t) \partial_{t} u(t) d x \text { and } y_{R}^{\prime}(t)=O(r(R)),
$$

and hence

$$
r(R) \leq E \epsilon, \Longrightarrow\left|y_{R}\left(t_{0}\right)-y_{R}(0)\right| \leq \tilde{C} \epsilon E t_{0} .
$$

However, noting that $x(0)=0$ and compactness 7.33 , we have

$$
\left|y_{R}(0)\right| \leq \tilde{C} R_{0}(\epsilon) E+O\left(R r\left(R_{0}(\epsilon)\right) \leq \tilde{C} E\left(R_{0}(\epsilon)+\epsilon R\right) .\right.
$$

Also

$$
\begin{aligned}
\left|y_{R}\left(t_{0}\right)\right| \geq & \left|\int_{\left|x+\frac{x\left(t_{0}\right)}{\lambda\left(t_{0}\right)}\right| \leq R_{0}(\epsilon)} \psi_{R}\left(\frac{1}{2}\left|\partial_{t} u\right|^{2}+\frac{1}{2}|\nabla u|^{2}-\frac{1}{2^{*}}|u|^{2^{*}}\right) d x\right| \\
& -\left|\int_{\left|x+\frac{x\left(t_{0}\right)}{\lambda\left(t_{0}\right)}\right|>R_{0}(\epsilon)} \psi_{R}\left(\frac{1}{2}\left|\partial_{t} u\right|^{2}+\frac{1}{2}|\nabla u|^{2}-\frac{1}{2^{*}}|u|^{2^{*}}\right) d x\right| .
\end{aligned}
$$

In the first integral, $|x| \leq R$ implies that $\psi_{R}(x)=x$. The second integral is bounded by $2 R \epsilon E$. Thus,

$$
\left|y_{R}\left(t_{0}\right)\right| \geq\left|\int_{\left|x+\frac{x\left(t_{0}\right)}{\lambda\left(t_{0}\right)}\right| \leq R_{0}(\epsilon)} x\left(\frac{1}{2}\left|\partial_{t} u\left(t_{0}\right)\right|^{2}+\frac{1}{2}\left|\nabla u\left(t_{0}\right)\right|^{2}-\frac{1}{2^{*}}\left|u\left(t_{0}\right)\right|^{2^{*}}\right) d x\right|-2 R \epsilon E .
$$

The integral on the right equals

$$
\begin{aligned}
& -\frac{x\left(t_{0}\right)}{\lambda\left(t_{0}\right)} \int_{\left|x+\frac{x\left(t_{0}\right)}{\lambda\left(t_{0}\right)}\right| \leq R_{0}(\epsilon)}\left(\frac{1}{2}\left|\partial_{t} u\left(t_{0}\right)\right|^{2}+\frac{1}{2}|\nabla u|^{2}-\frac{1}{2^{*}}\left|u\left(t_{0}\right)\right|^{2^{*}}\right) d x \\
+ & \int_{\left|x+\frac{x\left(t_{0}\right)}{\lambda\left(t_{0}\right)}\right| \leq R_{0}(\epsilon)}\left(x+\frac{x\left(t_{0}\right)}{\lambda\left(t_{0}\right)}\right) \cdot\left(\frac{1}{2}\left|\partial_{t} u\left(t_{0}\right)\right|^{2}+\frac{1}{2}\left|\nabla u\left(t_{0}\right)\right|^{2}-\frac{1}{2^{*}}\left|u\left(t_{0}\right)\right|^{2^{*}}\right) d x,
\end{aligned}
$$

so that its absolute value is greater than or equal to

$$
\left(R-R_{0}(\epsilon)\right) E-\tilde{C}\left(R-R_{0}(\epsilon)\right) \epsilon E-\tilde{C} R_{0}(\epsilon) E .
$$


Thus,

$$
\left|y_{R}\left(t_{0}\right)\right| \geq E\left(R-R_{0}(\epsilon)\right)(1-\tilde{C} \epsilon)-\tilde{C} R_{0}(\epsilon) E-2 R \epsilon E \geq E R / 4
$$

for $R$ large, $\epsilon$ small. But then, this together with 7.34 and 7.35 shows

$$
E R / 4-\tilde{C} E\left[R_{0}(\epsilon)+\epsilon R\right] \leq\left|y_{R}\left(t_{0}\right)\right|-\left|y_{R}(0)\right| \leq\left|y_{R}\left(t_{0}\right)-y_{R}(0)\right| \leq \tilde{C} \epsilon E t_{0},
$$

which yields the Lemma 7.23 for $\epsilon$ small, $R$ large.

Case 2: $T_{+}\left(u_{0}, u_{1}\right)<\infty$

We may assume $T_{+}\left(u_{0}, u_{1}\right)=1$. Then,

$$
\operatorname{supp}\left(u, \partial_{t} u\right)(t, \cdot) \subset B(0,1-t) \text { and } \lambda(t) \geq \frac{C}{1-t} .
$$

For (FNLW) we cannot use the conservation of the $L^{2}$-norm as in the (NLS) case and a new approach is needed. The first step is:

Lemma 7.24 (Upper bound of $\lambda(t)$ ). Let $u$ be as in the Rigidity Theorem 7.20, with $T_{+}\left(u_{0}, u_{1}\right)=1$. Then there exists $C>0$ so that

$$
\lambda(t) \leq \frac{C}{1-t}
$$

Remark: Thus, in this case

$$
\lambda(t) \simeq \frac{1}{1-t} .
$$

Proof. If not, we can find $t_{n} \nearrow 1$ so that $\lambda\left(t_{n}\right)\left(1-t_{n}\right) \rightarrow+\infty$.

Step 1: We claim that

$$
z(t) \geq C E \cdot(1-t), \quad t \in[0,1)
$$

where

$$
z(t)=\int x \cdot \nabla u \partial_{t} u+\frac{d-1}{2} \int u \partial_{t} u=\int|x|\left(\frac{x}{|x|} \cdot \nabla+\frac{d-1}{2|x|}\right) u \partial_{t} u .
$$

We recall that $z(t)$ is well-defined since

$$
\operatorname{supp}\left(u, \partial_{t} u\right)(t, \cdot) \subset B(0,1-t) .
$$

Then, for $t \in(0,1)$, by variational estimate 7.32 and $E\left(u_{0}, u_{1}\right)=E>0$, we have

$$
\left\{\begin{array}{l}
z^{\prime}(t)=-\frac{1}{2} \int_{\mathbb{R}^{d}}\left|\partial_{t} u\right|^{2} d x-\frac{1}{2} \int_{\mathbb{R}^{d}}\left(|\nabla u|^{2}-|u|^{2^{*}}\right) d x \leq-C E \\
\sup _{t \in(0,1)}\left\|\left(u(t), \partial_{t} u\right)\right\|_{\dot{H}^{1} \times L^{2}} \leq C E
\end{array}\right.
$$

From the support properties of $u$, it is easy to see that

$$
|z(t)| \leq(1-t)\left\|\left(u(t), \partial_{t} u(t)\right)\right\|_{\dot{H}^{1} \times L^{2}} \longrightarrow 0, \text { as } t \longrightarrow 1,
$$

so that, this together with 7.38 and integrating in $t$ gives

$$
z(t) \geq C E(1-t), t \in[0,1) .
$$

Step 2: We will next show that

$$
\frac{z\left(t_{n}\right)}{1-t_{n}} \rightarrow 0 \text { as } n \rightarrow \infty
$$

this yields a contradiction with the first step. 
Since

$$
\int_{\mathbb{R}^{d}} \nabla u(t) \partial_{t} u(t) d x=0
$$

we have

$$
\frac{z\left(t_{n}\right)}{1-t_{n}}=\int_{\mathbb{R}^{d}}\left(x+\frac{x\left(t_{n}\right)}{\lambda\left(t_{n}\right)}\right) \cdot \frac{\nabla u\left(t_{n}\right) \partial_{t} u\left(t_{n}\right)}{1-t_{n}} d x+\frac{d-1}{2} \int_{\mathbb{R}^{d}} \frac{u\left(t_{n}\right) \partial_{t} u\left(t_{n}\right)}{1-t_{n}} d x .
$$

Note that, for $\epsilon>0$ given, we have by compactness theorem

$$
\begin{aligned}
& \int_{\left|x+\frac{x\left(t_{n}\right)}{\lambda\left(t_{n}\right)}\right| \leq \epsilon\left(1-t_{n}\right)}\left[\left|x+\frac{x\left(t_{n}\right)}{\lambda\left(t_{n}\right)}\right| \cdot\left|\nabla u\left(t_{n}\right)\right| \cdot\left|\partial_{t} u\left(t_{n}\right)\right|+\left|u\left(t_{n}\right)\right| \cdot\left|\partial_{t} u\left(t_{n}\right)\right|\right] d x \\
\leq & C \epsilon E\left(1-t_{n}\right) .
\end{aligned}
$$

Now we consider the outsider region. First we will show that

$$
\frac{\left|x\left(t_{n}\right)\right|}{\lambda\left(t_{n}\right)} \leq 2\left(1-t_{n}\right)
$$

If not, $B\left(-\frac{x\left(t_{n}\right)}{\lambda\left(t_{n}\right)},\left(1-t_{n}\right)\right) \cap B\left(0,\left(1-t_{n}\right)\right)=\emptyset \Longrightarrow$

$$
\int_{B\left(-x\left(t_{n}\right) / \lambda\left(t_{n}\right),\left(1-t_{n}\right)\right)}\left(\left|\nabla u\left(t_{n}\right)\right|^{2}+\left|\partial_{t} u\left(t_{n}\right)\right|^{2}\right) d x \equiv 0
$$

while from the definition of compactness

$$
\begin{aligned}
& \int_{\left|x+\frac{x\left(t_{n}\right)}{\lambda\left(t_{n}\right)}\right| \geq\left(1-t_{n}\right)}\left(\left|\nabla u\left(t_{n}\right)\right|^{2}+\left|\partial_{t} u\left(t_{n}\right)\right|^{2}\right) d x \\
= & \int_{|y| \geq \lambda\left(t_{n}\right)\left(1-t_{n}\right)}\left|\nabla_{t, x} u\left(\frac{y-x\left(t_{n}\right)}{\lambda\left(t_{n}\right)}, t_{n}\right)\right|^{2} \frac{d y}{\lambda\left(t_{n}\right)^{d}} \longrightarrow 0, \text { as } n \longrightarrow \infty .
\end{aligned}
$$

This together with 7.40 contradicts $E>0$. Then, 7.39 and the Hardy inequality imply that

$$
\begin{aligned}
& \frac{1}{1-t_{n}} \int_{\left|x+\frac{x\left(t_{n}\right)}{\lambda\left(t_{n}\right)}\right| \geq \epsilon\left(1-t_{n}\right)}\left|x+\frac{x\left(t_{n}\right)}{\lambda\left(t_{n}\right)}\right| \cdot\left[\left|\nabla u\left(t_{n}\right)\right| \cdot\left|\partial_{t} u\left(t_{n}\right)\right|\right. \\
& \left.+\left|x+\frac{x\left(t_{n}\right)}{\lambda\left(t_{n}\right)}\right|^{-1}\left|u\left(t_{n}\right)\right| \cdot\left|\partial_{t} u\left(t_{n}\right)\right|\right] d x \\
\leq & 3 \int_{\left|x+\frac{x\left(t_{n}\right)}{\lambda\left(t_{n}\right)}\right| \geq \epsilon\left(1-t_{n}\right)}|| \nabla u\left(t_{n}\right)|\cdot| \partial_{t} u\left(t_{n}\right)|+| x+\left.\frac{x\left(t_{n}\right)}{\lambda\left(t_{n}\right)}\right|^{-1}\left|u\left(t_{n}\right)\right| \cdot\left|\partial_{t} u\left(t_{n}\right)\right| \mid d x \\
= & (3+C) \int_{|y| \geq \epsilon\left(1-t_{n}\right) \lambda\left(t_{n}\right)}\left(|\nabla u|^{2}+\left|\partial_{t} u\right|^{2}\right)\left(\frac{y-x\left(t_{n}\right)}{\lambda\left(t_{n}\right)}, t_{n}\right) \frac{d y}{\lambda\left(t_{n}\right)^{d}} \rightarrow 0
\end{aligned}
$$

by the compactness of $\bar{K}$ and the fact that $\lambda\left(t_{n}\right)\left(1-t_{n}\right) \rightarrow \infty$. And using Hardy's inequality (centered at $-x\left(t_{n}\right) / \lambda\left(t_{n}\right)$ ), the proof is concluded.

Proposition 7.25 (Self-similar solution). Let $u$ be as in the Rigidity Theorem 7.20. with $T_{+}\left(u_{0}, u_{1}\right)=1$,

$$
\operatorname{supp}\left(u, \partial_{t} u\right)(t, \cdot) \subset B(0,1-t)
$$

Then

$$
K=\left\{(1-t)^{\frac{d-2}{2}} u((1-t) x, t),(1-t)^{\frac{d}{2}} \partial_{t} u((1-t) x, t \in[0,1))\right\}
$$

is precompact in $\dot{H}^{1} \times L^{2}$. 
Proof. Note that the fact that if $\bar{K}_{1}$ is compact in $L^{2}\left(\mathbb{R}^{d}\right)^{d+1}$, then

$$
K_{2}:=\left\{\lambda^{\frac{d}{2}} \vec{v}(\lambda x): \vec{v} \in K_{1}, c_{0} \leq \lambda \leq c_{1}\right\}
$$

also has $\bar{K}_{2}$ compact. This together with the condition (iii) of Theorem 7.20 . combining this fact with $c_{0} \leq(1-t) \lambda(t) \leq c_{1}$, we get that the set

$$
\left\{\vec{v}(x, t)=(1-t)^{\frac{d}{2}}\left(\nabla u, \partial_{t} u\right)((1-t)(x-x(t)), t), t \in[0,1)\right\}
$$

has compact closure in $L^{2}\left(\mathbb{R}^{d}\right)^{d+1}$.

Let now

$$
\tilde{v}(x, t)=(1-t)^{\frac{d}{2}}\left(\nabla u, \partial_{t} u\right)((1-t) x, t),
$$

so that $\tilde{v}(x, t)=\vec{v}(x+x(t), t)$ satisfies

$$
\operatorname{supp} \vec{v}(\cdot, t) \subset\{x:|x-x(t)| \leq 1\} .
$$

The fact that $E>0$, the compactness of $\{\vec{v}(\cdot, t)\}$ and preservation of energy imply that $|x(t)| \leq M$. This together with (7.41) and the fact that if

$$
K_{3}=\left\{\vec{v}\left(x+x_{0}, t\right):\left|x_{0}\right| \leq M\right\},
$$

then $\overline{K_{3}}$ is compact, gives the Proposition.

\section{Self-similar variables and the end of proof of rigidity theorem}

Since the lack of the $L^{2}$ conservation law, we cannot use the conservation of the $L^{2}$-norm as in the (NLS). At this point we introduce a new idea, inspired by the works of Giga-Kohn[54] in the parabolic case and Merle-Zaag [103] in the hyperbolic case, who studied the equations

$$
\left(\partial_{t}^{2}-\Delta\right) u=|u|^{p-1} u, 1<p<1+\frac{4}{d-2},
$$

in the radial case. In our case, $p=1+\frac{4}{d-2}>1+\frac{4}{d-1}$. We thus introduce self-similar variables as following

$$
y=\frac{x}{1-t}, s=\log \frac{1}{1-t}, \quad \Longrightarrow \quad x=(1-t) y=e^{-s} y, t=1-e^{-s}
$$

and define

$$
w(y, s ; 0)=(1-t)^{\frac{d-2}{2}} u(x, t)=e^{-\frac{(d-2) s}{2}} u\left(e^{-s} y, 1-e^{-s}\right),
$$

which is defined for $0 \leq s<\infty$ with

$$
\operatorname{supp} w(\cdot, s ; 0) \subset\left\{y: e^{-s}|y| \leq e^{-s}\right\}=\{y:|y| \leq 1\} .
$$

We will also consider, for $\delta>0, u_{\delta}(x, t)=u(x, t+\delta)$ which also solves (FNLW) and its corresponding $w$, which we will denote by $w(y, s ; \delta)$. Thus, we set

$$
\left\{\begin{array}{l}
y=\frac{x}{1+\delta-t}, \quad s=\log \frac{1}{1+\delta-t}, \\
w(y, s ; \delta)=(1+\delta-t)^{\frac{d-2}{2}} u(x, t)=e^{-\frac{(d-2) s}{2}} u\left(e^{-s} y, 1+\delta-e^{-s}\right) .
\end{array}\right.
$$

Here $w(y, s ; \delta)$ is defined for $0 \leq s<-\log \delta$ and satisfies

$$
\operatorname{supp} w(\cdot, s ; \delta) \subset\left\{|y| \leq \frac{e^{-s}-\delta}{e^{-s}}=\frac{1-t}{1+\delta-t} \leq 1-\delta\right\} .
$$


Hence $w(y, s ; \delta)$ solves the equation in their domain

$$
\begin{aligned}
\partial_{s}^{2} w= & \frac{1}{\rho} \operatorname{div}(\rho \nabla w-\rho(y \cdot \nabla w) y)-\frac{d(d-2)}{4} w+|w|^{\frac{4}{d-2}} w \\
& -2 y \cdot \nabla \partial_{s} w-(d-1) \partial_{s} w, \quad \text { where } \rho(y)=\left(1-|y|^{2}\right)^{-1 / 2} .
\end{aligned}
$$

Note that the elliptic part of this operator degenerates. In fact,

$$
\frac{1}{\rho} \operatorname{div}(\rho \nabla w-\rho(y \cdot \nabla w) y)=\frac{1}{\rho} \operatorname{div}(\rho(I-y \otimes y) \nabla w),
$$

which is elliptic with smooth coefficients for $|y|<1$, but degenerates at $|y|=1$.

This new equation gives us a new set of formulas. The reason for introducing $\delta>0$ is that, on the $\operatorname{supp} w(\cdot, s, \delta)$, the inequality $\left(1-|y|^{2}\right) \geq \delta$ holds, so we stay away from the degeneracy. Now we collect some straightforward bounds on $w(\cdot, \cdot, \delta)$ with $\delta>0: \quad w \in H_{0}^{1}\left(B_{1}\right)$ with

$$
\int_{B_{1}}\left(|\nabla w|^{2}+\left|\partial_{s} w\right|^{2}+|w|^{2^{*}}\right) d x \leq C .
$$

Moreover, by Hardy's inequality for $H_{0}^{1}\left(B_{1}\right)$-functions, we have

$$
\int_{B_{1}} \frac{|w(y)|^{2}}{\left(1-|y|^{2}\right)^{2}} d y \leq C, \quad \text { uniformly in } \delta>0,0<s<-\log \delta .
$$

Next, following [103, we introduce an energy, which will provide us with a Lyapunov functional for $w$.

$$
\begin{aligned}
\tilde{E}(w(s ; \delta))= & \frac{1}{2} \int_{B_{1}}\left(|\nabla w|^{2}+\left|\partial_{s} w\right|^{2}-(y \cdot \nabla w)^{2}\right) \frac{d y}{\left(1-|y|^{2}\right)^{\frac{1}{2}}} \\
& +\int_{B_{1}}\left(\frac{d(d-2)}{8} w^{2}-\frac{d-2}{2 d}|w|^{2^{*}}\right) \frac{d y}{\left(1-|y|^{2}\right)^{\frac{1}{2}}}<\infty, \quad \forall \delta>0 .
\end{aligned}
$$

For $\delta>0$, we have the following new formulas:

Lemma 7.26. Let $0<s_{1}<s_{2}<\log 1 / \delta$, we have (i)

$$
\tilde{E}\left(w\left(s_{2}\right)\right)-\tilde{E}\left(w\left(s_{1}\right)\right)=\int_{s_{1}}^{s_{2}} \int_{B_{1}} \frac{\left|\partial_{s} w\right|^{2}}{\left(1-|y|^{2}\right)^{3 / 2}} d y d s, \quad \Longrightarrow \tilde{E} \nearrow .
$$

(ii)

$$
\begin{aligned}
& \left.\frac{1}{2} \int_{B_{1}}\left(\partial_{s} w\right) \cdot w-\frac{d+1}{2} w^{2}\right)\left.\frac{d y}{\left(1-|y|^{2}\right)^{1 / 2}}\right|_{s_{1}} ^{s_{2}} \\
= & -\int_{s_{1}}^{s_{2}} \tilde{E}(w(s)) d s+\frac{1}{d} \int_{s_{1}}^{s_{2}} \int_{B_{1}} \frac{|w|^{2^{*}}}{\left(1-|y|^{2}\right)^{1 / 2}} d s d y \\
& +\int_{s_{1}}^{s_{2}} \int_{B_{1}}\left(\left|\partial_{s} w\right|^{2}+\partial_{s} w \cdot(y \cdot \nabla) w+\frac{\partial_{s} w \cdot w|y|^{2}}{1-|y|^{2}}\right) \frac{d y}{\left(1-|y|^{2}\right)^{1 / 2}} .
\end{aligned}
$$

(iii) $\lim _{s \rightarrow \log 1 / \delta} \tilde{E}(w(s))=E\left(u_{0}, u_{1}\right)=E$, so that, by (i),

$$
-\frac{C}{\delta^{1 / 2}} \leq \tilde{E}(w(s)) \leq E \quad \text { for } \quad 0 \leq s<\log 1 / \delta .
$$




\section{The first improvement}

By (7.42) and the support property of $w$, we have

$$
\int_{0}^{1} \int_{B_{1}} \frac{\left|\partial_{s} w\right|^{2}}{1-|y|^{2}} d y d s \leq \frac{C}{\delta}
$$

Inspired by the above Lemma (i), we obtain:

Lemma 7.27 (First improvement).

$$
\int_{0}^{1} \int_{B_{1}} \frac{\left|\partial_{s} w\right|^{2}}{1-|y|^{2}} d y d s \leq C \log 1 / \delta
$$

Proof: Notice that

$$
\begin{aligned}
&-2 \int_{\mathbb{R}^{d}} \frac{\left|\partial_{s} w\right|^{2}}{1-|y|^{2}} d y= \frac{d}{d s}\left\{\int _ { \mathbb { R } ^ { d } } \left[\frac{1}{2}\left|\partial_{s} w\right|^{2}+\right.\right. \\
&-\frac{1}{2}\left(|\nabla w|^{2}-(y \cdot \nabla w)^{2}\right)+\frac{d(d-2)}{8} w^{2} \\
& \\
&+\int_{\mathbb{R}^{d}}\left[\log \left(1-|y|^{2}\right)+2\right] y \cdot \nabla w \partial_{s} w-\log \left(1-|y|^{2}\right)\left(\partial_{s} w\right)^{2} d y \\
&-2 \int_{\mathbb{R}^{d}}\left|\partial_{s} w\right|^{2} d y
\end{aligned}
$$

We next integrate in $s$, between 0 and 1 , and drop the term next to last term by sign. The proof is finished by using Cauchy-Schwartz and the support property of $w(\cdot, \delta)$.

\section{Corollary 7.28.}

$$
\begin{array}{ll}
\text { (a). } & \int_{0}^{1} \int_{B_{1}} \frac{|w|^{2^{*}}}{\left(1-|y|^{2}\right)^{1 / 2}} d y d s \leq C(\log 1 / \delta)^{\frac{1}{2}} \text {; } \\
\text { (b). } \quad \tilde{E}(w(1)) \geq-C(\log 1 / \delta)^{\frac{1}{2}} .
\end{array}
$$

Proof. Part (a) follows from Lemma 7.26(ii), (iii), Cauchy-Schwartz and Lemma 7.27. Note that we obtain the power $1 / 2$ on the right hand side by CauchySchwartz.

To prove Part (b), we first consider $\int_{0}^{1} \tilde{E}(w(s)) d s$, which is bounded from below by $-C(\log 1 / \delta)^{\frac{1}{2}}$ by Part (a). The monotonicity of $\tilde{E}(s)$ in Lemma 7.26)(i) shows

$$
\tilde{E}(w(1)) \geq \int_{0}^{1} \tilde{E}(w(s)) d s \geq-C(\log 1 / \delta)^{\frac{1}{2}} .
$$

\section{The second improvement}

Lemma 7.29 (Second improvement).

$$
\int_{1}^{\log 1 / \delta} \int_{B_{1}} \frac{\left|\partial_{s} w\right|^{2}}{\left(1-|y|^{2}\right)^{3 / 2}} d y d s \leq C(\log 1 / \delta)^{\frac{1}{2}} .
$$

Proof: Using Lemma 7.26 (i), (iii) and Corollary 7.28 (b), we have

$$
\int_{1}^{(\log 1 / \delta)^{3 / 4}} \int_{B_{1}} \frac{\left|\partial_{s} w\right|^{2}}{\left(1-|y|^{2}\right)^{3 / 2}} d y d s=\tilde{E}\left(w\left((\log 1 / \delta)^{3 / 4}\right)\right)-\tilde{E}(w(1)) \leq E+C(\log 1 / \delta)^{\frac{1}{2}}
$$


Corollary 7.30. There exists $\bar{s}_{\delta} \in\left(1,(\log 1 / \delta)^{3 / 4}\right)$ such that

$$
\int_{\bar{s}_{\delta}}^{\bar{s}_{\delta}+(\log 1 / \delta)^{1 / 8}} \int_{B_{1}} \frac{\left|\partial_{s} w\right|^{2}}{\left(1-|y|^{2}\right)^{3 / 2}} d y d s \leq \frac{C}{(\log 1 / \delta)^{1 / 8}} .
$$

Proof: Split $\left(1,(\log 1 / \delta)^{3 / 4}\right) \subset(1, \log 1 / \delta)$ into $(\log 1 / \delta)^{5 / 8}$ disjoint intervals of length $(\log 1 / \delta)^{1 / 8}$. Then Corollary follows from Lemma 7.29 and pigeonhole principle.

Remark 7.31. In Corollary 7.30, the length of the $s$ interval tends to infinity, while the bound goes to zero.

It is easy to see that if $\bar{s}_{\delta} \in\left(1,(\log 1 / \delta)^{3 / 4}\right)$ and $\bar{s}_{\delta}=-\log \left(1+\delta-\bar{t}_{\delta}\right)$, then

$$
\left|\frac{1-\bar{t}_{\delta}}{1+\delta-\bar{t}_{\delta}}-1\right| \leq C \delta^{\frac{1}{4}} \longrightarrow 0, \quad \text { as } \delta \longrightarrow 0 .
$$

From this and the compactness of $\bar{K}$, we can find $\delta_{j} \rightarrow 0$, so that

$$
w\left(y, \bar{s}_{\delta_{j}}+s ; \delta_{j}\right) \longrightarrow w^{*}(y, s) \in C\left([0, S] ; \dot{H}_{0}^{1} \times L^{2}\right), \text { for } s \in[0, S],
$$

and $w^{*}$ solves our self-similar equation in $B_{1} \times[0, S]$ as

$$
\begin{aligned}
\partial_{s}^{2} w= & \frac{1}{\rho} \operatorname{div}(\rho \nabla w-\rho(y \cdot \nabla w) y)-\frac{d(d-2)}{4} w+|w|^{\frac{4}{d-2}} w \\
& -2 y \cdot \nabla \partial_{s} w-(d-1) \partial_{s} w, \quad \text { where } \rho(y)=\left(1-|y|^{2}\right)^{-1 / 2} .
\end{aligned}
$$

The Corollary 7.30 shows that $w$ must be independent of $s$. Also, $E>0$ and our coercivity estimates show that $w^{*} \not \equiv 0$. Thus, $w^{*} \in H_{0}^{1}\left(B_{1}\right)$ solves the degenerate elliptic equation:

$$
\frac{1}{\rho} \operatorname{div}\left(\rho \nabla w^{*}-\rho\left(y \cdot \nabla w^{*}\right) y\right)-\frac{d(d-2)}{4} w^{*}+\left|w^{*}\right|^{\frac{4}{d-2}} w^{*}=0 .
$$

We next point out that $w^{*}$ satisfies the additional (crucial) estimates:

$$
\int_{B_{1}} \frac{\left|w^{*}\right|^{2^{*}}}{\left(1-|y|^{2}\right)^{1 / 2}} d y+\int_{B_{1}} \frac{\left|\nabla w^{*}\right|^{2}-\left(y \cdot \nabla w^{*}\right)^{2}}{\left(1-|y|^{2}\right)^{1 / 2}} d y<\infty .
$$

Indeed, for the first estimate it suffices to show that, uniformly for $j$ large, we have

$$
\int_{\bar{s}_{\delta_{j}}}^{\bar{s}_{\delta_{j}}+\delta} \int_{B_{1}} \frac{\left|w\left(y, s ; \delta_{j}\right)\right|^{2^{*}}}{\left(1-|y|^{2}\right)^{1 / 2}} d y d s \leq C .
$$

But this follows from (ii) above, together with the choice of $\bar{s}_{\delta_{j}}$, by the Corollary 7.30 Cauchy-Schwartz and (iii).

The proof of the second estimate follows from the first one, (iii) and the formula for $\tilde{E}$.

Remark 7.32. The conclusion of the proof is obtained by showing that a $w^{*}$ in $H_{0}^{1}\left(B_{1}\right)$, solving the degenerate elliptic equation with the additional bounds above, must be zero. This will follow from a unique continuation argument. 
Recall that, for $|y| \leq 1-\eta_{0}, \eta_{0}>0$, the linear operator is uniformly elliptic, with smooth coefficients and that the non-linearity is critical.

An argument going back to Trudinger's 142 shows that $w^{*}$ is bounded on $\{|y| \leq$ $\left.1-\eta_{0}\right\}$ for each $\eta_{0}>0$. Thus, if we show that $w^{*} \equiv 0$ near $|y|=1$, the standard Carleman unique continuation principle [63] will show that $w^{*} \equiv 0$.

Near $|y|=1$, our equation is modeled (in variables $(z, r) \in \mathbb{R}^{d-1} \times \mathbb{R}, r>0$, near $r=0$ ) by

$$
r^{\frac{1}{2}} \partial_{r}\left(r^{\frac{1}{2}} \partial_{r} w^{*}\right)+\Delta_{z} w^{*}+c w^{*}+\left|w^{*}\right|^{\frac{4}{d-2}} w^{*}=0
$$

Our information on $w$ translates into $w^{*} \in H_{0}^{1}((0,1] \times(|z|<1))$ and our crucial additional estimates are:

$$
\int_{0}^{1} \int_{|z|<1}\left|w^{*}(r, z)\right|^{2^{*}} \frac{d r}{r^{1 / 2}} d z+\int_{0}^{1} \int_{|z|<1}\left|\nabla_{z} w^{*}(r, z)\right|^{2} \frac{d r}{r^{1 / 2}} d z<\infty .
$$

We take advantage of the degeneracy of the equation. We "desingularize" the problem by letting $r=a^{2}$, setting $v(a, z)=w^{*}\left(a^{2}, z\right)$, so that

$$
\partial_{a} v(a, z)=2 a \partial_{r} w^{*}(r, z)=2 r^{\frac{1}{2}} \partial_{r} w^{*}(r, z) .
$$

Our equation becomes:

$$
\partial_{a}^{2} v+\Delta_{z} v+c v+|v|^{\frac{4}{d-2}} v=0,0<a<1,|z|<1,\left.v\right|_{a=0}=0
$$

and the bounds give:

$$
\left\{\begin{array}{l}
\int_{0}^{1} \int_{|z|<1}\left|\nabla_{z} v(a, z)\right|^{2} d a d z=\int_{0}^{1} \int_{|z|<1}\left|\nabla_{z} w^{*}(r, z)\right|^{2} \frac{d r}{r^{1 / 2}} d z<\infty \\
\int_{0}^{1} \int_{|z|<1}\left|\partial_{a} v(a, z)\right|^{2} \frac{d a}{a} d z=\int_{0}^{1} \int_{|z|<1}\left|\partial_{r} w^{*}(r, z)\right|^{2} d r d z<\infty
\end{array}\right.
$$

Thus, $v \in H_{0}^{1}\left((0,1] \times B_{1}\right)$, but from the additional bound we see that

$$
\left.\partial_{a} v(a, z)\right|_{a=0} \equiv 0 \text {. }
$$

We then extend $v$ by 0 to $a<0$ and see that the extension is an $H^{1}$ solution to the same equation. By Trudinger's argument, it is bounded.

But since it vanishes for $a<0$, by Carleman's unique continuation theorem, $v \equiv 0$. Hence, $w^{*} \equiv 0$, giving our contradiction.

\section{REFERENCES}

[1] T. Aubin. Équations différentielles non linéaires et problème de Yamabe concernant la courbure scalaire. J. Math. Pures Appl. (9), 55(3):269-296, 1976.

[2] H. Bahouri and J. Chemin, On global well-posedness for defocusing cubic wave equation, Internat. Math. Res. Notices, 2006: 54873.

[3] H. Bahouri, P. Gérard, High frequency approximation of solutions to critical nonlinear wave equations, Amer. J. Math., 121(1999), 131-175.

[4] P. Bizoń, Formation of singularities in Yang-Mills equations. Acta Phys. Polon. B 33, 7 (2002), 1893-1922.

[5] P. Bizoń, T. Chmaj, and Z. Tabor. On blowup for semilinear wave equations with a focusing nonlinearity. Nonlinearity, 17(6):2187-2201, 2004.

[6] J. Bourgain, Global well-posedness of defocusing 3D critical NLS in the radial case. J. Amer. Math. Soc., 12(1999), 145-171. 
[7] P. Brenner, P. Kumlin, On wave equations with supercritical nonlinearities, Arch. Math. 74 (2000), 129-146.

[8] P. Brenner, W. Von Wahl, Global classical solutions of nonlinear wave equations. Math. Z., 1981, 176: 87-121.

[9] A. Bulut, Maximizers for the Strichartz inequalities for the wave equation. Differential Integral Equations 23 (2010), 1035-1072.

[10] A. Bulut, Global well-posedness and scattering for the defocusing energy-supercritical cubic nonlinear wave equation. J. Func. Anal. 263 (2012), 1609-1660. MR2948225.

[11] A. Bulut, The radial defocusing energy-supercritical cubic nonlinear wave equation in $\mathbb{R}^{1+5}$. Nonlinearity, 27(2014), 1859-1877.

[12] A. Bulut, The defocusing energy-supercritical cubic nonlinear wave equation in dimension five. Trans. Amer. Math. Soc., 367(2015). 6017-6061.

[13] A. Bulut, The defocusing cubic nonlinear wave equation in the energy-supercritical regime. Contemporary Mathematics, 581, Amer. Math. Soc., Providence, RI, 2012.

[14] A. Bulut, M. Czubak, D. Li, N. Pavlovic and X. Zhang, Stability and unconditional uniqueness of solutions for energy critical wave equation in high dimensions. Comm. Partial Differential Equations, 38 (2013), 575-607.

[15] T. Cazenave, Uniform estimates for solutions of nonlinear Klein-Gordon equations. J. Funct. Anal. 60, 1 (1985), 36-55.

[16] T. Cazenave and P. L. Lions, Solutions globales d'équations de la chaleur semi linéaires. Comm. Partial Differential Equations 9, 10 (1984), 955-978.

[17] J.-Y. Chemin, About weak-strong uniqueness for the 3D incompressible Navier-Stokes system, Comm. Pure Appl. Math., 64(2011), 1587-1598.

[18] Q. Chen, C. Miao and Z. Zhang, On the uniqueness of weak solutions for the 3D NavierStokes equations, Ann. Inst. Henri Poincare-Nonlinear Analysis, 26(2009)2165-2180.

[19] M. Christ, J. Colliander, and T. Tao, Ill-posedness for nonlinear Schrödinger and wave equations, arXiv: 0311048

[20] M. Christ and M. Weinstein, Dispersion of small amplitude solutions of the generalized Korteweg-de Vries equation. J. Funct. Anal. 100 (1991), 87-109. MR1124294.

[21] D. Christodoulou and A. Tahvildar-Zadeh, On the asymptotic behavior of spherically symmetric wave maps. Duke Math. J. 71, 1 (1993), 31-69.

[22] C. Collot, Type II blow-up for the energy supercritical wave equation, 2014. arXiv: $1407.4525 \mathrm{v} 2$.

[23] R . Côte, C. Kenig, A. Lawrie, W. Schlag, Profiles for the radial focusing 4d energy-critical wave equation, arXiv:1402.2307. To appear in Comm. Math. Phys.

[24] R. Côte, and H. Zaag, Construction of a multi-soliton blow-up solution to the semilinear wave equation in one space dimension. Comm. Pure and Appl. Math., 66(2013), 1541-1581.

[25] W. Ding, On a conformally invariant elliptic equation on $\mathbb{R}^{n}$. Comm. Math. Phys. 107, 2 (1986), 331-335.

[26] B. Dodson, Global well-posedness and scattering for the radial, defocusing, cubic wave equation with almost sharp initial data, arXiv: 1604.04255.

[27] B. Dodson, Global well-posedness and scattering for the radial, defocusing, cubic wave equation with initial data in a critical Besove space, arXiv: 1608.02020.

[28] B. Dodson and A. Lawrie, Scattering for the radial 3d cubic wave equation. Analysis and PDE, 8(2015), 467-497.

[29] B. Dodson and A. Lawrie, Scattering for radial, semi-linear, super-critical wave equations with bounded critical norm. Arch. Rational Mech. Anal., 218(2015), 1459-1529.

[30] R. Donninger, H. Min, J. Krieger, W. Schlag, Exotic blowup solutions for the $u^{5}$ focusing wave equation in $\mathbb{R}^{3}$, Michigan Math. J., 63(2014), 451-501.

[31] R. Donninger and J. Krieger, Nonscattering solutions and blowup at infinity for the critical wave equation. Math. Anna., 357(2013), 89-163.

[32] R. Donninger and B. Schörkhuber. Stable self-similar blow up for energy subcritical wave equations. Dyn. Partial Differ. Equ., 9(1):63-87, 2012.

[33] R. Donninger and B. Schörkhuber, Stable blow up dynamics for energy supercritical wave equations. Trans. Amer. Math. Soc. 366, 4 (2014), 2167-2189.

[34] R. Donninger and B. Schörkhuber, On blowup in supercritical wave equations, 2014. Comm. Math. Phy., 346(2016), 907-943. 
[35] R. Donninger and B. Schörkhuber, Stable blowup for wave equations in odd space dimensions, To appear in Anna. de l'institut Henr. Poin. (C) Nonlinear Analysis.

[36] T. Duyckaerts, H. Jia, C.Kenig and F.Merle, Soliton resolution along a sequence of times for the focusing energy-critical wave equation, arXiv: 1601.01871.

[37] T. Duyckaerts, C.Kenig and F.Merle, Universality of blow-up profile for small radial type II blow-up solutions of the energy-critical equation. J. Eur. Math. Soc. 13(2011) 533-599.

[38] T. Duyckaerts, C. Kenig, F. Merle, Universality of the blow-up profile for small type II blow-up solutions of energy-critical wave equation, J. Eur. Math. Soc. (JEMS) 14:5 (2012), 1389-1454.

[39] T. Duyckaerts, C. Kenig, F. Merle, Classification of radial solutions of the focusing, energycritical wave equation, Cambridge Journal of Mathematics 1 (2013), no. 1, 75-144

[40] T. Duyckaerts, C. Kenig and F.Merle, Scattering for radial, bounded solutions of focusing supercritical wave equations. Int Math Res Notices IMRN, 2014, 224-258.

[41] T. Duyckaerts, C. Kenig, and F. Merle, Profiles of bounded radial solutions of the focusing, energy-critical wave equation. Geometric and Functional Analysis, 22(2012), 639-698.

[42] T. Duyckaerts, C. Kenig, F. Merle, Profiles for bounded solutions of dispersive equations, with applications to energy-critical wave and Schrödinger equations, Commun. Pure Appl. Anal. 14 (2015), no. 4, 1275-1326

[43] T. Duyckaerts and F. Merle, Dynamics of threshold solutions for energy-critical wave equation. Int. Math. Res. Pap. 2008, art. ID rpn002, 67 pp. MR 2416069.

[44] T. Duyckaerts, and F. Merle, Dynamic of threshold solutions for energy-critical NLS. Geom. Funct. Anal., 18(2009), 1787-1840.

[45] T. Duyckaerts, and F. Merle, Scattering norm estimate near the threshold for energy-critical focusing semilinear wave equation. Indiana Univ. Math. J., 58(2009), 1971-2001.

[46] T. Duyckaerts, and T. Roy, Blow-up of the critical Sobolev norm for nonscattering radial solutions of supercritical wave equations on $\mathbb{R}^{3}$,

[47] G. Furioli and E. Terraneo. Besov spaces and unconditional well-posedness for the nonlinear Schrödinger equation in $\dot{H}^{s}\left(\mathbb{R}^{n}\right)$. Commun. Contemp. Math. 5 (2003), no. 3, 349-367.

[48] G. Furioli, F. Planchon, E. Terraneo, Unconditional well-posedness for semilinear Schrödinger and wave equations in $H^{s}$. (English summary) Harmonic analysis at Mount Holyoke (South Hadley, MA, 2001), 147-156, Contemp. Math., 320, Amer. Math. Soc., Providence, RI, 2003.

[49] I. Gallagher and F. Planchon, On global solutions to a dofocusing semi-linear wave equation, Revista Mathematicá Iberoamericana, 19, 2003, pp. 161-177

[50] P. Germain, Strong solutions and weak-strong uniqueness for the nonhomogeneous NavierStokes system, J. Anal. Math., Vol. 105, 169-196, 2008.

[51] P. Germain, Weak-strong uniqueness for the isentropic compressible Navier-Stokes system, J. Math. Fluid Mech., Vol. 13, 137-146, 2011.

[52] P. Germain, Multipliers, paramultipliers, and weak-strong uniqueness for the Navier-Stokes equations, J. Differential Equations, Vol. 226, 373-428, 2006.

[53] B. Gidas, W. Ni and L. Nirenberg, Symmetry of positive solutions of nonlinear elliptic equatios in $\mathbb{R}^{n}$. In Mathematical analysis and applications, Part A, volume 7 of Adv. in Math. Suppl. Stud., pages 369-402. Academic Press, New York, 1981.

[54] Y. Giga and R. V. Kohn. Nondegeneracy of blowup for semilinear heat equations. Comm. Pure Appl. Math., 42(6):845-884, 1989.

[55] J. Ginibre and G. Velo, On the global Cauchy problem for nonlinear Klein-Gordon equation. Math. Z., 1985, 189: 487-505.

[56] J. Ginibre and G. Velo, On the global Cauchy problem for nonlinear Klein-Gordon equation II. Ann. Inst. Henri. Poincaré Analyse nonliéaire, 1989, 6: 15-35.

[57] J. Ginibre and G. Velo, Generalized Strichartz inequalities for the wave equation, J. Funct. Anal., 133(1995), 50-68.

[58] M. Grillakis, Regularity and asymptotic behaviour of the wave equation with a critical nonlinearity, Ann. of Math., 132(1990), 485-509.

[59] M. Grillakis, Regularity for nonlinear wave equation with critical nonlinearity, Comm. Pure Appl. Math., 1992, 45: 749-774.

[60] C. Gundlach, C. Critical phenomena in gravitational collapse. Living Rev. Relativ. 2 (1999), 1999-4, 58 pp. (electronic).

[61] M-A. Hamza, H. Zaag. Blow-up results for semilinear wave equations in the superconformal case. Discrete Contin. Dyn. Syst. Ser. B, 18(9):2315-2329, 2013. 
[62] M. Hillairet and P. Raphael, Smooth type II blow up solutions to the four dimensional energycritical wave equation, Analysis and PDE's, 5(2012), 777-829.

[63] L. Hörmander. The analysis of linear partial differential operators. III, volume 274 of Grundlehren der Mathematischen Wissenschaften [Fundamental Principles of Mathematical Sciences]. Springer-Verlag, Berlin, 1985. Pseudodifferential operators.

[64] S. Ibrahim, N. Masmoudi and K. Nakanishi, Scattering threshold for the focusing nonlinear Klein-Gordon equation, Analysis and PDE., 4 (2011), No3, 405-460.

[65] J. Jendrej. Construction of two-bubble solutions for energy-critical wave equations, arXiv: 1602.01093

[66] J. Jendrej. Nonexistence of radial two-bubbles with opposite signs for the energy-critical wave equation. Preprint, arXiv:1510.03965, 2015.

[67] J. Jendrej. Construction of type II blow-up solutions for the energy-critical wave equation in dimension 5. J. Funct. Anal., 272(2017), 866-917.

[68] J. Jendrej, Bounds on the speed of type II blow-up for the energy critical wave equation in the radial case, Int Math Res Notices (2016)2016(21): 6656-6688.

[69] H. Jia, C. Kenig, Asymptotic decomposition for semilinear wave and equivariant wave map equations, preprint 2015, arXiv 1503.06715

[70] K. Jörgen, Das Anfangswert problem im Grossen für eine nichlineare Wellengleichungen. Math. Z., 77(1961): 295-308.

[71] D. Joseph and T. Lundgren. Quasilinear Dirichlet problems deriven by positive sources. Arch. Rational Mech. Anal., 49: 241-269, 1972/1973.

[72] L. Kapitanski, Global and unique weak solution of nonlinear wave equations, Math. Res. Lett., 1(1994), 211-223.

[73] L. Kapitanski, Weak and Yet Weaker Solutions of Semilinear Wave Equations, Comm. Part. Diff. Eq., 19 (1994), 1629-1676.

[74] M. Keel and T. Tao, Endpoint Strichartz estimates. Amer. J. Math. 120:5(1998), 955-980.

[75] C. Kenig, The concentration-compactness/Rigidity method for critical dispersive and wave equations. https://www.math.uchicago.edu/ cek/Kenig.pdf.

[76] C. Kenig, Global well-posedness, scattering and blow up for the energy-critical, focusing, non-linear Schrödinger and wave equations. https://www.math.uchichago.edu/ cek/Kenigrev1.pdf.

[77] C. Kenig and F. Merle, Global well-posedness, scattering and blow-up for the energy critical focusing non-linear wave equation, Acta Math., 201 (2008), 147-212.

[78] C. Kenig and F. Merle, Scattering for $\dot{H}^{\frac{1}{2}}$ bounded solutions to the cubic, defocusing NLS in 3 dimensions. Trans. Amer. Math. Soc. 362 (2010), 1937-1962. MR2574882.

[79] C. Kenig and F.Merle, Nondispersive radial solutions to energy supercritical nonlinaer wave equations, with applications. Amer. J. Math. 133 (2011), no.4, 1029-1065.

[80] C. Kenig and F.Merle, Radial solutions to energy supercritical wave equations in odd dimensions. Disc. Cont. Dyn. Sys. A, 4 (2011) 1365-1381.

[81] C. Kenig, G. Ponce, and L. Vega. Well-posedness and scattering results for the generalized Korteweg-de Vries equation via the contraction principle. Comm. Pure Appl. Math., 46(4):527-620, 1993.

[82] C. E. Kenig, G. Ponce and L. Vega, Global well-posedness for semi-linear wave equations, Communications in partial differential equations 25 (2000), pp. 1741-1752

[83] R. Killip and M. Visan, The defocusing energy-supercritical nonlinear wave equation in three space dimensions, Trans. Amer. Math. Soc. 363 (2011), 3893-3934.

[84] R. Killip and M. Visan, The radial defocusing energy-supercritical nonlinear wave equation in all space dimensions. Proc. Amer. Math. Soc. 139 (2011), 1805-1817.

[85] J. Krieger, K. Nakanishi and W. Schlag, Global dynamics away from the ground state for the energy-critical nonlinear wave equation, Calculus of Variations and Partial Differential Equations, 44(2012), 1-45.

[86] J. Krieger and W. Schlag. On the focusing critical semi-linear wave equation. Amer. J. Math., 129(3):843-913, 2007.

[87] J. Krieger and W. Schlag. Full range of blow up exponents for the quintic wave equation in three dimensions. J. Math Pures Appl., 101(6):873-900, 2014.

[88] J. Krieger, W. Schlag and D. Tataru, Slow blow-up solutions for the $H^{1}\left(\mathbb{R}^{3}\right)$ critical focusing semilinear wave equation. Duke Math. J., 147(1): 1-53, 2009. 
[89] G. Lebeau, Optique non linéaire et ondes sur critiques, Séminaire: Équations aux Dérivées Partielles, 1999-2000, Exp. No. IV, 13 pp., Sémin. Équ. Dériv. Partielles, École Polytech., Palaiseau, 2000.

[90] J. Leray, Étude de diverses équations intégrales non linéaires et de quelques problèmes que pose l'hydrodynamique, J. Math. Pures Appl., IX. Sér. 12, 1-82, 1933.

[91] H. A. Levine, Instability and nonexistence of global solutions to nonlinear wave equations of the form $P u_{t t}=-A u+F(u) "$, Trans. Amer. Math. Soc. 192 (1974), 1-21.

[92] D. Li and X. Zhang, Dynamics for the energy critical nonlinear Schrödinger equation in high dimensions. J. Funct. Anal., 256(2009), 1928-1961.

[93] D. Li and X. Zhang, Dynamics for the energy critical nonlinear wave equation in high dimensions, Tran. AMS., 363 (2011) 1137-1160.

[94] H. Lindblad and C. Sogge, On existence and scattering with minimal regularity for semilinear wave equations, J. Funct. Anal., 130 (1995), 357-426.

[95] M. Majdoub, N. Masmoudi, On uniqueness for supercritical nonlinear wave and Schrödinger equations, Int Math Res Notices (2015) 2015(9): 2386-2405.

[96] N. Masmoudi and F. Planchon, On uniqueness for the critical wave equation. Comm. Partial Differential Equations 31 (2006), no. 7-9, 1099-1107.

[97] H. Matano and F. Merle, Classification of type I and type II behaviors for a supercritical nonlinear heat equation. J. Funct. Anal. 256, 4 (2009), 992-1064.

[98] H. Matano and F. Merle, Threshold and generic type I behaviors for a supercritical nonlinear heat equation. J. Funct. Anal. 261, 3 (2011), 716-748.

[99] R. May, Extension d'une classe d'unicité pour les équations de Navier-Stokes, Ann. Inst. H. Poincaré Anal. Non Linéaire, Vol. 27, 705-718, 2010.

[100] F. Merle, Lower bounds for the blowup rate of solutions of the Zakharov equation in dimension two. Comm. Pure Appl. Math. 49, 8 (1996), 765-794.

[101] F. Merle and P. Raphaël, Blow up of the critical norm for some radial $L^{2}$ super critical nonlinear Schrödinger equations. Amer. J. Math. 130, 4 (2008), 945-978.

[102] F. Merle, P. Raphaël, and J. Szeftel, The instability of Bourgain-Wang solutions for the $l^{2}$-critical NLS. Amer. Jour. Math., 135(2013), 967-1017.

[103] F. Merle and H. Zaag. Determination of the blow-up rate for the semilinear wave equation. Amer. J. Math., 125(5):1147-1164, 2003.

[104] F. Merle and H. Zaag. Determination of the blow-up rate for a critical semilinear wave equation. Math. Ann., 331(2):395-416, 2005.

[105] F. Merle, H.; Zaag. Openness of the set of non-characteristic points and regularity of the blow-up curve for the 1 D semilinear wave equation. Comm. Math. Phys., 282(1):55-86, 2008.

[106] F. Merle and H. Zaag. Dynamics near explicit stationary solutions in similarity variables for solutions of a semilinear wave equation in higher dimensions. Trans. Amer. Math. Soc., 368(2016), 27-87.

[107] F. Merle and H. Zaag. On the stability of the notion of non-characteristic point and blow-up profile for semilinear wave equations. Comm. Math. Phys., 333(2015), 1529-1562.

[108] C. Miao, Modern Methods to the Nonlinear Wave Equations, Monographs on Modern pure mathematics, No.133. Science Press, Beijing, 2010.4.

[109] C. Miao and B. Zhang, $H^{s}$-global well-posedness for semilinear wave equations, J. Math. Anal. Appl. 283(2003) 645-666.

[110] C. Miao, Y. Wu and X. Zhang, The defocusing energy-supercritical nonlinear wave equation in even space dimension. preprint.

[111] C. Miao, J. Zhang and J. Zheng, The defocusing energy-critical wave equation with a cubic convolution., Indiana University Math. Journal, 63(2014), 993-1015.

[112] Musso, M., and Pistoia, A. Sign changing solutions to a nonlinear elliptic problem involving the critical Sobolev exponent in pierced domains. J. Math. Pures Appl. (9) 86, 6 (2006), $510-528$.

[113] K. Nakanishi and W. Schlag, Invariant manifolds and dispersive Hailtonian evolution equations, Zürich Lectures in Advanced Mathematics, 2011.

[114] H. Pecher, $L^{p}$ Abschätzungen und klassische Lösungen fürnichr lineare wellengleichungen.I. Math. Z., 150(1976), 159-183.

[115] F. Planchon, On uniqueness for semilinear wave equations, Math. Z. 244 (2003), no. 3, $587-599$ 
[116] J. Rauch, The $u^{5}$-Klein-Gordon equation. Pitman Research Notes in Math. (Brezis H and Lions J L, eds), 1982, 53: 335-364.

[117] C. Rodriguez, Profiles for the radial focusing energy-critical wave equation in odd dimensions, Adv. Diff. Equa., 21(2016), 505-570.

[118] J. Ramos, A refinement of the Strichartz inequality for the wave equation with applications, Advances in Mathematics, 230(2012), 649-698.

[119] T. Roy, Global well-posedness for the radial defocusing cubic wave equation on $\mathbb{R}^{3}$ and for rough data, Elec. Jour. Diff. Equa., 2007(2007), 166: 1-22.

[120] T. Roy, Adapted Linear-Nonlinear Decomposition and Global well-posedness for solutions to the defocusing cubic wave equation on $\mathbb{R}^{3}$, Disc. Cont. Dynam. Systems A, 24(2009), $1307-1323$

[121] T. Roy, Global existence of smooth solutions of a 3D log-log energy-supercritical wave equation. Anal. PDE Vol. 2 (2009), 261-280.

[122] T. Roy, Scattering above energy norm of solutions of a loglog energy-supercritical Schrödinger equation with radial data. Journal of Differential Equations, 250 (2011), no. 1, 292-319.

[123] J. Shatah, M. Struwe, Regularity results for nonlinear wave equations, Ann. Math., 1993, 138: 505-518.

[124] J. Shatah, M. Struwe, Well posedness in the energy space for semilinear wave equation with critical growth, Inter. Math. Researth Notice, (1994), 303-309.

[125] J. Shatah and M. Struwe. Geometric wave equations, volume 2 of Courant Lecture Notes in Mathematics. New York University Courant Institute of Mathematical Sciences, New York, 1998.

[126] R. Shen, I-method for Defocusing, Energy-subcritical Nonlinear Wave Equation, arXiv: 1205.4739 .

[127] R. Shen, On the energy subcritical nonlinear wave equation in $\mathbb{R}^{3}$ with radial data. Analysis and PDE, 6 (2013), 1929-1987.

[128] Shih Hsi-Wei, Some results on scattering for log-subcritical and log-supercritical nonlinear wave equations, Anal. PDE Vol. 6 (2013), No. 1, 1-24.

[129] P. Sjölin, Global maximal estimates for solutions to the Schrödinger equation, Studia Math., 110(2) 1994, 105-114.

[130] A. Soffer. Soliton dynamics and scattering. In International Congress of Mathematicians. Vol. III, pages 459-471. Eur. Math. Soc., Zürich, 2006.

[131] C. D. Sogge, Lectures on Nonlinear Wave Equations, Monographs in Analysis II, International Press, 1995.

[132] W. A. Strauss, Nonlinear scattering theory at low energy, J. Funct. Anal., 41(1981), 110-133.

[133] W. A. Strauss, Nonlinear scattering theory at low energy sequel, J. Funct. Anal., 43(1981), 281-293.

[134] W. Strauss, Nonlinear wave equations, Conf. Board of the Math. Sciences, 73, Amer. Math. Soc., 1989.

[135] M. Struwe, Global regular solution to the $u^{5}$ Klein-Gordon equations. Ann Scu. Norm Sup. Pisa., 1988, 15: 495-513.

[136] M. Struwe. Uniqueness for critical nonlinear wave equations and wave maps via the energy inequality. Comm. Pure Appl. Math., 52(9): 1179-1188, 1999.

[137] M. Struwe, On uniqueness and stability for supercritical nonlinear wave and Schrödinger equations, Int. Math. Res. Not. 2006, Art. ID 76737, 14 pp.

[138] T. Tao, Low regularity semilinear wave equations, Comm. PDE. 24 (1999), 599-630.

[139] T. Tao, Spacetime bounds for the energy-critical nonlinear wave equation in three spatial dimensions, Dynamics of PDE, 3 (2006), 93-110.

[140] Tao T. Global regularity for a logarithmically supercritical defocusing nonlinear wave equation for spherically symmetric data. J. Hyperbolic Differ. Equ. 42 (2007), 259-265.

[141] G. Talenti, Best constant in Sobolev inequality. Ann. Mat. Pura Appl. (4), 110:353-372, 1976.

[142] N. Trudinger. Remarks concerning the conformal deformation of Riemannian structures on compact manifolds. Ann. Scuola Norm. Sup. Pisa (3), 22:265-274, 1968.

[143] L. Vega, Schrödinger equations: pointwise convergence to the initial data, Proceeding of the American Mathematical Society 102(1988), No.4, 874-878.

[144] W. Wahl, $L^{p}$ decay rates for the homogeneous wave equations. Math. Z., 1971, 120: 93-106. 
[145] B. G. Walther, Some $L^{p}\left(L^{\infty}\right)$ and $L^{2}\left(L^{2}\right)$-estimates for osciallatory Fourier transforms, in Analysis of Divergence (Orono, ME, 1997), Appl. Numer. Harmon. Anal., pp. 213-231, Birkhäuser, Boston, MA, 1999.

Institute of Applied Physics and Computational Mathematics, P. O. Box 8009, BeiJING, CHINA, 100088

E-mail address: miao_changxing@iapcm.ac.cn, zhengjiqiang@gmail.com 